\title{
Symplectic topology of Mañé's critical values
}

\author{
Kai CieliebaK \\ URS FRAUENFELDER \\ GABRIEL P PATERNAIN
}

\begin{abstract}
We study the dynamics and symplectic topology of energy hypersurfaces of mechanical Hamiltonians on twisted cotangent bundles. We pay particular attention to periodic orbits, displaceability, stability and the contact type property, and the changes that occur at the Mañé critical value $c$. Our main tool is Rabinowitz Floer homology. We show that it is defined for hypersurfaces that are either stable tame or virtually contact, and that it is invariant under homotopies in these classes. If the configuration space admits a metric of negative curvature, then Rabinowitz Floer homology does not vanish for energy levels $k>c$ and, as a consequence, these level sets are not displaceable. We provide a large class of examples in which Rabinowitz Floer homology is nonzero for energy levels $k>c$ but vanishes for $k<c$, so levels above and below $c$ cannot be connected by a stable tame homotopy. Moreover, we show that for strictly $1 / 4$-pinched negative curvature and nonexact magnetic fields all sufficiently high energy levels are nonstable, provided that the dimension of the base manifold is even and different from two.
\end{abstract}

53D40; 37D40

\section{Introduction}

In this paper we study the significance of Mañé's critical value to the symplectic topology of energy hypersurfaces. Steps in this direction were taken by Bernard [7], the third author, Polterovich and Siburg [62] and Viterbo [67], but here we will have a different focus. We will attempt to relate Mañé's critical value with stable Hamiltonian structures and a new type of Floer homology that we develop along the lines of the first two authors' paper [13], namely Rabinowitz Floer homology.

On the cotangent bundle $\tau: T^{*} M \rightarrow M$ of a closed manifold $M$ we consider autonomous Hamiltonian systems defined by a convex Hamiltonian

$$
H(q, p)=\frac{1}{2}|p|^{2}+U(q)
$$


and a twisted symplectic form

$$
\omega=d p \wedge d q+\tau^{*} \sigma
$$

Here $d p \wedge d q$ is the canonical symplectic form in canonical coordinates $(q, p)$ on $T^{*} M,|p|$ denotes the dual norm of a Riemannian metric $g$ on $M, U: M \rightarrow \mathbb{R}$ is a smooth potential, and $\sigma$ is a closed 2 -form on $M$. This Hamiltonian system describes the motion of a particle on $M$ subject to the conservative force $-\nabla U$ and the magnetic field $\sigma$.

We assume that $\sigma$ vanishes on $\pi_{2}(M)$, so its pullback $\pi^{*} \sigma$ to the universal cover $\pi: \widetilde{M} \rightarrow M$ is exact. The Mañé critical value ${ }^{1}$ is defined as

$$
c=c(g, \sigma, U):=\inf _{\theta} \sup _{q \in \widetilde{M}} \tilde{H}\left(q, \theta_{q}\right),
$$

where the infimum is taken over all 1 -forms $\theta$ on $\widetilde{M}$ with $d \theta=\pi^{*} \sigma$ and $\widetilde{H}$ is the lift of $H$.

We wish to understand how dynamical and symplectic topological properties of regular level sets $\Sigma_{k}=H^{-1}(k)$ change as $k$ passes the Mañé critical value. More specifically, we will investigate the following properties.

Dynamics The dynamics usually changes drastically at the Mañé critical value; we will provide abundant examples of this in Section 6 of this paper. We will pay particular attention to the existence or nonexistence of periodic orbits in given free homotopy classes of loops. The study of the existence of closed orbits of a charged particle in a magnetic field was initiated by V I Arnol'd [4] and S P Novikov [58] in the 1980's.

Displaceability A subset $A$ of a symplectic manifold $(V, \omega)$ is displaceable if there exists a Hamiltonian diffeomorphism (time-1 map of a time-dependent compactly supported Hamiltonian system) $\phi$ with $\phi(A) \cap A=\varnothing$. Among the many consequences of displaceability, the most relevant for this paper is the following result of Schlenk [64]: If for $H$ as above and some $k_{0}$ the set $\left\{H \leq k_{0}\right\}$ is displaceable, then it has finite Hofer-Zehnder capacity [40] and for almost every $k \leq k_{0}$ the energy level $\Sigma_{k}$ carries a contractible periodic orbit.

\footnotetext{
${ }^{1}$ More generally, there is a Mañé critical value associated to any cover of $M$ on which $\sigma$ becomes exact. In this paper, we will mostly restrict to the universal and abelian covers.
} 
Contact type A hypersurface $\Sigma$ in a symplectic manifold $\left(V^{2 n}, \omega\right)$ is of contact type if $\left.\omega\right|_{\Sigma}=d \lambda$ for a contact form $\lambda$ on $\Sigma$, ie a 1 -form such that $\lambda \wedge(d \lambda)^{n-1}$ is nowhere vanishing. This property was introduced by Weinstein [70] and has important dynamical consequences. For example, for large classes of contact type hypersurfaces the existence of a periodic orbit has been proved ("Weinstein conjecture"; see eg Hofer and Zehnder [40] and Viterbo [68]). Moreover, algebraic counts of periodic orbits can be organized into invariants of such hypersurfaces such as contact homology or symplectic field theory (see Eliashberg, Givental and Hofer [23]).

We say that a hypersurface $\Sigma$ is virtually contact if $\pi^{*} \omega \mid \widetilde{\Sigma}=d \lambda$ for a contact form $\lambda$ on a cover $\pi: \widetilde{\Sigma} \rightarrow \Sigma$ satisfying

$$
\sup _{x \in \widetilde{\Sigma}}\left|\lambda_{x}\right| \leq C<\infty, \quad \inf _{x \in \widetilde{\Sigma}} \lambda(R) \geq \varepsilon>0,
$$

where $|\cdot|$ is a metric on $\Sigma$ and $R$ is a vector field generating $\operatorname{ker}\left(\left.\omega\right|_{\Sigma}\right.$ ) (both pulled back to $\widetilde{\Sigma})$. A virtually contact homotopy is a smooth homotopy $\left(\Sigma_{t}, \lambda_{t}\right)$ of virtually contact hypersurfaces together with the corresponding 1 -forms on the covers such that the preceding conditions hold with constants $C, \varepsilon$ independent of $t$.

Stability and tameness A hypersurface $\Sigma$ in a symplectic manifold $\left(V^{2 n}, \omega\right)$ is stable if there exists a 1 -form $\lambda$ on $\Sigma$ which is nonzero on $\operatorname{ker}\left(\left.\omega\right|_{\Sigma}\right)$ and satisfies $\operatorname{ker}\left(\left.\omega\right|_{\Sigma}\right) \subset \operatorname{ker}(d \lambda)$. Note that contact type implies stability, but stability is more general, eg it allows for $\left.\omega\right|_{\Sigma}$ to be nonexact. The notion of stability was introduced by Hofer and Zehnder [40] and shares many consequences of the contact type condition, eg existence results for periodic orbits [40] and algebraic invariants arising from symplectic field theory [23]. A stable homotopy is a smooth homotopy $\left(\Sigma_{t}, \lambda_{t}\right)$ of stable hypersurfaces together with the corresponding 1-forms.

Suppose now $\omega$ vanishes on $\pi_{2}(V)$, and let $X(\Sigma)$ be the set of all closed characteristics in $\Sigma$ which are contractible in $V$. We define the function

$$
\Omega: X(\Sigma) \rightarrow \mathbb{R}
$$

by choosing for $v \in X(\Sigma)$ a filling disk $\bar{v}$ in $V$ which exists since $v$ is contractible in $V$, and setting

$$
\Omega(v)=\int \bar{v}^{*} \omega
$$

The pair $(\Sigma, \lambda)$ is said to be tame if there exists a positive constant $c$ such that

$$
\left|\int v^{*} \lambda\right| \leq c|\Omega(v)|
$$


for all $v \in X(\Sigma)$. Again, abundant examples of tame stable hypersurfaces will be given in Section 6, but we should mention that there are also examples of stable nontame hypersurfaces; see Contreras, Macarini and Paternain [21, Example 5.1] and Cieliebak and Volkov [17].

A stable homotopy $\left(\Sigma_{t}, \lambda_{t}\right)$ is said to be tame if each $\left(\Sigma_{t}, \lambda_{t}\right)$ is tame and the constant $c$ is independent of $t$.

We remark that when $V$ is a cotangent bundle $T^{*} M$, then $X(\Sigma)$ coincides with the closed orbits in $\Sigma$ whose projection to $M$ is contractible. Moreover, if $\operatorname{dim} M \geq 3$ and there is no potential, it follows from the homotopy sequence of a fibration that $\pi_{1}(\Sigma)$ injects into $\pi_{1}\left(T^{*} M\right)$ and thus $X(\Sigma)$ coincides with the set of closed orbits in $\Sigma$ which are contractible in $\Sigma$ itself.

Rabinowitz Floer homology In [13] the first two authors associated to a contact type hypersurface $\Sigma$ in a symplectic manifold $(V, \omega)$ (satisfying suitable conditions; see Section 4) its Rabinowitz Floer homology $\operatorname{RFH}(\Sigma)$. This is a graded $\mathbb{Z}_{2}$-vector space which is invariant under contact type homotopies of $\Sigma$. Its relevance for our purposes rests on the following two results in [13]: Displaceability of $\Sigma$ implies vanishing of $\operatorname{RFH}(\Sigma)$, and vanishing of $\operatorname{RFH}(\Sigma)$ implies the existence of a periodic orbit on $\Sigma$ which is contractible in $V$. To make these results applicable to the situation considered in this paper, we generalize in Section 4 the construction of Rabinowitz Floer homology to hypersurfaces that are stable tame or virtually contact, and to the corresponding homotopies. The generalization to the stable case is definitely nontrivial. The generalization to the virtually contact case is straightforward, but necessary, as we will try to explain in due time. In what follows we shall assume that $\Sigma$ is separating, ie $V \backslash \Sigma$ consists of two connected components.

We call a symplectic manifold $(V, \omega)$ geometrically bounded if it admits an $\omega$-compatible almost complex structure $J$ and a complete Riemannian metric such that

(i) there are positive constants $c_{1}$ and $c_{2}$ such that

$$
\omega(v, J v) \geq c_{1}|v|^{2}, \quad|\omega(u, v)| \leq c_{2}|u||v|
$$

for all tangent vectors $u$ and $v$ to $V$;

(ii) the sectional curvature of the metric is bounded above and its injectivity radius is bounded away from zero.

This is slightly stronger than the corresponding notion of Audin, Lalonde and Polterovich [6] since we demand $\omega$-compatibility. It is proved by Lu [46] that twisted cotangent bundles are geometrically bounded. 
Theorem 1.1 Let $(V, \omega)$ be a geometrically bounded symplectic manifold such that $\left.\omega\right|_{\pi_{2}(V)}=0$ (eg a twisted cotangent bundle as above).

(a) Rabinowitz Floer homology $\operatorname{RFH}(\Sigma)$ is defined for each tame stable hypersurface $\Sigma$ and invariant under tame stable homotopies.

(b) Rabinowitz Floer homology $\operatorname{RFH}(\Sigma)$ is defined for each virtually contact hypersurface $\Sigma$ and invariant under virtually contact homotopies provided that $\pi_{1}(\Sigma)$ injects into $\pi_{1}(V)$.

(c) If $\Sigma$ is as in (a) or (b) and is displaceable, then $\operatorname{RFH}(\Sigma)=0$.

(d) If $\Sigma$ is as in (a) or (b) satisfies $\operatorname{RFH}(\Sigma)=0$, then it carries a periodic orbit which is contractible in $V$.

We remark at once that Schlenk's result alluded to above can be derived right away from Theorem 1.1 if we assume that the hypersurface is tame. However, the compactness result proved in Section 4 will show that one can recover Schlenk's result fully, ie we can do without the tameness assumption, this is explained in Section 4.3.

One of the goals of this paper is to provide supporting evidence for the following paradigms:

$k>c$ : Above the Mañé critical value, $\Sigma_{k}$ is virtually contact. It may or may not be stable. Its Rabinowitz Floer homology $\operatorname{RFH}\left(\Sigma_{k}\right)$ is defined and nonzero, so $\Sigma_{k}$ is nondisplaceable. The dynamics on $\Sigma_{k}$ is like that of a geodesic flow; in particular, it has a periodic orbit in every nontrivial free homotopy class.

$k=c$ : At the Mañé critical value, $\Sigma_{k}$ is nondisplaceable and can be expected to be nonstable (hence noncontact). (When $M$ is the 2-torus, an example is given in [21] in which $\Sigma_{c}$ is of contact type.)

$k<c$ : Below the Mañé critical value, $\Sigma_{k}$ may or may not be of contact type. It is stable and displaceable (provided that $\chi(M)=0$ ), so its Rabinowitz Floer homology $\operatorname{RFH}\left(\Sigma_{k}\right)$ is defined and vanishes. In particular, $\Sigma_{k}$ has a contractible periodic orbit.

Some of these statements will be proved and others verified for various classes of examples, while some remain largely open. It should be said that the paradigms for energies $k \leq c$ are only rough approximations to the true picture. For example, work in progress of Macarini and the third author [48] shows that there are convex superlinear Hamiltonians on $\mathbb{T}^{n}$ (for any $n \geq 2$ ) for which $\Sigma_{c}$ is actually stable.

The first supporting evidence for the paradigms comes from the following:

Theorem 1.2 For $\sigma=0$ all the paradigms are true. Moreover, in this case all regular level sets are of contact type. 
A well-known example with $\sigma \neq 0$ where all the paradigms are true is a closed hyperbolic surface with $\sigma$ the area form; see Ginzburg [29]. Here for $k>c=1 / 2$, $\Sigma_{k}$ is contact and Anosov with all closed orbits noncontractible; for $k<1 / 2, \Sigma_{k}$ is stable (in fact contact with the opposite orientation) and the flow is completely periodic with contractible orbits; for $k=1 / 2, \Sigma_{k}$ is unstable and the flow is the horocycle flow without periodic orbits. We briefly discuss this example, as well as its generalization to higher dimensions, in Section 5.2.

Theorem 1.3 Suppose that $M$ admits a metric of negative curvature. Then for $k>c$ the Rabinowitz Floer homology $\operatorname{RFH}\left(\Sigma_{k}\right)$ is defined and does not vanish. In particular, $\Sigma_{k}$ is nondisplaceable for $k \geq c$.

Remark We point out that the chosen metric on $M$ need not have negative curvature (we merely ask the existence of such a metric on $M$ ) and the dynamics on $\Sigma_{k}$ need not be a small perturbation of the geodesic flow. The proof of Theorem 1.3 uses invariance of Rabinowitz Floer homology in a crucial way. In fact, existence of a metric of negative curvature is a technical hypothesis that can most likely be removed by establishing invariance of Rabinowitz Floer homology under more general deformations, eg deforming the symplectic form in a suitable class or adding an additional Hamiltonian term. ${ }^{2}$

If $\sigma$ is exact one can define another Mañé critical value

$$
c_{0}=c_{0}(g, \sigma, U):=\inf _{\theta} \sup _{q \in M} H\left(q, \theta_{q}\right),
$$

where the infimum is taken over all 1 -forms $\theta$ on $M$ with $d \theta=\sigma$. Note that $c_{0} \geq c$. For $k>c_{0}$ the level set $\Sigma_{k}$ encloses the Lagrangian graph $\operatorname{gr}(-\theta)$ and is therefore nondisplaceable by Gromov's theorem [32]. However, there are examples [61] with $c<c_{0}$. Nondisplaceability for the gap $c \leq k \leq c_{0}$ is new. In Theorem 5.5 we show that the gap appears rather frequently and in Section 6 we will explain how a gap of size $1 / 4$ appears quite explicitly in the geometry $\operatorname{PSL}(2, \mathbb{R})$.

We set $c_{0}:=\infty$ if $\sigma$ is nonexact. Then the contact type property behaves as follows with respect to the values $c$ and $c_{0}$. Suppose $\operatorname{dim} M \geq 3$. Then

- for $k>c_{0}, \Sigma_{k}$ is of contact type (Lemma 5.3);

- for $c<k \leq c_{0}, \Sigma_{k}$ is virtually contact but not of contact type (Lemma 5.1 and [18, Theorem B.1]).

${ }^{2}$ Note added in proof: Nonvanishing of $\operatorname{RFH}\left(\Sigma_{k}\right)$ has in the meantime been proven without the hypothesis of a metric of negative curvature, for $\sigma$ exact and $k>c_{0}$ in [1], and for general $\sigma$ and all $k>c$ in [55]. 
It turns out that whole intervals of unstable levels may appear in the gap. The first example where this phenomenon occurs was constructed in [61] on surfaces of negative curvature and with exact $\sigma$; see Theorem 5.4 below. With nonexact $\sigma$, this phenomenon always occurs for strictly 1/4-pinched negative sectional curvature.

Theorem 1.4 Let $(M, g)$ be a closed Riemannian manifold of even dimension different from two whose sectional curvature satisfies the pinching condition $-4 \leq K<-1$. Let $\sigma$ be a closed $2-$ form with cohomology class $[\sigma] \neq 0$. Then $c<c_{0}=\infty$ and for any $k>c$ sufficiently large, the hypersurface $\Sigma_{k}$ is not stable.

The theorem becomes false if the strict pinching condition is replaced by the weak one $-4 \leq K \leq-1$ : Compact quotients of complex hyperbolic space with $\sigma$ given by the Kähler form are stable for high energies (cf Section 5.2).

The next result is an easy consequence of the results by Schlenk and will be useful to show nonstability at the Mañé critical value in the various examples.

Theorem 1.5 Suppose that at the Mañé critical value $c, \Sigma_{c}$ has no contractible periodic orbits. Then $\Sigma_{c}$ is nonstable provided that all level sets $\Sigma_{k}$ with $k<c$ are displaceable.

More evidence for the displaceability of $\Sigma_{k}$ with $k<c$ comes from results of Laudenbach and Sikorav [45] and Polterovich [63] which assert that the zero section of $\left(T^{*} M, \omega\right)$ is actually displaceable if $\sigma$ is nonzero (assuming $\chi(M)=0$ ). Moreover, for a large class of cotangent bundles of solvable manifolds with $[\sigma] \neq 0$, displaceability for all $k<c=\infty$ is proved by Butler and the third author [10].

As an illustration of the homogeneous examples considered in Section 6, let $G$ be the 3-dimensional Heisenberg group of matrices

$$
\left(\begin{array}{lll}
1 & x & z \\
0 & 1 & y \\
0 & 0 & 1
\end{array}\right),
$$

where $x, y, z \in \mathbb{R}$. The 1 -form $\gamma:=d z-x d y$ is left-invariant and we let $\sigma:=d \gamma$ be the exact magnetic field. If $\Gamma$ is a co-compact lattice in $G, M:=\Gamma \backslash G$ is a closed 3-manifold and $\sigma$ descends to an exact 2-form on $M$. Now let $H$ be the left-invariant Hamiltonian defined in dual coordinates by

$$
2 H:=p_{x}^{2}+\left(p_{y}+x p_{z}\right)^{2}+p_{z}^{2} .
$$


Theorem 1.6 Consider $M, H$ and $\sigma$ as above. Then all the paradigms are true. More precisely: Each level $\Sigma_{k}$ except the Mañé critical level $c=c_{0}=1 / 2$ is stable and tame. For $k>1 / 2$ it is contact and $\operatorname{RFH}\left(\Sigma_{k}\right) \neq 0$. For $k<1 / 2$ it is noncontact and displaceable, so $\operatorname{RFH}\left(\Sigma_{k}\right)=0 . \Sigma_{k}$ has no contractible periodic orbits for $k \geq 1 / 2$. The level set $\Sigma_{1 / 2}$ is not stable.

By invariance of Rabinowitz Floer homology under tame stable homotopies, this implies:

Corollary 1.7 In the example of Theorem 1.6 two level sets $\Sigma_{k}, \Sigma_{\ell}$ with $k<1 / 2<\ell$ are smoothly homotopic and tame stable, but not tame stably homotopic.

An even more intriguing example arises on compact quotients $M:=\Gamma \backslash \operatorname{PSL}(2, \mathbb{R})$. Let $(x, y, \theta)$ be coordinates on $\operatorname{PSL}(2, \mathbb{R})$ arising from its identification with $S \mathbb{H}^{2} \cong$ $\mathbb{H}^{2} \times S^{1}$, the unit tangent bundle of the upper half plane. The left-invariant 1 -form $\gamma=d x / y+d \theta$ gives rise to an exact magnetic field $\sigma:=d \gamma$ on $M$. Let $H$ be the left-invariant Hamiltonian defined in dual coordinates by

$$
2 H:=\left(y p_{x}-p_{\theta}\right)^{2}+\left(y p_{y}\right)^{2}+p_{\theta}^{2} .
$$

Theorem 1.8 Consider $M, H$ and $\sigma$ as above. Then all the paradigms are true. More precisely: Each level $\Sigma_{k}$ except the Mañé critical levels $c=1 / 4$ and $c_{0}=1 / 2$ is stable and tame. For $k>1 / 2$ it is contact and $\operatorname{RFH}\left(\Sigma_{k}\right) \neq 0$. For $1 / 4<k<1 / 2$ it is noncontact and $\operatorname{RFH}\left(\Sigma_{k}\right) \neq 0$. For $k<1 / 4$ it is noncontact and displaceable, so $\operatorname{RFH}\left(\Sigma_{k}\right)=0 . \Sigma_{k}$ has no contractible periodic orbits for $k \geq 1 / 4$. The level sets $\Sigma_{1 / 4}$ and $\Sigma_{1 / 2}$ are not stable.

Remark Nonstability of the Mañé critical level $c$ in Theorems 1.6 and 1.8 follows from Theorem 1.5. Nonstability of the level $c_{0}$ in Theorem 1.8 follows from a detailed analysis of the dynamics on the level set. Note that the level $c_{0}$ is virtually contact; in fact for any $k>1 / 4, \Sigma_{k}$ is virtually contact.

Finally, we also point out the following examples with infinite Mañé critical value. An analogous picture arises on Sol-manifolds discussed in Section 6.4.

Theorem 1.9 Consider the $n$-torus $M=\mathbb{T}^{n}$ and a nonzero constant 2 -form $\sigma$. Then the Mañé critical value is $c=\infty$ and all level sets $\Sigma_{k}$ are noncontact, stable, tame and displaceable, so $\operatorname{RFH}\left(\Sigma_{k}\right)=0$ for all $k$.

We conclude this paper with a discussion of very high and low energy levels. For levels $k>c$ the only remaining issue is: 
Conjecture For $k>c$ the Rabinowitz Floer homology $\operatorname{RFH}\left(\Sigma_{k}\right)$ does not vanish. In particular, $\Sigma_{k}$ is nondisplaceable for $k \geq c$.

It is known that for $k>c, \Sigma_{k}$ carries a periodic orbit in every nontrivial free homotopy class of loops. (This is proved by the third author in [60] under a mild technical condition on $\pi_{1}(M)$, which is removed by Merry in [54].)

For $k<c$ the dynamics is much less well understood, even for very small values of $k$. If $\sigma \neq 0$, the results of Polterovich and Schlenk mentioned above yield a $k_{0}>0$ such that $\Sigma_{k}$ is displaceable for all $k \leq k_{0}$ and carries a contractible periodic orbit for almost all $k \leq k_{0}$. However, the following basic question is wide open:

Question Is $\Sigma_{k}$ stable for sufficiently small $k$, at least in the case that $\sigma$ is a symplectic form on $M$ ?

A positive answer to the question would give an alternative proof of the existence of closed contractible orbits on every low energy level for $\sigma$ symplectic. This has been recently proved by Ginzburg and Gürel [30]. In Section 6.5 (Proposition 6.19) we give an affirmative answer in the homogeneous symplectic case: Let $M=\Gamma \backslash G$ be a compact homogeneous space with a left-invariant metric and a left-invariant symplectic form $\sigma$. Then there exists $k_{0}>0$ such that for all $k<k_{0}$ the hypersurface $\Sigma_{k}$ is stable.

Acknowledgements We wish to thank L Macarini, F Schlenk and E Volkov for several useful discussions related to this paper. We also thank W Merry for comments and corrections on previous drafts. We thank the organizers of the wonderful Conference on Symplectic Geometry, Kyoto 2007, which inspired the present work. Finally, we thank the referee for numerous corrections. The first author was partially supported by DFG grant CI 45/1-3.

\section{Stable Hamiltonian structures and hypersurfaces}

Stable Hamiltonian structures Let $\Sigma$ be a closed oriented manifold of dimension $2 n-1$. A Hamiltonian structure on $\Sigma$ is a closed 2 -form $\omega$ such that $\omega^{n-1} \neq 0$. So its kernel $\operatorname{ker}(\omega)$ defines a 1-dimensional foliation which we call the characteristic foliation of $\omega$. We orient the characteristic foliation by a $1-$ form $\lambda$ such that $\lambda \wedge \omega^{n-1}>0$.

A Hamiltonian structure is called stable if there exists a $1-$ form $\lambda$ such that $\operatorname{ker} \omega \subset$ ker $d \lambda$ and $\lambda \wedge \omega^{n-1}>0$. We call $\lambda$ a stabilizing $1-$ form. Define the Reeb vector field $R$ by $\lambda(R)=1$ and $i_{R} \omega=0$ (which implies $i_{R} d \lambda=0$ ). 
The following two results give equivalent formulations of stability.

Theorem 2.1 (Wadsley [69]) A Hamiltonian structure $(\Sigma, \omega)$ is stable if and only if its characteristic foliation is geodesible, ie there exists a Riemannian metric such that all leaves are geodesics.

Theorem 2.2 (Sullivan [66]) A Hamiltonian structure $(\Sigma, \omega)$ is nonstable if and only if there exists a foliation cycle which can be arbitrarily well approximated by boundaries of singular 2-chains tangent to the foliation.

The simplest obstruction to stability that can appear in Sullivan's theorem is a Reeb component, ie an embedded annulus tangent to the characteristic foliation such that its boundary components are closed leaves with opposite orientations.

The following criterion for stability (whose proof is immediate) will be useful in later examples.

Lemma 2.3 Let $\left(\Sigma^{ \pm}, \omega^{ \pm}\right)$be stable Hamiltonian structures and $f: \Sigma^{+} \rightarrow \Sigma^{-}$a smooth (not necessarily injective) map which maps leaves diffeomorphically onto leaves. If a $1-$ form $\lambda$ stabilizes $\left(\Sigma^{-}, \omega^{-}\right)$, then $f^{*} \lambda$ stabilizes $\left(\Sigma^{+}, \omega^{+}\right)$.

For a Hamiltonian structure $(\Sigma, \omega)$ we denote by

$$
\Lambda(\Sigma, \omega):=\left\{\lambda \in \Omega^{1}(\Sigma): \operatorname{ker} \omega \subset \operatorname{ker} d \lambda, \lambda \wedge \omega^{n-1}>0\right\}
$$

the space of stabilizing 1-forms. It obviously satisfies:

Lemma 2.4 The space $\Lambda(\Sigma, \omega)$ is a convex cone in $\Omega^{1}(\Sigma)$. In particular, if it is nonempty, then it is contractible.

Stable hypersurfaces We call a closed oriented connected hypersurface $\Sigma$ in a connected symplectic manifold $\left(V^{2 n}, \omega\right)$ stable if the following holds:

(i) $\left.\omega\right|_{\Sigma}$ defines a stable Hamiltonian structure.

(ii) $\quad \Sigma$ is separating, ie $V \backslash \Sigma$ consists of two connected components.

Condition (a) in the following lemma gives a more dynamical formulation of stability and justification for its name. 
Lemma 2.5 [16] For a closed hypersurface $\Sigma$ in a symplectic manifold $(V, \omega)$ the following are equivalent:

(a) $\Sigma$ is stable in the sense of [40], ie there exists a tubular neighborhood $(-\varepsilon, \varepsilon) \times \Sigma$ of $\Sigma=\{0\} \times \Sigma$ such that the Hamiltonian line fields on $\{r\} \times \Sigma$ are conjugate for all $r \in(-\varepsilon, \varepsilon)$.

(b) There exists a vector field $Y$ transverse to $\Sigma$ such that $\operatorname{ker}\left(\left.\omega\right|_{\Sigma}\right) \subset \operatorname{ker}\left(\left.L_{Y} \omega\right|_{\Sigma}\right)$.

(c) $\left(\Sigma,\left.\omega\right|_{\Sigma}\right)$ is a stable Hamiltonian structure.

A stable homotopy of hypersurfaces in $(V, \omega)$ is a smooth homotopy $\left(\Sigma_{t}, \lambda_{t}\right)$ of stable hypersurfaces together with stabilizing 1 -forms.

Stable tubular neighbourhoods Now assume that $\Sigma$ is a stable hypersurface in a symplectic manifold $(V, \omega)$. We abbreviate

$$
\omega_{\Sigma}=\left.\omega\right|_{\Sigma}
$$

If $\lambda_{\Sigma} \in \Lambda\left(\Sigma, \omega_{\Sigma}\right)$ we call the pair $\left(\Sigma, \lambda_{\Sigma}\right)$ a stabilized hypersurface. For a stabilized hypersurface $\left(\Sigma, \lambda_{\Sigma}\right)$ a stable tubular neighbourhood is a pair $(\rho, \psi)$ where $\rho>0$ and $\psi:[-\rho, \rho] \times \Sigma \rightarrow V$ is an embedding satisfying

$$
\left.\psi\right|_{\{0\} \times \Sigma}=\left.\mathrm{id}\right|_{\Sigma}, \quad \psi^{*} \omega=\omega_{\Sigma}+d\left(r \lambda_{\Sigma}\right)=\omega_{\Sigma}+r d \lambda_{\Sigma}+d r \wedge \lambda_{\Sigma} .
$$

Note that a stable tubular neighbourhood satisfies condition (a) of Lemma 2.5. We abbreviate by $\mathcal{T}\left(\Sigma, \lambda_{\Sigma}\right)$ the space of stable tubular neighbourhoods of $\left(\Sigma, \lambda_{\Sigma}\right)$. We further denote by

$$
\mathcal{T}(\Sigma)=\bigcup_{\lambda_{\Sigma} \in \Lambda\left(\Sigma,\left.\omega\right|_{\Sigma}\right)} \mathcal{T}\left(\Sigma, \lambda_{\Sigma}\right)
$$

the space of stable tubular neighbourhoods for the stable (but not stabilized) hypersurface $\Sigma$. If $\left(\Sigma_{\sigma}, \lambda_{\sigma}\right)$ for $\sigma \in[0,1]$ is a stable homotopy of hypersurfaces we abbreviate by $\mathcal{T}\left(\left\{\Sigma_{\sigma}\right\}\right)$ the space of smooth families $\left(\rho_{\sigma}, \psi_{\sigma}\right)$ of stable tubular neighbourhoods.

Proposition 2.6 (a) Assume that $\left(\Sigma_{\sigma}, \lambda_{\sigma}\right)$ is a stable homotopy of compact hypersurfaces. Then $\mathcal{T}\left(\left\{\Sigma_{\sigma}\right\}\right)$ is nonempty.

(b) If $\Sigma$ is a compact stable hypersurface, $\mathcal{T}(\Sigma)$ is nonempty and path-connected.

Proof (a) Choose a smooth family of vector fields $X_{\sigma}$ on $V$ satisfying

$$
{ }^{\iota} X_{\sigma} \omega_{x}=\lambda_{\sigma}, \quad x \in \Sigma_{\sigma} .
$$

Since $\Sigma_{\sigma}$ is compact the flow $\phi_{X_{\sigma}}^{r}$ exists locally near $\Sigma_{\sigma}$. We observe that plugging in the Reeb vector field into (1) implies that $X_{\sigma}$ is transverse to $\Sigma_{\sigma}$. Hence we can 
define a smooth family of diffeomorphisms $\tilde{\psi}_{\sigma}:\left(-\tilde{\rho}_{\sigma}, \tilde{\rho}_{\sigma}\right) \times \Sigma_{\sigma} \rightarrow V$ for $\tilde{\rho}_{\sigma}>0$ by the formula

$$
\tilde{\psi}_{\sigma}(r, x)=\phi_{X_{\sigma}}^{r}(x), \quad(r, x) \in\left(-\tilde{\rho}_{\sigma}, \tilde{\rho}_{\sigma}\right) \times \Sigma_{\sigma} .
$$

We abbreviate

$$
\omega_{\sigma}=\omega_{\Sigma_{\sigma}}+d\left(r \lambda_{\sigma}\right) \text {. }
$$

Perhaps after shrinking $\tilde{\rho}_{\sigma}$ it follows that $\omega_{\sigma}$ is a symplectic structure on $\left(-\tilde{\rho}_{\sigma}, \tilde{\rho}_{\sigma}\right) \times \Sigma_{\sigma}$. Moreover, it follows from (1) that $\omega_{\sigma}$ and $\widetilde{\psi}_{\sigma}^{*} \omega$ agree at points of $\{0\} \times \Sigma_{\sigma}$. Applying Moser's argument ( see for example McDuff and Salamon [52, Lemma 3.14]), we find a smooth family of $\rho_{\sigma}>0$ and a smooth family of embeddings $\phi_{\sigma}:\left(-\rho_{\sigma}, \rho_{\sigma}\right) \times \Sigma_{\sigma} \rightarrow$ $\left(-\tilde{\rho}_{\sigma}, \tilde{\rho}_{\sigma}\right) \times \Sigma_{\sigma}$ satisfying

$$
\left.\phi_{\sigma}\right|_{\{0\} \times \Sigma_{\sigma}}=\mathrm{id}, \quad \phi_{\sigma}^{*} \widetilde{\psi}_{\sigma}^{*} \omega=\omega_{\sigma} .
$$

Now set

$$
\psi_{\sigma}=\tilde{\psi}_{\sigma} \circ \phi_{\sigma}
$$

Then $\left(\rho_{\sigma}, \psi_{\sigma}\right)$ lies in $\mathcal{T}\left(\left\{\Sigma_{\sigma}\right\}\right)$. In particular, $\mathcal{T}\left(\left\{\Sigma_{\sigma}\right\}\right)$ is nonempty.

(b) In view of part (a), $\mathcal{T}(\Sigma)$ is nonempty. To prove that it is path-connected we first show that for each $\lambda \in \Lambda\left(\Sigma, \omega_{\Sigma}\right)$ the space $\mathcal{T}(\Sigma, \lambda)$ is path-connected. Let $\left(\rho_{1}, \psi_{1}\right),\left(\rho_{2}, \psi_{2}\right) \in \mathcal{T}(\Sigma, \lambda)$. By hypothesis,

$$
\omega_{\lambda}=\left.\omega\right|_{\Sigma}+d(r \lambda)
$$

is symplectic on $U:=(-\rho, \rho)$ for some $\rho>\max \left(\rho_{1}, \rho_{2}\right)$. There exist neighbourhoods $U_{1}$ and $U_{2}$ of $\{0\} \times \Sigma$ in $U$ such that $\psi_{2}^{-1} \circ \psi_{1}: U_{1} \rightarrow U_{2}$ is an isomorphism and

$$
\left.\left(\psi_{2}^{-1} \circ \psi_{1}\right)^{*} \omega_{\lambda}\right|_{U_{2}}=\left.\omega_{\lambda}\right|_{U_{1}} \text {. }
$$

Note further that

$$
\left.\psi_{2}^{-1} \circ \psi_{1}\right|_{\{0\} \times \Sigma}=\mathrm{id} \text {. }
$$

Hence after choosing $U_{1}$ even smaller we can in a Weinstein neighbourhood identify the graph $\Gamma_{\psi_{2}^{-1} \circ \psi_{1}} \subset\left(U \times U, \omega_{\lambda} \oplus-\omega_{\lambda}\right)$ with an open subset of the graph $\Gamma_{\sigma} \subset T^{*} U$ of a closed one-form $\sigma$ on $U$. Considering the homotopy of graphs $\Gamma_{t \sigma}$ for $t \in[0,1]$ we find an $\epsilon>0$ and a path in $\mathcal{T}(\Sigma, \lambda)$ between $\left(\epsilon,\left.\psi_{1}\right|_{(-\epsilon, \epsilon) \times \Sigma}\right)$ and $\left(\epsilon,\left.\psi_{2}\right|_{(-\epsilon, \epsilon) \times \Sigma}\right)$. Now concatenating this path with shrinking paths between $\left(\rho_{1}, \psi_{1}\right)$ and $\left(\epsilon,\left.\psi_{1}\right|_{(-\epsilon, \epsilon) \times \Sigma)}\right.$ as well as between $\left(\rho_{2}, \psi_{2}\right)$ and $\left(\epsilon,\left.\psi_{2}\right|_{(-\epsilon, \epsilon) \times \Sigma}\right)$ we obtain a path between $\left(\rho_{1}, \psi_{1}\right)$ and $\left(\rho_{2}, \psi_{2}\right)$. This proves that for fixed $\lambda \in \Lambda\left(\Sigma, \omega_{\Sigma}\right)$ the space $\mathcal{T}(\Sigma, \lambda)$ is path-connected.

Now assume more generally that $\left(\rho_{0}, \psi_{0}\right),\left(\rho_{1}, \psi_{1}\right) \in \mathcal{T}(\Sigma)$. Then there exist $\lambda_{0}, \lambda_{1} \in$ $\Lambda\left(\Sigma, \omega_{\sigma}\right)$ such that

$$
\left(\rho_{0}, \psi_{0}\right) \in \mathcal{T}\left(\Sigma, \lambda_{0}\right), \quad\left(\rho_{1}, \psi_{1}\right) \in \mathcal{T}\left(\Sigma, \lambda_{1}\right)
$$


Since we have already seen that $\mathcal{T}\left(\Sigma, \lambda_{0}\right)$ and $\mathcal{T}\left(\Sigma, \lambda_{1}\right)$ are path-connected it suffices to connect arbitrary elements in these spaces by a path in $\mathcal{T}(\Sigma)$. To do that we make use of Lemma 2.4 giving us a path $\lambda_{\sigma}$ in $\Lambda\left(\Sigma, \omega_{\Sigma}\right)$ connecting $\lambda_{0}$ and $\lambda_{1}$. Hence we can apply part (a) to the stable homotopy $\left(\Sigma, \lambda_{\sigma}\right)$. This proves the proposition.

Contact structures A Hamiltonian structure $(\Sigma, \omega)$ is called contact if there exists a 1 -form $\lambda$ such that $d \lambda=\omega$ and $\lambda \wedge \omega^{n-1}>0$. In particular, $\lambda$ is a stabilizing 1 -form and $\lambda$ is a (positive) contact form, ie $\lambda \wedge(d \lambda)^{n-1}>0$. Note that $(\Sigma, \omega)$ can be contact only if $\omega$ is exact.

Sullivan's theory in [66] also provides a necessary and sufficient condition for an exact Hamiltonian structure $(\Sigma, \omega)$ being contact. Fix a positive vector field $R$ generating $\operatorname{ker} \omega$. Every Borel probability measure $\mu$ on $\Sigma$ invariant under the flow of $R$ gives rise to a 1 -current via

$$
\langle\mu, \beta\rangle=\int_{\Sigma} \beta(R) d \mu, \quad \beta \in \Omega^{1}(\Sigma) .
$$

We say $\mu$ is exact if it is exact as a 1 -current, ie $\langle\mu, \beta\rangle=0$ for all closed 1 -forms $\beta$.

Theorem 2.7 (McDuff [51]) An exact Hamiltonian structure $(\Sigma, \omega)$ is noncontact if and only if there exists a nontrivial exact positive invariant Borel measure $\mu$ such that $\langle\mu, \alpha\rangle=0$ for some (and hence every) 1 -form $\alpha$ with $d \alpha=\omega$.

The simplest obstruction to the contact property arises if $\mu$ is supported on a closed orbit: If there exists a null-homologous closed orbit $\gamma$ of $\operatorname{ker} \omega$ such that $\int_{\gamma} \alpha=0$ for a primitive $\alpha$ of $\omega$, then $(\Sigma, \omega)$ is noncontact.

The following immediate consequence of the theorem will be useful below.

Corollary 2.8 An exact Hamiltonian structure $(\Sigma, \omega)$ is noncontact provided there exist two nontrivial exact invariant Borel measures $\mu_{ \pm}$such that $\left\langle\mu_{+}, \alpha\right\rangle \geq 0$ and $\left\langle\mu_{-}, \alpha\right\rangle \leq 0$ for some (and hence every) $1-$ form $\alpha$ with $d \alpha=\omega$.

Proof For $\mu_{ \pm}$as in the corollary, some positive linear combination $\mu=a_{+} \mu_{+}+$ $a_{-} \mu_{-}$satisfies the condition in Theorem 2.7.

A particular invariant measure is given by the Liouville measure associated to a Hamiltonian structure $(\Sigma, \omega, R)$ with a chosen vector field $R$ generating $\operatorname{ker} \omega$. It is defined by the unique volume form $\mu \in \Omega^{2 n-1}(\Sigma)$ satisfying

$$
i_{R} \mu=\frac{\omega^{n-1}}{(n-1) !} \text {. }
$$


Lemma 2.9 Consider a twisted cotangent bundle $\left(T^{*} M, \omega=d p \wedge d q+\tau^{*} \sigma\right)$ with a convex Hamiltonian $H(q, p)=\frac{1}{2}|p|^{2}+U(q)$ as in the Introduction. If $M \neq \mathbb{T}^{2}$, then on every regular level set $\left(\Sigma_{k}=H^{-1}(k),\left.\omega\right|_{\Sigma_{k}}, R=X_{H}\right)$ the Liouville measure is exact as a current.

Proof We claim that $\left.\omega^{n-1}\right|_{\Sigma_{k}}$ is exact if $M \neq \mathbb{T}^{2}$. To see this, let $\theta:=p d q$ be the Liouville form and compute

$$
\omega^{n-1}=\left(d \theta+\tau^{*} \sigma\right)^{n-1}=(d \theta)^{n-1}+(n-1) \tau^{*} \sigma \wedge(d \theta)^{n-2} .
$$

On the right-hand side the first term is exact. For $n \geq 3$ the second term is exact as well and the claim follows, so it remains to consider the case $n=2$. If $k<\max U$ the projection $\tau_{k}:=\left.\tau\right|_{\Sigma_{k}}: \Sigma_{k} \rightarrow M$ is not surjective, so $\sigma$ is exact on the image of $\tau_{k}$ and the claim follows. If $k>\max U$ the Gysin sequence of the circle bundle $\tau_{k}: \Sigma_{k} \rightarrow M$ yields

$$
H^{0}(M ; \mathbb{R}) \stackrel{\cup e}{\longrightarrow} H^{2}(M ; \mathbb{R}) \stackrel{\tau_{k}^{*}}{\longrightarrow} H^{2}\left(\Sigma_{k} ; \mathbb{R}\right),
$$

where $e$ is the Euler class of the cotangent bundle of $M$. If $M \neq \mathbb{T}^{2}$ this Euler class is nonzero, so the first map is an isomorphism and $\tau_{k}^{*}$ the zero map. This proves the claim.

Now let $\Theta$ be a primitive of $\left.\omega^{n-1}\right|_{\Sigma_{k}}$ and $\beta \in \Omega^{1}\left(\Sigma_{k}\right)$ be closed. Then

$$
(n-1) ! \beta(R) \mu=(n-1) ! \beta \wedge i_{R} \mu=\left.\beta \wedge \omega^{n-1}\right|_{\Sigma_{k}}=\beta \wedge d \Theta=-d(\beta \wedge \Theta)
$$

is exact, so its integral over $\Sigma_{k}$ vanishes. This proves exactness of $\mu$ and hence the lemma.

The following immediate consequence of Lemma 2.9 will be used repeatedly in this paper.

Corollary 2.10 In the situation of Lemma 2.9, there exists no 1-form $\lambda$ on $\Sigma_{k}$ with $d \lambda=0$ and $\lambda(R)>0$.

\section{Tame hypersurfaces}

In this section we introduce the notion of weakly tame hypersurfaces in a symplectic manifold and the notion of tameness for stable hypersurfaces. As the name suggests, tame stable hypersurfaces are also weakly tame. We further explain what a tame, stable homotopy is. In the forthcoming Section 4 we then show how for weakly tame, stable 
hypersurfaces Rabinowitz Floer homology can be defined and that Rabinowitz Floer homology is invariant under tame, stable homotopies.

Given a closed hypersurface $\Sigma$ in a symplectically aspherical symplectic manifold $(V, \omega)$ we denote by $X(\Sigma)$ the set of closed characteristics in $\Sigma$ which are contractible in $V$. We define the function

$$
\Omega: X(\Sigma) \rightarrow \mathbb{R}
$$

by choosing for $v \in X(\Sigma)$ a filling disk $\bar{v}$ in $V$ which exists since $v$ is contractible in $V$ and putting

$$
\Omega(v)=\int \bar{v}^{*} \omega .
$$

Note that since $V$ is symplectically aspherical the function $\Omega$ is well-defined independent of the choice of the filling disk. We refer to the function $\Omega$ as the $\omega$-energy of a closed characteristic. For $a \leq b$ we abbreviate

$$
X_{a}^{b}(\Sigma)=\{v \in X(\Sigma): a \leq \Omega(v) \leq b\} .
$$

The hypersurface $\Sigma$ is called weakly tame if for each $a \leq b$ the space $X_{a}^{b}(\Sigma)$ is compact (with the topology of uniform convergence).

An example of weakly tame hypersurfaces are hypersurfaces of restricted contact type. Indeed if $\omega=d \lambda$ is exact and the restriction $\left.\lambda\right|_{\Sigma}$ is a contact form on $\Sigma$, then each closed characteristic can be parametrised as a periodic orbit of the Reeb vector field $R_{\lambda}$ and hence

$$
\Omega(v)=\int \bar{v}^{*} \omega=\int v^{*} \lambda=T_{\lambda}(v),
$$

where $T_{\lambda}(v)$ is the period of $v$ as a periodic orbit of the Reeb vector field $R_{\lambda}$. Therefore the theorem of Arzela-Ascoli implies that $\Sigma$ is weakly tame. On the other hand an obstruction for being weakly tame is the existence of a closed characteristic of vanishing $\omega$-energy. Indeed, taking iterates of it we get a sequence of closed characteristics of vanishing $\omega$-energy having no convergent subsequence.

If the hypersurface $\Sigma$ is stable we can define for each $\lambda \in \Lambda(\Sigma, \omega)$ another function

$$
T_{\lambda}: X(\Sigma) \rightarrow(0, \infty), \quad v \mapsto \int v^{*} \lambda
$$

The function $T_{\lambda}$ associates to $v$ its period as a Reeb orbit of the Reeb vector field $R_{\lambda}$. We note that for two different $\lambda_{1}, \lambda_{2} \in \Lambda(\Sigma, \omega)$ the functions $T_{\lambda_{1}}$ and $T_{\lambda_{2}}$ are proportional in the sense that there exist positive constants $\underline{c}_{\lambda_{1}, \lambda_{2}}$ and $\bar{c}_{\lambda_{1}, \lambda_{2}}$ such that

$$
\underline{c}_{\lambda_{1}, \lambda_{2}} T_{\lambda_{2}} \leq T_{\lambda_{1}} \leq \bar{c}_{\lambda_{1}, \lambda_{2}} T_{\lambda_{2}} .
$$


Indeed, the Reeb vector fields $R_{\lambda_{1}}$ and $R_{\lambda_{2}}$ are pointing in the same direction at each point of $\Sigma$ so that there exists a positive function $f_{\lambda_{1}, \lambda_{2}} \in C^{\infty}(\Sigma,(0, \infty))$ such that the formula

$$
R_{\lambda_{1}}=f_{\lambda_{1}, \lambda_{2}} R_{\lambda_{2}}
$$

holds. Since $\Sigma$ is compact the function $f_{\lambda_{1}, \lambda_{2}}$ attains a positive maximum and a positive minimum on $\Sigma$ and we set

$$
\underline{c}_{\lambda_{1}, \lambda_{2}}=\frac{1}{\max f_{\lambda_{1}, \lambda_{2}}}, \quad \bar{c}_{\lambda_{1}, \lambda_{2}}=\frac{1}{\min f_{\lambda_{1}, \lambda_{2}}}
$$

for which (3) holds.

A stable hypersurface is called tame if there exists $\lambda \in \Lambda(\Sigma, \omega)$ and a constant $c_{\lambda}$ such that for all $v \in X(\Sigma)$

$$
T_{\lambda}(v) \leq c_{\lambda}|\Omega(v)|
$$

We remark that it follows from (4) and the theorem of Arzela-Ascoli that a tame stable hypersurface is weakly tame. We refer to the constant $c_{\lambda}$ as a taming constant for $\lambda$. We note that if there exists a taming constant for one $\lambda_{1} \in \Lambda(\Sigma, \omega)$ then there exists also a taming constant for every other stabilizing one-form $\lambda_{2} \in \Lambda(\Sigma, \omega)$. Indeed, it follows from (3) that

$$
c_{\lambda_{2}}=\frac{c_{\lambda_{1}}}{\underline{c}_{\lambda_{1}, \lambda_{2}}}
$$

is a taming constant for $\lambda_{2}$. We finally introduce the tameness condition for stable homotopies.

A stable homotopy $\left(\Sigma_{t}, \lambda_{t}\right)$ for $t \in[0,1]$ is called tame, if there exists a taming constant $c>0$ such that

$$
T_{\lambda_{t}}(v) \leq c|\Omega(v)|, \quad \forall t \in[0,1], \forall v \in X\left(\Sigma_{t}\right)
$$

\section{Rabinowitz Floer homology}

Rabinowitz Floer homology as the Floer homology of Rabinowitz' action functional was, for the restricted contact type case, introduced by the first two authors in [13]. In this section we generalize as far as possible the construction of Rabinowitz Floer homology to the stable case. This generalization is not straightforward. The compactness proof for the moduli space of gradient flow lines has to be modified considerably in the stable case. The crucial point is the occurrence of a second Liapunov functional on the moduli space of gradient flow lines which is defined via a stabilizing 1 -form. One could define Rabinowitz Floer homology for a stabilized hypersurface by using a filtration from 
both action functionals. However, since the second action functional depends on the stabilizing form, such homology groups might depend on the stabilizing 1-form. To avoid this difficulty we only define Rabinowitz Floer homology in the case of weakly tame stable hypersurfaces, in which case this trouble does not occur.

As in the restricted contact type case the Rabinowitz Floer homology groups have the property that they vanish if the hypersurface is displaceable and that they coincide with the singular homology of the hypersurface in the case that there are no contractible Reeb orbits on the hypersurface. In particular, using Rabinowitz Floer homology one can recover a theorem due to Schlenk, that on displaceable stable hypersurfaces there always exists a contractible Reeb orbit. Schlenk's theorem actually does not need the weakly tame condition. And indeed, if one looks only at the local Rabinowitz Floer homology around the action value zero, the general compactness theorem for the moduli space of gradient flow lines of Rabinowitz action functional suffices, to recover Schlenk's theorem also in the not weakly tame case.

A further trouble in the stable case which one does not have to worry about in the restricted contact type case, is the difficulty that the stability condition is not open [15] and hence Rabinowitz action functional cannot be assumed to be generically Morse-Bott. We therefore have to introduce an additional perturbation which makes a generically perturbed Rabinowitz action functional Morse. It is however not clear that compactness for the moduli space of gradient flow lines for the perturbed Rabinowitz action functional continues to hold. Nevertheless, one can partially overcome this trouble be a procedure which is somehow reminiscent of the Conley index in the finite dimensional case. For a fixed action window it is possible to choose the perturbation so small that one can get a boundary operator by counting only gradient flow lines of the perturbed action functional which are sufficiently close to the gradient flow lines of the unperturbed action functional. Since the perturbation depends on the action window the drawback of this construction is, that one cannot define the full Rabinowitz Floer chain complex by using Novikov sums as in [13]. Instead one first defines filtered Rabinowitz Floer homology groups and then takes their inverse and direct limits. Because inverse and direct limits do not necessarily commute one obtains in this way actually two types of Rabinowitz Floer homology groups which we denote by $\overline{R F H}$ and $\underline{R F H}$. One can show [12] that in the restricted contact type case the Rabinowitz Floer homology $R F H$ as defined in [13] via Novikov sums coincides with $\overline{R F H}$. The two homology groups are connected by a canonical homomorphism

$$
\kappa: \overline{R F H} \rightarrow \underline{R F H} .
$$

It is an open question if $\kappa$ is always an isomorphism. The results of [12] suggest that it should at least be surjective. But even that is not clear yet, since in [12] the first two 
authors made heavy use of a bidirect system of chain complexes which we do not have in the stable case.

The Rabinowitz Floer homology groups are invariant under tame, stable homotopies. We prove this via an adiabatic version of Floer's continuation homomorphism. For this we need a compactness theorem for gradient flow lines in the case that Rabinowitz action functional is also allowed to depend on time.

This section is organised in the following manner. In Section 4.1 we recall the definition of Rabinowitz action functional, show how its critical points are given by Reeb orbits and derive the gradient flow equation.

In Section 4.2 the main ingredient for the compactness proof of the moduli spaces of gradient flow lines is established. Rabinowitz action functional is a Lagrange multiplier action functional and the main difficulty is to obtain a uniform bound on the Lagrange multiplier along gradient flow lines. Once this bound is established, the compactness for the moduli spaces of gradient flow lines follows from standard arguments well-known in Floer theory.

In Section 4.3 we give a new proof of Schlenk's theorem about the existence of a contractible Reeb orbit on a displaceable, stable hypersurface. This proof can also be used to derive the vanishing of Rabinowitz Floer homology for displaceable, weakly tame, stable hypersurfaces.

In Section 4.4 we introduce a class of perturbations of Rabinowitz action functional and show that for a generic perturbation Rabinowitz action functional becomes Morse. We then explain how for a fixed action window one can find small perturbations such that the moduli space of gradient flow lines can be written as the disjoint union of two closed parts where one part (which we refer to as the essential part) is compact. We then explain how the essential part can be used to define a boundary operator.

In Section 4.5 we define the two Rabinowitz Floer homology groups $\overline{R F H}$ and $\underline{R F H}$ for weakly tame, stable hypersurfaces. We show that both homology groups vanish for displaceable hypersurfaces and that both homology groups coincide with the singular homology of the hypersurface in the case that the hypersurface carries no contractible Reeb orbit.

In Section 4.6 we finally establish invariance of Rabinowitz Floer homology for tame, stable homotopies.

\subsection{Rabinowitz action functional}

We first give the general definition of Rabinowitz action functional and its gradient flow, postponing technical details to later subsections. 
Consider a symplectically aspherical manifold $(V, \omega)$ and a separating closed hypersurface $\Sigma \subset V$. Choose a Hamiltonian $\bar{H}$ with $\bar{H}^{-1}(0)=\Sigma$. Such Hamiltonians exist since $\Sigma$ is assumed to be separating. Denote by $\mathcal{L} \subset C^{\infty}\left(S^{1}, V\right)$ the component of contractible loops in the free loop space of $V$. Rabinowitz action functional

$$
\mathcal{A}^{\bar{H}}: \mathcal{L} \times \mathbb{R} \rightarrow \mathbb{R}
$$

is defined as

$$
\mathcal{A}^{\bar{H}}(v, \eta):=\int_{0}^{1} \bar{v}^{*} \omega-\eta \int_{0}^{1} \bar{H}(v(t)) d t, \quad(v, \eta) \in \mathcal{L} \times \mathbb{R},
$$

where $\bar{v}: D^{2} \rightarrow V$ is an extension of $v$ to the disk. One may think of $\mathcal{A}^{\bar{H}}$ as the Lagrange multiplier functional of the unperturbed action functional of classical mechanics also studied in Floer theory to a mean value constraint of the loop. The critical points of $\mathcal{A}^{\bar{H}}$ satisfy

$$
\left\{\begin{aligned}
\partial_{t} v(t) & =\eta X_{\bar{H}}(v(t)), \quad t \in \mathbb{R} / \mathbb{Z}, \\
\bar{H}(v(t)) & =0 .
\end{aligned}\right.
$$

Here we used the fact that $\bar{H}$ is invariant under its Hamiltonian flow.

It is also useful to consider Rabinowitz action functional for "weakly" time dependent Hamiltonians. Here weakly means that the Hamiltonian is just the product of a time independent Hamiltonian with a function depending only on time, ie

$$
H(t, y)=\chi(t) \bar{H}(y), \quad y \in V, t \in S^{1} .
$$

Normalizing, we assume in addition that

$$
\int_{0}^{1} \chi(t) d t=1
$$

We define Rabinowitz action functional $\mathcal{A}^{H}$ as before with $\bar{H}$ replaced by $H$. The critical point equation then becomes

$$
\left\{\begin{aligned}
\partial_{t} v(t) & =\eta \chi(t) X_{\bar{H}}(v(t)), \quad t \in \mathbb{R} / \mathbb{Z}, \\
\bar{H}(v(t)) & =0
\end{aligned}\right.
$$

ie the new critical points can just be obtained by reparametrisation of the previous ones. Moreover, the action value remains constant.

We next describe a class of metrics on $\mathcal{L} \times \mathbb{R}$. Pick a smooth $\omega$-compatible almost complex structure $J$ on $V$. For such a $J$ we define a metric $m$ on $\mathcal{L} \times \mathbb{R}$ for 
$(v, \eta) \in \mathcal{L} \times \mathbb{R}$ and $\left(\hat{v}_{1}, \hat{\eta}_{1}\right),\left(\hat{v}_{2}, \hat{\eta}_{2}\right) \in T_{(v, \eta)}(\mathcal{L} \times \mathbb{R})$ by

$$
m\left(\left(\widehat{v}_{1}, \hat{\eta}_{1}\right),\left(\widehat{v}_{2}, \hat{\eta}_{2}\right)\right)=\int_{0}^{1} \omega\left(\widehat{v}_{1}, J(v) \widehat{v}_{2}\right) d t+\hat{\eta}_{1} \cdot \hat{\eta}_{2} .
$$

The gradient of $\mathcal{A}^{H}$ with respect to this metric is given by

$$
\nabla \mathcal{A}^{H}=\nabla_{m} \mathcal{A}^{H}=\left(\begin{array}{c}
-J(v)\left(\partial_{t} v-\eta X_{H}(v)\right) \\
-\int_{0}^{1} H(t, v(\cdot, t)) d t
\end{array}\right) .
$$

Thus (positive) gradient flow lines of $\nabla \mathcal{A}^{H}$ are solutions $(v, \eta) \in C^{\infty}\left(\mathbb{R} \times S^{1}, V \times \mathbb{R}\right)$ of the following problem

$$
\left\{\begin{array}{r}
\partial_{s} v+J(v)\left(\partial_{t} v-\eta X_{H}(v)\right)=0, \\
\partial_{s} \eta+\int_{0}^{1} H(t, v(\cdot, t) d t=0 .
\end{array}\right.
$$

The boundary operator of Rabinowitz Floer homology counts gradient flow lines connecting critical points of $\mathcal{A}^{H}$. In order to prove that this is well defined one has to show that the moduli spaces of gradient flow line are compact modulo breaking. There are three difficulties one has to solve.

- An $L^{\infty}$-bound on the loop $v \in \mathcal{L}$.

- An $L^{\infty}$-bound on the Lagrange multiplier $\eta \in \mathbb{R}$.

- An $L^{\infty}$-bound on the derivatives of the loop $v$.

The first and the third point are standard problems in Floer theory one knows how to deal with: The $L^{\infty}$-bound for the loop follows from suitable assumptions on $(V, \omega)$ such as convexity at infinity or geometrical boundedness, and the derivatives can be controlled if $V$ is symplectically aspherical, meaning that $\omega$ vanishes on $\pi_{2}(V)$, and hence there is no bubbling of pseudo-holomorphic spheres.

The new feature is the bound on the Lagrange multiplier $\eta$, which can only hold under some additional hypothesis on the hypersurface $\Sigma$. It was derived in [13] for $\Sigma$ of restricted contact type. In the next subsection we will bound the Lagrange multiplier provided $\Sigma$ is stable.

\subsection{Bound on the Lagrange multiplier}

In this section we discuss the bound on the Lagrange multiplier. In the restricted contact case this was carried out in [13]. There the first two authors of this paper used the fact that in the restricted contact case the action value of Rabinowitz action functional at a 
critical point coincides with the Lagrange multiplier. This is not true anymore in the stable case. Nevertheless, using stability one can define a modified Rabinowitz action functional whose action value at critical points still is given by the Lagrange multiplier. However, the modified action is not necessarily a Liapunov function for gradient flow lines, so that the proof in [13] cannot be mimicked in the stable case by just using the modified version. The crucial observation is that the difference of the two action functionals is actually a Liapunov function for gradient flow lines and this fact can be used to still get a bound on the Lagrange multiplier.

In Section 4.2.1 we explain how the additional Liapunov functional arises and why it can be used to get the bound on the Lagrange multiplier. Technical details of Section 4.2.1 are postponed to Section 4.2.2. In Section 4.2.4 we also derive a bound on the Lagrange multiplier for gradient flow lines, when the Rabinowitz action functional itself is allowed to depend on time. For this we need a short stable homotopy. Using the notion of the stable pseudodistance, explained in Section 4.2.3, we can make precise what "short" means.

\subsubsection{A second Liapunov function for gradient flow lines From now on we assume} that $\Sigma \subset V$ is a stable hypersurface. We first explain the general strategy to obtain a bound on the Lagrange multiplier. Assume that $\lambda \in \Lambda(\Sigma, \omega)$ and let $R=R_{\lambda}$ be the Reeb vector field of the stabilizing 1 -form $\lambda$. We will use Hamiltonians $\bar{H}$ such that $\bar{H}^{-1}(0)=\Sigma$ and in addition $X_{\bar{H}}=R$ along $\Sigma$. To obtain the bound on the Lagrange multiplier we also have to choose $\bar{H}$ carefully outside the stable hypersurface. For that we have to assume that the stabilizing 1 -form $\lambda$ is small enough, but we will give a detailed explanation of this construction later in Section 4.2.2. The additional condition implies that the critical point equations (7) are equivalent to

$$
\left\{\begin{array}{clrl}
\partial_{t} v(t) & =\eta \chi(t) R(v(t)), & & t \in \mathbb{R} / \mathbb{Z}, \\
v(t) & \in \Sigma, & & t \in \mathbb{R} / \mathbb{Z},
\end{array}\right.
$$

ie $v$ is up to reparametrisation a periodic orbit of the Reeb vector field on $\Sigma$ with period $\eta .^{3}$

The bound on the Lagrange multiplier is derived by comparing the Rabinowitz action functional to a different action functional. This modified version of Rabinowitz action functional is obtained using an extension of the stabilizing 1 -form $\lambda$ on $\Sigma$ to a compactly supported 1 -form $\beta_{\lambda}$ on $V$. We also postpone the precise construction of

${ }^{3}$ The period $\eta$ may be negative or zero. We refer in this paper to Reeb orbits moved backwards as Reeb orbits with negative period and to constant orbits as Reeb orbits of period zero. 
$\beta_{\lambda}$. But given $\beta_{\lambda}$ we define the auxiliary action functional $\hat{\mathcal{A}}^{H}: \mathcal{L} \times \mathbb{R} \rightarrow \mathbb{R}$ by

$$
\hat{\mathcal{A}}^{H}(v, \eta):=\int_{0}^{1} \bar{v}^{*} d \beta_{\lambda}-\eta \int_{0}^{1} H(t, v(t)) d t,
$$

We will further use the difference of the two Rabinowitz action functionals

$$
\mathcal{A}:=\mathcal{A}^{H}-\hat{\mathcal{A}}^{H}: \mathcal{L} \times \mathbb{R} \rightarrow \mathbb{R}, \quad(v, \eta) \mapsto \int_{0}^{1} \bar{v}^{*}\left(\omega-d \beta_{\lambda}\right) .
$$

Note that $\mathcal{A}(v, \eta)$ is in fact independent of $\eta$. We further use an $\omega$-compatible almost complex structure $J$ on $V$ which has a special form on a stable tubular neighbourhood of $\Sigma$; see Section 4.2.2. For that $J$ we consider the following two bilinear forms on the tangent bundle $T(\mathcal{L} \times \mathbb{R})$ :

$$
\begin{aligned}
& m\left(\left(\widehat{v}_{1}, \hat{\eta}_{1}\right),\left(\hat{v}_{2}, \hat{\eta}_{2}\right)\right):=\int_{0}^{1} \omega\left(\widehat{v}_{1}, J \widehat{v}_{2}\right) d t+\hat{\eta}_{1} \hat{\eta}_{2}, \\
& \hat{m}\left(\left(\hat{v}_{1}, \hat{\eta}_{1}\right),\left(\hat{v}_{2}, \hat{\eta}_{2}\right)\right):=\int_{0}^{1} d \beta_{\lambda}\left(\widehat{v}_{1}, J \hat{v}_{2}\right) d t+\hat{\eta}_{1} \hat{\eta}_{2} .
\end{aligned}
$$

Here the bilinear form $m$ (which has already been defined in the previous subsection) is positive definite. The main point in the choice of $H, \beta_{\lambda}$ and $J$ is to make sure that the following Proposition becomes true.

Proposition 4.1 If $(v, \eta) \in \mathcal{L} \times \mathbb{R}$ and $(\hat{v}, \hat{\eta}) \in T_{(v, \eta)}(\mathcal{L} \times \mathbb{R})$ then the following two assertions hold:

(i) $d \hat{\mathcal{A}}^{H}(v, \eta)(\hat{v}, \hat{\eta})=\hat{m}\left(\nabla_{m} \mathcal{A}^{H}(v, \eta),(\widehat{v}, \hat{\eta})\right)$,

(ii) $(m-\hat{m})((\hat{v}, \hat{\eta}),(\hat{v}, \hat{\eta})) \geq 0$.

We prove Proposition 4.1 in Section 4.2.2. Assertion (i) might be interpreted as

$$
\nabla_{\widehat{m}} \hat{\mathcal{A}}^{H}=\nabla_{m} \mathcal{A}^{H} .
$$

However, we point out that the gradient with respect to the bilinear form $\hat{m}$ is not uniquely determined since $\hat{m}$ is not positive definite. We can use Proposition 4.1 to show that the difference action functional $\mathcal{A}$ is a Liapunov function for the gradient flow lines of $\mathcal{A}^{H}$.

Corollary 4.2 The functional $\mathcal{A}$ is nondecreasing along gradient flow lines of Rabinowitz action functional. 
Proof Let $w \in C^{\infty}(\mathbb{R}, \mathcal{L} \times \mathbb{R})$ be a gradient flow line of Rabinowitz action functional, ie, a solution of

$$
\partial_{s} w(s)=\nabla_{m} \mathcal{A}^{H}(w(s)), \quad s \in \mathbb{R} .
$$

We estimate using Proposition 4.1

$$
\begin{aligned}
\frac{d}{d s} \mathcal{A}(w) & =d \mathcal{A}^{H}(w)\left(\partial_{s} w\right)-d \hat{\mathcal{A}}^{H}(w)\left(\partial_{s} w\right) \\
& =m\left(\nabla_{m} \mathcal{A}^{H}(w), \nabla_{m} \mathcal{A}^{H}(w)\right)-\hat{m}\left(\nabla_{m} \mathcal{A}^{H}(w), \nabla_{m} \mathcal{A}^{H}(w)\right) \\
& \geq 0 .
\end{aligned}
$$

This concludes the proof of the Corollary.

Since the restriction of $\beta_{\lambda}$ to $\Sigma$ equals $\lambda$ we obtain using Stokes' theorem and (9) the following period-action equality for critical points $(v, \eta)$ of $\mathcal{A}^{H}$,

$$
\hat{\mathcal{A}}^{H}(v, \eta)=\eta \text {. }
$$

An elaboration of this observation is the second assertion of the following Lemma which might be thought of as a period-action inequality for almost Reeb orbits. For the statement of the Lemma we use the abbreviation

$$
\|H\|_{\infty}=\int_{0}^{1} \sup _{V}|H| d t=\|\bar{H}\|_{\infty} .
$$

Lemma 4.3 If the stabilizing 1 -form $\lambda$ is small enough in the $C^{0}$-topology, then for every $\epsilon>0$ and for every $\gamma \in[0,1)$ we can choose the Hamiltonian $\bar{H}$ in such a way that for every $\chi$ satisfying (6) the following two conditions hold for $H=\chi \bar{H}$.

(i) $\|H\|_{\infty} \leq \gamma+\epsilon$.

(ii) For $(v, \eta) \in \mathcal{L} \times \mathbb{R}$ the following implication holds:

$$
\left\|\nabla \mathcal{A}^{H}(v, \eta)\right\| \leq \frac{2 \gamma}{3} \Rightarrow|\eta| \leq \frac{1}{1-\gamma}\left|\hat{\mathcal{A}}^{H}(v, \eta)\right|+\frac{2 \gamma}{3(1-\gamma)} .
$$

Here the norms and the gradient are taken with respect to $m$.

Proof The Lemma follows from Lemma 4.7 below.

Proposition 4.4 Suppose that the stabilizing $1-$ form $\lambda$ is small enough and $H$ is as in Lemma 4.3 for $\epsilon>0$ and $\gamma \in(0,1-\epsilon]$. Assume that $w=(v, \eta) \in C^{\infty}(\mathbb{R}, \mathcal{L} \times \mathbb{R})$ is a gradient flow line of $\nabla \mathcal{A}^{H}$ for which there exist $a \leq b$ such that

$$
\mathcal{A}^{H}(w)(s), \mathcal{A}(w)(s) \in[a, b], \quad \forall s \in \mathbb{R} .
$$


Then the $L^{\infty}$-norm of $\eta$ is bounded by

$$
\|\eta\|_{\infty} \leq\left(\frac{9-5 \gamma}{4 \gamma(1-\gamma)}\right)(b-a)+\frac{2 \gamma}{3(1-\gamma)}+\frac{9(b-a)}{4 \gamma^{2}} \epsilon .
$$

Proof For $\sigma \in \mathbb{R}$ we set

$$
\tau(\sigma)=\inf \left\{\tau \geq 0:\left\|\nabla \mathcal{A}^{H}(w(\sigma+\tau))\right\| \leq \frac{2 \gamma}{3}\right\} .
$$

To obtain a bound on $\tau(\sigma)$ we estimate using the gradient flow equation and (10)

$$
\begin{aligned}
b-a & \geq \mathcal{A}^{H}(w(\sigma))-\mathcal{A}^{H}(w(\sigma+\tau(\sigma)) \\
& =\int_{\sigma}^{\sigma+\tau(\sigma)} \frac{d}{d s}\left(\mathcal{A}^{H}(w)\right) d s \\
& =\int_{\sigma}^{\sigma+\tau(\sigma)} d \mathcal{A}^{H}(w)\left(\partial_{s} w\right) d s \\
& =\int_{\sigma}^{\sigma+\tau(\sigma)}\left\|\nabla \mathcal{A}^{H}(w)\right\|^{2} d s \\
& \geq\left(\frac{2 \gamma}{3}\right)^{2} \tau(\sigma)
\end{aligned}
$$

from which we deduce

$$
\tau(\sigma) \leq \frac{9(b-a)}{4 \gamma^{2}}
$$

Furthermore, for every $s \in \mathbb{R}$ the modified version of Rabinowitz action functional can be estimated from above using (10) again by

$$
\left|\hat{\mathcal{A}}^{H}(w(s))\right|=\left|\mathcal{A}^{H}(w(s))-\mathcal{A}(w(s))\right| \leq b-a .
$$

The above two inequalities together with Lemma 4.3 and the equation $\partial_{s} \eta(s)=$ $-\int_{0}^{1} H(t, v(s, t)) d t$ following from the gradient flow equation imply

$$
\begin{aligned}
|\eta(\sigma)| & \leq|\eta(\sigma+\tau(\sigma))|+\int_{\sigma}^{\sigma+\tau(\sigma)}\left|\partial_{s} \eta(s)\right| d s \\
& \leq \frac{1}{1-\gamma}(b-a)+\frac{2 \gamma}{3(1-\gamma)}+(\gamma+\epsilon) \tau(\sigma) \\
& \leq\left(\frac{9-5 \gamma}{4 \gamma(1-\gamma)}\right)(b-a)+\frac{2 \gamma}{3(1-\gamma)}+\frac{9(b-a)}{4 \gamma^{2}} \epsilon .
\end{aligned}
$$

Since $\sigma \in \mathbb{R}$ was arbitrary the proposition follows. 
Corollary 4.5 Suppose that the stabilizing 1 -form is small enough. Then for given $a \leq b$ the Hamiltonian $\bar{H}=\bar{H}(b-a)$ can be chosen in such a way that for every $\chi$ satisfying (6) and for every gradient flow line $w=(v, \eta) \in C^{\infty}(\mathbb{R}, \mathcal{L} \times \mathbb{R})$ of $\nabla \mathcal{A}^{H}$ which satisfies (10) the $L^{\infty}$-norm of $\eta$ is bounded by

$$
\|\eta\|_{\infty} \leq\left(\frac{5}{2}\right)^{2}(b-a)+2 .
$$

Proof Choose $\gamma=3 / 5$ and $\epsilon \leq 4 /(25(b-a))$ and let $\bar{H}=\bar{H}(b-a)$ be the corresponding Hamiltonian. With these choices the Corollary follows from Proposition 4.4.

Remark The Corollary is somehow the optimal Corollary from Proposition 4.4, since the function

$$
f \in C^{\infty}((0,1), \mathbb{R}), \quad \gamma \mapsto \frac{9-5 \gamma}{4 \gamma(1-\gamma)}
$$

attains at the point $\gamma=3 / 5$ its unique minimum.

4.2.2 Admissible tuples In this section we prove Proposition 4.1 and Lemma 4.3.

For $\lambda \in \Lambda(\Sigma, \omega)$ denote by $\mathcal{I}(\Sigma, \lambda)$ the space of $\omega_{\Sigma}$-compatible complex structures on the bundle $\xi_{\lambda}=\operatorname{ker} \lambda$. Note that actually $\mathcal{I}(\Sigma, \lambda)$ only depends on the ray of $\lambda \in \Lambda(\Sigma, \omega)$ since $\xi_{\sigma \lambda}=\xi_{\lambda}$ for $\sigma>0$. For $I \in \mathcal{I}(\Sigma, \lambda)$ we introduce the quantity

$$
\kappa(\lambda, I):=\sup _{\substack{w \in \xi_{\lambda} \\ w \neq 0}} \frac{|d \lambda(w, I w)|}{\omega(w, I w)} \geq 0 .
$$

We define the upper semicontinuous function

$$
\kappa: \Lambda(\Sigma, \omega) \rightarrow[0, \infty), \quad \lambda \mapsto \inf _{I \in \mathcal{I}(\Sigma, \lambda)} \kappa(\lambda, I) .
$$

Note that $\kappa$ has the following scaling behaviour

$$
\kappa(\sigma \lambda)=\sigma \kappa(\lambda), \quad \sigma>0, \lambda \in \Lambda(\Sigma, \omega) .
$$

Recall from Section 2 the notion of a stable tubular neighbourhood. We further introduce the lower semicontinuous function

$$
\varrho: \Lambda(\Sigma, \omega) \rightarrow(0, \infty)
$$

measuring the size of stable tubular neighbourhoods which is defined for $\lambda \in \Lambda(\Sigma, \omega)$ by

$$
\varrho(\lambda)=\sup \{\rho:(\rho, \psi) \in \mathcal{T}(\Sigma, \lambda)\}
$$


The scaling behaviour for the function $\varrho$ is given by

$$
\varrho(\sigma \lambda)=\frac{1}{\sigma} \varrho(\lambda)
$$

Definition A stabilizing 1-form $\lambda \in \Lambda(\Sigma, \omega)$ is called small if

$$
\max \left\{\kappa(\lambda), \frac{1}{\varrho(\lambda)}\right\}<1
$$

We abbreviate by $\Lambda_{s}(\Sigma, \omega) \subset \Lambda(\Sigma, \omega)$ the subset of small stabilizing 1 -forms.

Remark It follows from the scaling behaviour of the functions $\kappa$ and $\varrho$ that for every $\lambda \in \Lambda(\Sigma, \omega)$ there exists $\tau>0$ such that $\tau \lambda$ is small.

We introduce three spaces of functions. Given $\rho>1$ the first space

$$
\mathcal{F}_{1}(\rho) \subset C^{\infty}(\mathbb{R}, \mathbb{R})
$$

consists of functions $\phi$ for which there exists $\rho_{0} \in(1, \rho)$ such that the following conditions are met:

$$
\left\{\begin{aligned}
\phi(r) & =r+1, & & r \in\left[-\rho_{0}, \rho_{0}\right], \\
\phi^{\prime}(r) & \leq 1, & & r \in \mathbb{R}, \\
\operatorname{supp}(\phi) & \subset(-\rho, \rho) . & &
\end{aligned}\right.
$$

Given $\gamma \geq 0$ and $\epsilon>0$ the second space

$$
\mathcal{F}_{2}(\gamma, \epsilon) \subset C^{\infty}(\mathbb{R}, \mathbb{R})
$$

consists of functions $h$ for which the following holds:

$$
\left\{\begin{aligned}
h(r) & =r, & & r \in[-\gamma, \gamma], \\
0 \leq h^{\prime}(r) & \leq 1+\epsilon, & & r \in \mathbb{R}, \\
h(r) & =\gamma+\epsilon & & r \geq \gamma+\epsilon, \\
h(r) & =-\gamma-\epsilon & & r \leq-\gamma-\epsilon .
\end{aligned}\right.
$$

The third space

$$
\mathcal{F}_{3} \subset C^{\infty}\left(S^{1}, \mathbb{R}\right)
$$

consists of functions $\chi \in C^{\infty}\left(S^{1}, \mathbb{R}\right)$ satisfying

$$
\int_{0}^{1} \chi(t) d t=1
$$

Remark All three spaces $\mathcal{F}_{1}(\rho), \mathcal{F}_{2}(\gamma, \epsilon)$ and $\mathcal{F}_{3}$ are convex and nonempty. 
Definition A stable tubular neighbourhood $\tau=(\rho, \psi) \in \mathcal{T}(\Sigma, \lambda)$ is called large if $\rho>1$. We abbreviate by $\mathcal{T}_{\ell}(\Sigma, \lambda) \subset \mathcal{T}(\Sigma, \lambda)$ the subset of large stable tubular neighbourhoods.

We remark that if $\lambda$ is a small stabilizing 1 -form, then the space of large tubular neighbourhoods is not empty by definition of small.

Definition Given $\epsilon>0$ and $\gamma \in[0,1-\epsilon]$ a $(\gamma, \epsilon)$-admissible quintuple for the stable hypersurface $\Sigma$ in $V$

$$
\alpha=(\lambda, \tau, \phi, h, \chi)
$$

consists of $\lambda \in \Lambda_{s}(\Sigma, \omega), \tau=(\rho, \psi) \in \mathcal{T}_{\ell}(\Sigma, \lambda), \phi \in \mathcal{F}_{1}(\rho), h \in \mathcal{F}_{2}(\gamma, \epsilon)$, and $\chi \in \mathcal{F}_{3}$.

Remark We sometimes omit the reference to $\gamma$ and $\epsilon$ and refer to a $(\gamma, \epsilon)$-admissible quintuple as an admissible quintuple.

We abbreviate by

$$
\mathfrak{A}^{\gamma, \epsilon}=\mathfrak{A}^{\gamma, \epsilon}(\Sigma, V)
$$

the space of all $(\gamma, \epsilon)$-admissible quintuples for $\Sigma$ in $V$. We denote by

$$
\pi: \mathfrak{A}^{\gamma, \epsilon} \rightarrow \Lambda_{S}(\Sigma, \omega)
$$

the projection to the first factor. Note that for each $\lambda \in \Lambda_{s}(\Sigma, \omega)$ the fiber

$$
\mathfrak{A}_{\lambda}^{\gamma, \epsilon}=\pi^{-1}(\lambda)
$$

is nonempty. As we have seen in Corollary 4.5 and the Remark following it the somehow optimal choice to bound the Lagrange multiplier is $\gamma=3 / 5$. Hence for $\epsilon \in(0,2 / 5]$ we set

$$
\mathfrak{A}^{\epsilon}=\mathfrak{A}^{3 / 5, \epsilon} \text {. }
$$

We further abbreviate

$$
\mathfrak{A}=\bigcup_{\epsilon \in(0,2 / 5]} \mathfrak{A}^{\epsilon}
$$

For a stable tubular neighbourhood $\tau=(\rho, \psi) \in \mathcal{T}(\Sigma, \lambda)$ we denote by

$$
U_{\tau}=\psi([-\rho, \rho] \times \Sigma)
$$

its image in $V$. We introduce a compactly supported 1-form on $V$ for an admissible quintuple which extends the $1-$ form $\lambda$ on $\Sigma$ by

$$
\beta_{\alpha}(y)= \begin{cases}\phi(r) \lambda(x) & y=\psi(x, r) \in U_{\tau}, \\ 0 & y \in V \backslash U_{\tau} .\end{cases}
$$


We will also sometimes suppress some of the dependencies of $\beta_{\alpha}$ and write

$$
\beta=\beta_{\lambda}=\beta_{\alpha} .
$$

Using the notion of admissible quintuples we are now in position to give the precise definition of the Rabinowitz action functional. For a $(\gamma, \epsilon)$-admissible quintuple $\alpha=(\lambda, \tau, \phi, h, \chi)$ with $\tau=(\rho, \psi)$ we define $H \in C^{\infty}\left(V \times S^{1}, \mathbb{R}\right)$ for $y \in V$ by the condition

$$
H(y, t)=H_{\alpha}(y, t)= \begin{cases}\chi(t) h(r) & y=\psi(x, r) \in U_{\tau}, t \in S^{1}, \\ H(y, t)= \pm \chi(t)(\gamma+\epsilon) & y \in V \backslash U_{\tau}, t \in S^{1} .\end{cases}
$$

For later reference we introduce the abbreviation

$$
\bar{H}(y)=\int_{0}^{1} H(t, y) .
$$

We sometimes use the notation

$$
\mathcal{A}^{\alpha}=\mathcal{A}^{H}, \quad \hat{\mathcal{A}}^{\alpha}=\hat{\mathcal{A}}^{H}, \quad \mathcal{A}_{\alpha}=\mathcal{A}
$$

to make the dependency of the three action functionals on the parameters explicit.

To define the bilinear form $m$ and $\hat{m}$ we have to pick a suitable subspace of $\omega-$ compatible almost complex structures on $V$. We first make the following definition.

Definition If $\lambda \in \Lambda(\Sigma, \omega)$ then the space of small $\omega_{\Sigma}$-compatible almost complex structures on $\xi_{\lambda}$ is defined as

$$
\mathcal{I}_{S}(\Sigma, \lambda)=\{I \in \mathcal{I}(\Sigma, \lambda): \kappa(\lambda, I) \leq 1\} .
$$

We note that if $\lambda$ is small, then by definition the space $\mathcal{I}_{S}(\Sigma, \lambda)$ is nonempty.

Recall from the introduction that a compatible almost complex structure $J$ is called geometrically bounded if $\omega(\cdot, J \cdot)$ is a complete Riemannian metric with bounded sectional curvature and injectivity radius bounded away from zero.

If $\alpha$ is an admissible quintuple, and $I \in \mathcal{I}_{S}(\Sigma, \pi(\alpha))$ we denote by $\mathcal{J}(\alpha, I)$ the subspace of $\omega$-compatible geometrically bounded almost complex structures $J$ on $V$ which split on the stable tubular neighbourhood $U_{\tau}=\psi([-\rho, \rho] \times \Sigma) \subset V$ with respect to the decomposition

$$
\begin{gathered}
\left.T V\right|_{U_{\tau}}=\xi_{\lambda} \oplus \xi_{\lambda}^{\omega} \\
J=\left(\begin{array}{ll}
I & 0 \\
0 & J_{0}
\end{array}\right)
\end{gathered}
$$


where $J_{0}$ is the standard complex structure on the symplectic complement of $\xi_{\lambda}$ spanned by the Reeb vector field $R_{\lambda}$ and $\partial / \partial r$. We further set

$$
\mathcal{J}(\alpha)=\bigcup_{I \in \mathcal{I}_{S}(\Sigma, \pi(\alpha))} \mathcal{J}(\alpha, I) .
$$

The following Lemma is the main technical point to establish Proposition 4.1.

Lemma 4.6 Assume that $\alpha=(\lambda, \tau, \phi, h, \chi)$ is an admissible quintuple for the stable hypersurface $\Sigma$ in $V$, and $J \in \mathcal{J}(\alpha)$. Then for every $w \in T V$ the following estimate holds:

$$
d \beta_{\alpha}(w, J w) \leq \omega(w, J w) .
$$

Proof The inequality (13) is clear on the complement of the stable tubular neighbourhood $U_{\tau}$ since there $d \beta_{\alpha}$ vanishes and $\omega(\cdot, J \cdot)$ is positive definite. For $\tau=(\rho, \psi)$ we identify via $\psi$ the neighbourhood $U_{\tau}$ of $\Sigma$ in $V$ symplectically with $[-\rho, \rho] \times \Sigma$ and $\omega=\omega_{\Sigma}+d(r \lambda)$. Then the exterior derivative of $\beta_{\alpha}$ is given by the formula

$$
\left.d \beta_{\alpha}\right|_{U_{\tau}}=\phi(r) d \lambda+\phi^{\prime}(r) d r \wedge \lambda .
$$

For $u=(r, x) \in U_{\tau}$ and $w \in T_{u} V$ we write $w=w_{1}+w_{2}$ with respect to the decomposition $\left.T V\right|_{U_{\tau}}=\xi_{\lambda} \oplus \xi_{\lambda}^{\omega}$. Note that since $J \in \mathcal{J}(\alpha)$ there exists $I \in \mathcal{I}_{S}(\Sigma, \lambda)$ such that

$$
J w=I w_{1}+J_{0} w_{2} .
$$

We first observe that the following implications follow from (11)

$$
\phi(r) \geq 0 \Rightarrow \phi(r) \leq r+1, \quad \phi(r) \leq 0 \Rightarrow \phi(r) \geq r+1 .
$$

Moreover, since $I \in \mathcal{I}_{s}(\Sigma, \lambda)$ it follows that

$$
\left|d \lambda\left(w_{1}, I w_{1}\right)\right| \leq \omega_{\Sigma}\left(w_{1}, I w_{1}\right) .
$$

Using further $\phi^{\prime} \leq 1$ following from (11) we estimate

$$
\begin{aligned}
d \beta_{\alpha}(w, J w) & =\phi(r) d \lambda\left(w_{1}, I w_{1}\right)+\phi^{\prime}(r) d r \wedge \lambda\left(w_{2}, J_{0} w_{2}\right) \\
& \leq \max \left\{(r+1) d \lambda\left(w_{1}, I w_{1}\right), 0\right\}+\omega\left(w_{2}, J w_{2}\right) \\
& =\max \left\{r d \lambda\left(w_{1}, I w_{1}\right)+\omega_{\Sigma}\left(w_{1}, I w_{1}\right), 0\right\}+\omega\left(w_{2}, J w_{2}\right) \\
& =\omega\left(w_{1}, J w_{1}\right)+\omega\left(w_{2}, J w_{2}\right) \\
& =\omega(w, J w) .
\end{aligned}
$$

This proves the Lemma. 
Proof of Proposition 4.1 The inequality (13) implies that

$$
(m-\hat{m})((\hat{v}, \hat{\eta}),(\hat{v}, \hat{\eta}))=(m-\hat{m})((\widehat{v}, 0),(\widehat{v}, 0)) \geq 0 .
$$

Recall that the gradient of $\mathcal{A}^{H}$ with respect to the metric $m$, defined by the equation

$$
d \mathcal{A}^{H}(v, \eta)(\hat{v}, \hat{\eta})=m\left(\nabla_{m} \mathcal{A}^{H}(v, \eta),(\hat{v}, \hat{\eta})\right),
$$

is given by $\quad \nabla_{m} \mathcal{A}^{H}(v, \eta)=\left(-J(v)\left(\dot{v}-\eta X_{H}(v)\right), \int_{0}^{1} H(v) d t\right)$.

Here the Hamiltonian vector field $X_{H}$, defined by the equation $d H=-i_{X_{H}} \omega$, equals zero outside the region $[-\rho, \rho] \times \Sigma$ and on this region it is given by $X_{H}=\chi(t) h^{\prime}(r) R$. Hence the stability condition $i_{R} d \lambda=0$ together with the first equality in (11) yields $d H=-i_{X_{H}} d \beta_{\alpha}$, which in turn implies that

$$
d \hat{\mathcal{A}}^{H}(v, \eta)(\hat{v}, \hat{\eta})=\hat{m}\left(\nabla_{m} \mathcal{A}^{H}(v, \eta),(\hat{v}, \hat{\eta})\right) .
$$

This proves the Proposition.

We finally show Lemma 4.3 by proving the following Lemma whose proof is an elaboration of the proof of [13, Proposition 3.1]. To see how this Lemma actually implies Lemma 4.3 it only remains to note if $\alpha$ is a $(\gamma, \epsilon)$-admissible quintuple, then

$$
\left\|H_{\alpha}\right\|_{\infty}=\gamma+\epsilon \text {. }
$$

Lemma 4.7 Assume that $\alpha=(\lambda, \tau, \phi, h, \chi)$ is a $(\gamma, \epsilon)$-admissible quintuple with $\tau=(\rho, \psi)$. Then the following implication holds:

$$
\left\|\nabla \mathcal{A}^{\alpha}(v, \eta)\right\| \leq \frac{2 \gamma}{3} \Rightarrow|\eta| \leq \frac{1}{1-\gamma}\left(\left|\hat{\mathcal{A}}^{\alpha}(v, \eta)\right|+\left\|\nabla \mathcal{A}^{\alpha}(v, \eta)\right\|\right) .
$$

Proof For $\rho^{\prime} \leq \rho$ we use the following notation for the stable tubular subneighbourhood of $\tau$ :

$$
\tau_{\rho^{\prime}}=\left(\rho^{\prime},\left.\psi\right|_{\left[-\rho^{\prime}, \rho^{\prime}\right] \times \Sigma}\right) .
$$

The Lemma is proved in two steps.

Step 1 Assume that $v(t)$ for every $t \in S^{1}$ is contained in the stable tubular neighbourhood $U_{\tau_{\gamma}}$. Then the inequality for $|\eta|$ holds.

We first note that the Hamiltonian vector field and the one-form satisfy on $U_{\tau_{\gamma}}$ the relation

$$
\left.\beta_{\alpha}\left(X_{H}\right)\right|_{S^{1} \times U_{\tau_{\gamma}}}=\left.H\right|_{S^{1} \times U_{\tau_{\gamma}}}+\chi
$$


In the following estimate we denote by \|\|$_{1}$ and \|\|$_{2}$ the $L^{1}-$ and, respectively, $L^{2}$-norm on the circle.

$$
\begin{aligned}
\left|\widehat{\mathcal{A}}^{\alpha}(v, \eta)\right| & =\left|\int_{0}^{1} v^{*} \beta_{\alpha}-\eta \int_{0}^{1} H(t, v(t)) d t\right| \\
& =\left|\eta \int_{0}^{1} \beta_{\alpha}\left(X_{H}(v)\right) d t+\int_{0}^{1} \beta_{\alpha}\left(\partial_{t} v-\eta X_{H}(v)\right) d t-\eta \int_{0}^{1} H(t, v(t)) d t\right| \\
& \geq\left|\eta \int_{0}^{1} \chi d t\right|-\left|\int_{0}^{1} \beta_{\alpha}\left(\partial_{t} v-\eta X_{H}(v)\right) d t\right| \\
& \geq|\eta|-(1+\gamma)\left\|\partial_{t} v-\eta X_{H}(v)\right\|_{1} \\
& \geq|\eta|-(1+\gamma)\left\|\partial_{t} v-\eta X_{H}(v)\right\|_{2} \\
& \geq|\eta|-(1+\gamma)\left\|\nabla \mathcal{A}^{\alpha}(v, \eta)\right\| .
\end{aligned}
$$

This proves Step 1.

Step 2 Assume that $\left\|\nabla \mathcal{A}^{\alpha}(v, \eta)\right\| \leq 2 \gamma / 3$. Then $v(t) \in U_{\tau_{\gamma}}$ for every $t \in S^{1}$.

We argue by contradiction and exclude the following two cases.

Case 1 There exists $t_{0}, t_{1} \in S^{1}$ such that $v\left(t_{0}\right) \in U_{\tau_{2 \gamma / 3}}$ and $v\left(t_{1}\right) \in V \backslash U_{\tau_{\gamma}}$.

Case 2 For all $t \in S^{1}$, we have that $v(t) \in V \backslash U_{\tau_{\gamma}}$.

We first observe that in Case 1 there exist two disjoint intervals $I^{1}=\left[s_{0}^{1}, s_{1}^{1}\right] \subset S^{1}$ and $I^{2}=\left[s_{0}^{2}, s_{1}^{2}\right]$ such that

$$
v\left(s_{0}^{1}\right) \in \partial U_{\tau_{\gamma}}, \quad v\left(s_{1}^{1}\right) \in \partial U_{\tau_{2 \gamma / 3}}, \quad v\left(s_{0}^{2}\right) \in \partial U_{\tau_{2 \gamma / 3}}, \quad v\left(s_{1}^{2}\right) \in \partial U_{\tau_{\gamma}},
$$

and

$$
v(s) \in U_{\tau_{\gamma}} \backslash U_{\tau_{2 \gamma / 3}}, s \in I^{1} \cup I^{2} .
$$

Identifying $U_{\tau_{\gamma}}$ with $[-\gamma, \gamma] \times \Sigma$ we write for $s \in I^{1} \cup I^{2}$

$$
v(s)=(r(s), u(s)) \in[-\gamma, \gamma] \times \Sigma .
$$

We estimate

$$
\begin{aligned}
\left\|\nabla \mathcal{A}^{\alpha}(v, \eta)\right\| & >\left\|\partial_{t} v-\eta \chi X_{H}(v)\right\|_{2} \\
& \geq\left\|\partial_{t} v-\eta \chi X_{H}(v)\right\|_{1} \\
& \geq \int_{s_{0}^{1}}^{s_{1}^{1}}\left|\partial_{t} r(s)\right| d s+\int_{s_{0}^{2}}^{s_{1}^{2}}\left|\partial_{t} r(s)\right| d s \\
& \geq \frac{2 \gamma}{3} .
\end{aligned}
$$

This contradiction excludes Case 1 . 
To exclude Case 2 we estimate

$$
\left\|\nabla \mathcal{A}^{\alpha}\right\| \geq\left|\int_{0}^{1} H(t, v(t)) d t\right|>\frac{2 \gamma}{3},
$$

which contradicts the assumption. Thus Step 2 and hence the Lemma follow.

4.2.3 The stable pseudo-distance We later prove invariance of Rabinowitz Floer homology via an adiabatic homotopy argument. For that we need short homotopies of stable hypersurfaces. In order to say what "short" means we introduce the stable pseudo-distance.

We define the stable pseudo-distance as the infimum of the length of paths of stable quadruples between two stable hypersurfaces. To begin with here is the definition of a stable quadruple.

Definition A stable quadruple in $V$

$$
\mathfrak{S}=(\Sigma, \lambda, \tau, I)
$$

consists of a stable hypersurface $\Sigma \subset V$, a small stabilizing 1 -form $\lambda \in \Lambda_{s}(\Sigma, \omega)$, a large tubular neighbourhood $\tau \in \mathcal{T}_{\ell}(\Sigma, \lambda)$ and a small $\omega_{\Sigma}$-compatible almost complex structure $I \in \mathcal{I}_{s}(\Sigma, \lambda)$. We also refer to a stable quadruple as above as a stable quadruple for the stable hypersurface $\Sigma$.

Let $\mathfrak{P}=\left\{\mathfrak{S}_{\zeta}\right\}=\left\{\left(\Sigma_{\zeta}, \lambda_{\zeta}, \tau_{\zeta}, I_{\zeta}\right)\right\}$ for $\zeta \in[0,1]$ be a smooth path of stable quadruples. To be precise, we require for a smooth path $\tau_{\zeta}$ of large stable tubular neighbourhoods that for a fixed $\rho>1$ we have a smooth family of maps $\psi_{\zeta}:[-\rho, \rho] \times \Sigma \rightarrow V$ where $\Sigma$ is a fixed manifold diffeomorphic to every $\Sigma_{\zeta} \subset V$ such that $\tau_{\zeta}=\left(\rho, \psi_{\zeta}\right) \in \mathcal{T}_{\ell}\left(\Sigma_{\zeta}, \lambda_{\zeta}\right)$ for every $\zeta \in[0,1]$. We associate to such a path a distance in the following way. For fixed $\zeta \in[0,1]$ define a vector field on $[-\rho, \rho] \times \Sigma$ by

$$
X_{\psi_{\zeta}}(r, x)=\left.\psi_{\zeta}^{*} \frac{d}{d \theta}\right|_{\theta=0} \psi_{\zeta+\theta}(r, x), \quad(r, x) \in[-\rho, \rho] \times \Sigma .
$$

We refer to the expression

$\mathfrak{V}_{\mathfrak{P}}(\zeta)=\rho\left(\max _{[-\rho, \rho] \times \Sigma_{\zeta}}\left|\omega\left(X_{\psi \zeta}, R_{\lambda_{\zeta}}\right)\right|+\max _{[-\rho, \rho] \times \Sigma_{\zeta}}\left|d \iota_{X_{\psi_{\zeta}}} \lambda_{\zeta}\left(R_{\lambda_{\zeta}}\right)\right|+\max _{\Sigma_{\zeta}}\left|\dot{\lambda}_{\zeta}\left(R_{\lambda_{\zeta}}\right)\right|\right)$

as the speed of the path $\mathfrak{P}$ at $\zeta$. We define the length of the path $\mathfrak{P}$ by the formula

$$
\Delta(\mathfrak{P})=\int_{0}^{1} \mathfrak{V}_{\mathfrak{P}}(\zeta) d \zeta
$$


The stable pseudo-distance between two stable hypersurfaces $\Sigma_{0}$ and $\Sigma_{1}$

$$
\Delta\left(\Sigma_{0}, \Sigma_{1}\right) \in[0, \infty]
$$

is defined as the infimum of the length of all paths of stable quadruples whose endpoints are stable quadruples for the stable hypersurfaces $\Sigma_{0}$ respectively $\Sigma_{1}$. Here we understand that the stable pseudo-distance is infinite if there is no such path.

4.2.4 The time-dependent case In this section we establish a bound on the Lagrange multiplier for gradient flow lines, when the Rabinowitz action functional is allowed to depend itself on time.

Given two stable hypersurfaces $\Sigma^{-}, \Sigma^{+} \subset V$ and $\alpha^{ \pm} \in \mathfrak{A}\left(\Sigma^{ \pm}, V\right)$ we denote by

$$
\mathcal{H}\left(\alpha^{-}, \alpha^{+}\right) \subset C^{\infty}(V \times \mathbb{R})
$$

the space of time dependent Hamiltonians $H \in C^{\infty}(V \times \mathbb{R})$ for which there exists $R>0$ with the property that the family of Hamiltonians $H_{s}=H(\cdot, s)$ becomes constant for $|s| \geq R$, and

$$
H_{s}=H_{\alpha^{ \pm}} \in C^{\infty}(V), \quad \pm s \geq R .
$$

Theorem 4.8 For $\epsilon>0$ there exists a constant $\Delta(\epsilon)>0$ with the following property. Assume that $\Sigma^{-}, \Sigma^{+} \subset V$ are stable hypersurfaces such that

$$
\Delta\left(\Sigma^{-}, \Sigma^{+}\right) \leq \Delta(\epsilon) .
$$

Then there exist admissible quintuples $\alpha^{ \pm} \in \mathfrak{A}\left(\Sigma^{ \pm}, V\right)$, a time-dependent Hamiltonian $H \in \mathcal{H}\left(\alpha^{-}, \alpha^{+}\right)$and a time-dependent metric $m=\left\{m_{s}\right\}_{s \in \mathbb{R}}$ on $\mathcal{L} \times \mathbb{R}$ which is constant for $|s|$ large such that the following holds true. For every $a \leq b$ and for every flow line $w=(v, \eta) \in C^{\infty}(\mathbb{R}, \mathcal{L} \times \mathbb{R})$ of the time-dependent gradient $\nabla_{m_{s}} \mathcal{A}^{H_{s}}$ converging asymptotically $\lim _{s \rightarrow \pm \infty} w(s)=w^{ \pm}$to critical points of $\mathcal{A}^{\alpha^{ \pm}}$satisfying

$$
\mathcal{A}^{\alpha^{ \pm}}\left(w^{ \pm}\right), \mathcal{A}_{\alpha^{ \pm}}\left(w^{ \pm}\right) \in[a, b]
$$

the following $L^{\infty}$-estimate holds for $\eta$ :

$$
\|\eta\|_{\infty} \leq\left(\frac{5}{2}+\epsilon\right)^{2}(b-a)+2 .
$$

Proof Assume that $\Sigma^{-}, \Sigma^{+}$are stable hypersurfaces whose stable pseudodistance is $\Delta$. Hence for every $\epsilon_{0}>0$ there exists a path $\mathfrak{P}=\mathfrak{P}_{\epsilon_{0}}=\left\{\mathfrak{S}_{\zeta}\right\}$ of stable quadruples 
$\mathfrak{S}_{\zeta}=\left(\Sigma_{\zeta}, \lambda_{\zeta}, \tau_{\zeta}, I_{\zeta}\right)$ for $\zeta \in[0,1]$ such that

$$
\Delta(\mathfrak{P}) \leq \Delta+\epsilon_{0}
$$

and

$$
\Sigma_{0}=\Sigma^{-}, \quad \Sigma_{1}=\Sigma^{+} .
$$

Let $\rho>1$ be such that $\tau_{\zeta}=\left(\rho, \psi_{\zeta}\right)$. Recall from Section 4.2.2 the spaces $\mathcal{F}_{1}(\rho)$, $\mathcal{F}_{2}(\gamma, \epsilon)$ and $\mathcal{F}_{3}$. Choose

$$
\phi \in \mathcal{F}_{1}(\rho), \quad \chi \in \mathcal{F}_{3} .
$$

Moreover, assume that $\epsilon_{0} \leq 2 / 5$ and pick

$$
h \in \mathcal{F}_{2}\left(\frac{3}{5}, \epsilon_{0}\right) .
$$

Then the family

$$
\alpha_{\zeta}=\left(\lambda_{\zeta}, \tau_{\zeta}, \phi, h, \chi\right), \quad \zeta \in[0,1]
$$

is a smooth family of $\left(3 / 5, \epsilon_{0}\right)$-admissible quintuples for $\Sigma_{\zeta}$. Choose further a smooth function

$$
\zeta_{1} \in C^{\infty}(\mathbb{R},[0,1])
$$

such that

$$
0 \leq \zeta_{1}^{\prime}(s) \leq 1, \quad s \in \mathbb{R}
$$

and

$$
\zeta_{1}(s)= \begin{cases}0 & s \leq-1, \\ 1 & s \geq 1 .\end{cases}
$$

For $R>0$ define $\zeta_{R} \in C^{\infty}(\mathbb{R},[0,1])$ by

$$
\zeta_{R}(s)=\zeta_{1}\left(\frac{s}{R}\right), \quad s \in \mathbb{R} .
$$

Note that

$$
\left\|\zeta_{R}^{\prime}\right\| \leq \frac{1}{R}
$$

Set

$$
\alpha^{-}=\alpha_{0}, \quad \alpha^{+}=\alpha_{1}
$$

and define

$$
H^{R} \in \mathcal{H}\left(\alpha^{-}, \alpha^{+}\right)
$$

by

$$
H_{s}^{R}=H_{\alpha_{\zeta_{R}(s)}}, \quad s \in \mathbb{R} .
$$

To define the time-dependent metric $m=\left\{m_{s}\right\}_{s \in \mathbb{R}}$ choose a smooth family

$$
J_{\zeta} \in \mathcal{J}\left(\alpha_{\zeta}, I_{\zeta}\right) \text {. }
$$

If $(v, \eta) \in \mathcal{L} \times \mathbb{R}$ and $\left(\hat{v}_{1}, \hat{\eta}_{1}\right),\left(\hat{v}_{2}, \hat{\eta}_{2}\right) \in T_{(v, \eta)}(\mathcal{L} \times \mathbb{R})$ we set

$$
m_{s}^{R}\left(\left(\widehat{v}_{1}, \hat{\eta}_{1}\right),\left(\widehat{v}_{2}, \hat{\eta}_{2}\right)\right):=\int_{0}^{1} \omega\left(\widehat{v}_{1}, J_{\zeta_{R}(s)} \widehat{v}_{2}\right) d t+\widehat{\eta}_{1} \widehat{\eta}_{2}
$$


Now assume that $w=(v, \eta) \in C^{\infty}(\mathbb{R}, \mathcal{L} \times \mathbb{R})$ is a gradient flow line of $\nabla_{m_{s}^{R}} \mathcal{A}^{H_{s}^{R}}$. We show in four steps that for $R$ large enough and $\Delta$ small enough the required $L^{\infty}$-bound on $\eta$ holds.

Step 1 For every $\sigma \in \mathbb{R}$ the following inequalities hold:

$$
a-2\left(\Delta+\epsilon_{0}\right)\|\eta\|_{\infty} \leq \mathcal{A}^{H_{\sigma}^{R}}(w(\sigma)) \leq b+2\left(\Delta+\epsilon_{0}\right)\|\eta\|_{\infty} .
$$

We first claim that for any $y \in V$ the estimate

$$
\left|\partial_{s} \bar{H}_{S}^{R}(y)\right| \leq 2 \partial_{s} \zeta_{R}(s) \mathfrak{V}_{\mathfrak{P}}\left(\zeta_{R}(s)\right)
$$

holds. This is clear if $y$ does not lie in the stable tubular neighbourhood $U_{\tau_{\zeta_{R}(s)}}$ since there $\partial_{s} \bar{H}_{S}^{R}$ vanishes. On the other hand, if $y=\psi_{\zeta_{R}(s)}(x, r) \in U_{\tau_{\zeta_{R}}(s)}$, then

$$
\left|\partial_{s} \bar{H}_{s}^{R}(y)\right|=\left|h^{\prime}(r) \partial_{s} \zeta_{R}(s) \omega\left(X_{\psi_{\zeta_{R}}(s)}, R_{\lambda_{\zeta_{R}(s)}}\right)\right| \leq\left(1+\epsilon_{0}\right) \partial_{s} \zeta_{R}(s) \mathfrak{V}_{\mathfrak{P}}\left(\zeta_{R}(s)\right)
$$

which implies (16) since $\epsilon_{0}$ is already chosen to be less than or equal $2 / 5$. We estimate using (16)

$$
\begin{aligned}
& \mathcal{A}^{H_{\sigma}^{R}}(w(\sigma))-\mathcal{A}^{\alpha^{-}}\left(w^{-}\right)=\int_{-\infty}^{\sigma} \frac{d}{d s}\left(\mathcal{A}^{H_{s}^{R}}(w(s))\right) d s \\
& =\int_{-\infty}^{\sigma}\left(\partial_{s} \mathcal{A}^{H_{s}^{R}}\right)(w(s)) d s \\
& +\int_{-\infty}^{\sigma} d \mathcal{A}^{H_{s}^{R}}(w(s)) \partial_{s} w(s) d s \\
& =-\int_{-\infty}^{\sigma} \int_{0}^{1} \eta(s)\left(\partial_{s} H_{s}^{R}\right)(v(s, t)) d t d s \\
& +\int_{-\infty}^{\sigma} m_{s}^{R}\left(\nabla_{m_{s}^{R}} \mathcal{A}^{H_{s}^{R}}\left(w(s), \partial_{s} w(s)\right) d s\right. \\
& \geq-2\|\eta\|_{\infty} \int_{-\infty}^{\sigma} \partial_{S} \zeta_{R}(s) \mathfrak{V}_{\mathfrak{P}}\left(\zeta_{R}(s)\right) d s \\
& +\int_{-\infty}^{\sigma} m_{s}^{R}\left(\partial_{s} w(s), \partial_{s} w(s)\right) d s \\
& \geq-2\|\eta\|_{\infty} \int_{-\infty}^{\infty} \partial_{s} \zeta_{R}(s) \mathfrak{V}_{\mathfrak{P}}\left(\zeta_{R}(s)\right) d s \\
& =-2\|\eta\|_{\infty} \int_{0}^{1} \mathfrak{V}_{\mathfrak{P}}(\zeta) d \zeta \\
& =-2\|\eta\|_{\infty} \Delta(\mathfrak{P}) \\
& =-2\|\eta\|_{\infty}\left(\Delta+\epsilon_{0}\right) \text {. }
\end{aligned}
$$


Now using the fact that $\mathcal{A}^{\alpha^{-}}\left(w^{-}\right) \geq a$ shows the estimate from below in (15). The estimate from above is derived in a similar manner. This finishes the proof of Step 1. To formulate Step 2 we recall that the energy of $w$ with respect to the metric $m_{s}^{R}$ is defined as

$$
E(w)=\int_{-\infty}^{\infty} m_{s}^{R}\left(\partial_{s} w(s), \partial_{s} w(s)\right) d s
$$

Step 2 There exists a constant $c_{\mathfrak{P}}$ such that for every $\sigma \in \mathbb{R}$ the inequalities

$$
\begin{aligned}
& a-4\left(\Delta+\epsilon_{0}\right)\|\eta\|_{\infty}-\frac{c_{\mathfrak{P}}(E(w)+1)}{\sqrt{R}} \leq \mathcal{A}_{\alpha_{\zeta_{R}(\sigma)}}(w(\sigma)), \\
& \mathcal{A}_{\alpha_{\zeta_{R}(\sigma)}}(w(\sigma)) \leq b+4\left(\Delta+\epsilon_{0}\right)\|\eta\|_{\infty}+\frac{c_{\mathfrak{P}}(E(w)+1)}{\sqrt{R}}
\end{aligned}
$$

are satisfied.

For the extension of the stabilizing 1 -form $\lambda_{\zeta^{R}(s)}$ we use the abbreviation

$$
\beta_{s}^{R}=\beta_{\lambda_{\zeta R(s)}}
$$

We claim that for any $y \in V$ the following estimate holds:

$$
\left|\partial_{s} \beta_{s}^{R}\left(X_{\bar{H}_{s}^{R}}(y)\right)\right| \leq 4 \partial_{s} \zeta^{R}(s) \mathfrak{V}_{\mathfrak{P}}\left(\zeta^{R}(s)\right) .
$$

If $y \notin U_{\tau_{\zeta} R_{(s)}}$, then $\beta_{s}^{R}$ vanishes and the estimate obviously holds. We abbreviate

$$
\dot{\beta}_{s}^{R}=\partial_{\zeta^{R}} \beta_{s}^{R} .
$$

Denoting by $L$ the Lie derivative we compute for $y=\psi_{\zeta_{R}(s)}(x, r) \in U_{\tau_{\zeta_{R}}(s)}$

$$
\begin{aligned}
& \dot{\beta}_{s}^{R}\left(X_{\bar{H}_{s}^{R}}(y)\right)=h^{\prime}(r) \dot{\beta}_{s}^{R}\left(R_{\lambda_{\zeta} R(s)}\right) \\
& =h^{\prime}(r) L_{X_{\psi_{\zeta} R_{(s)}}} \beta_{s}^{R}\left(R_{\lambda_{\zeta^{R}(s)}}\right)+h^{\prime}(r) \phi(r) \dot{\lambda}_{\zeta^{R}(s)}\left(R_{\left.\lambda_{\zeta R_{(s)}}\right)}\right) \\
& =h^{\prime}(r) d \iota_{X_{\psi_{\zeta} R_{(s)}}} \beta_{s}^{R}\left(R_{\lambda_{\zeta} R_{(s)}}\right)+h^{\prime}(r) \iota_{X_{\psi_{\zeta} R_{(s)}}} d \beta_{s}^{R}\left(R_{\lambda_{\zeta} R_{(s)}}\right) \\
& +h^{\prime}(r) \phi(r) \dot{\lambda}_{\zeta^{R}(s)}\left(R_{\lambda_{\zeta R(s)}}\right) \\
& =h^{\prime}(r) d\left(\phi(r) \iota_{X_{\psi_{\zeta} R_{(s)}}} \lambda_{\zeta^{R}(s)}\right)\left(R_{\lambda_{\zeta} R_{(s)}}\right) \\
& +h^{\prime}(r) \iota_{X_{\psi_{\zeta} R_{(s)}}}\left(\phi^{\prime}(r) d r \wedge \lambda_{\zeta^{R}(s)}+\phi(r) d \lambda_{\zeta^{R}(s)}\right)\left(R_{\left.\lambda_{\zeta R(s)}\right)}\right) \\
& +h^{\prime}(r) \phi(r) \dot{\lambda}_{\zeta^{R}(s)}\left(R_{\left.\lambda_{\zeta R_{(s)}}\right)}\right.
\end{aligned}
$$




$$
\begin{aligned}
& =h^{\prime}(r) \phi(r) d\left(\iota_{X_{\psi_{\zeta} R_{(s)}}} \lambda_{\zeta R^{R}(s)}\right)\left(R_{\lambda_{\zeta} R_{(s)}}\right) \\
& +h^{\prime}(r) \phi^{\prime}(r) \iota_{X_{\psi_{\zeta} R_{(s)}}} \lambda_{\zeta R_{(s)}} d r\left(R_{\lambda_{\zeta} R_{(s)}}\right) \\
& +h^{\prime}(r) \iota_{X_{\psi_{\zeta} R_{(s)}}}\left(\omega_{\Sigma}+\phi^{\prime}(r) d r \wedge \lambda_{\zeta^{R}(s)}+\phi(r) d \lambda_{\zeta{ }^{R}(s)}\right)\left(R_{\lambda_{\zeta R_{(s)}}}\right) \\
& +h^{\prime}(r) \phi(r) \dot{\lambda}_{\zeta R(s)}\left(R_{\lambda_{\zeta} R_{(s)}}\right) \\
& =h^{\prime}(r) \phi(r) d\left(\iota_{X_{\psi_{\zeta} R_{(s)}}} \lambda_{\zeta{ }^{R}(s)}\right)\left(R_{\lambda_{\zeta R_{(s)}}}\right) \\
& +h^{\prime}(r) \omega\left(X_{\psi_{\zeta} R_{(s)}}, R_{\lambda_{\zeta} R_{(s)}}\right) \\
& +h^{\prime}(r) \phi(r) \dot{\lambda}_{\zeta R_{(s)}}\left(R_{\lambda_{\zeta} R_{(s)}}\right) \text {. }
\end{aligned}
$$

In the fifth equality we have used that the Reeb vector field lies in the kernel of $\omega_{\Sigma}$. Using

$$
\max \phi \leq \rho+1 \leq 2 \rho, \quad \max h^{\prime} \leq 1+\epsilon_{0} \leq 2
$$

we obtain from that the estimate

$$
\left|\dot{\beta}_{S}^{R}\left(X_{\bar{H}_{S}^{R}}(y)\right)\right| \leq 4 \mathfrak{V}_{\mathfrak{P}}\left(\zeta_{R}(s)\right)
$$

implying (18).

As in the time-independent case we consider the following time-dependent bilinear form on $T(\mathcal{L} \times \mathbb{R})$

$$
\hat{m}_{s}^{R}\left(\left(\widehat{v}_{1}, \hat{\eta}_{1}\right),\left(\hat{v}_{2}, \hat{\eta}_{2}\right)\right):=\int_{0}^{1} d \beta_{s}^{R}\left(\widehat{v}_{1}, J_{\zeta_{R}(s)} \widehat{v}_{2}\right) d t+\widehat{\eta}_{1} \hat{\eta}_{2}
$$

By Proposition 4.1 we have for any $s \in \mathbb{R}$

$$
\nabla_{m_{s}^{R}} \mathcal{A}^{H_{s}^{R}}=\nabla_{\hat{m}_{s}^{R}} \hat{\mathcal{A}}^{H_{s}^{R}} .
$$

Hence the computation in Corollary 4.2 shows that

$$
\text { (19) } \begin{aligned}
\frac{d}{d s}\left(\mathcal{A}_{\alpha_{\zeta_{R}(s)}}(w(s))\right)= & \left(\partial_{s} \mathcal{A}_{\alpha_{\zeta_{R}(s)}}\right)(w(s)) \\
& +\left(m_{s}^{R}-\widehat{m}_{s}^{R}\right)\left(\nabla_{m_{s}^{R}} \mathcal{A}^{H_{s}^{R}}(w(s)), \nabla_{m_{s}^{R}} \mathcal{A}^{H_{s}^{R}}(w(s))\right) \\
\geq & \left(\partial_{s} \mathcal{A}_{\alpha_{\zeta_{R}(s)}}\right)(w(s)) .
\end{aligned}
$$

For $\zeta \in[0,1]$ let $\gamma_{\zeta}$ be the compactly supported 1 -form

$$
\gamma_{\zeta}=\dot{\beta}_{\lambda_{\zeta}} \circ J_{\zeta}
$$


and define the constant $c_{\mathfrak{P}}$ as

$$
c_{\mathfrak{P}}=\max _{\substack{\zeta \in[0,1] \\ y \in V}}\left\|\gamma_{\zeta}(y)\right\|
$$

where the norm is taken with respect to the metric $\omega\left(\cdot, J_{\zeta} \cdot\right)$. Again we only show the estimate from below. This is derived similarly as the estimate (17) in Step 1. Using (18) and (19) we obtain

$$
\begin{aligned}
& \mathcal{A}_{\alpha_{\zeta_{R}(\sigma)}}(w(\sigma))-\mathcal{A}_{\alpha^{-}}\left(w^{-}\right) \geq-\int_{-\infty}^{\sigma}\left(\partial_{s} \mathcal{A}_{\alpha_{\zeta_{R}(s)}}\right)(w(s)) d s \\
& =-\int_{-\infty}^{\sigma} \partial_{s} \zeta_{R}(s)\left(\int_{0}^{1} v^{*} \dot{\beta}_{s}^{R} d t\right) d s \\
& =-\int_{-\infty}^{\sigma} \partial_{s} \zeta_{R}(s) \eta(s)\left(\int_{0}^{1} \dot{\beta}_{s}^{R}\left(\eta X_{H_{s}^{R}}(v(s, t))\right) d t\right) d s \\
& -\int_{-\infty}^{\sigma} \partial_{s} \zeta_{R}(s)\left(\int_{0}^{1} \dot{\beta}_{s}^{R}\left(J_{\zeta_{R}(s)} \partial_{s} v(s, t)\right) d t\right) d s \\
& \geq-4\|\eta\|_{\infty} \int_{-\infty}^{\sigma} \partial_{s} \zeta_{R}(s) \mathfrak{V}_{\mathfrak{P}}\left(\zeta_{R}(s)\right) d s \\
& -c_{\mathfrak{P}} \int_{-\infty}^{\sigma} \int_{0}^{1} \partial_{s} \zeta_{R}(s)\left\|\partial_{s} v(s, t)\right\| d t d s \\
& \geq-4\|\eta\|_{\infty} \int_{-\infty}^{\infty} \partial_{s} \zeta_{R}(s) \mathfrak{V}_{\mathfrak{P}}\left(\zeta_{R}(s)\right) d s \\
& -c_{\mathfrak{P}} \int_{-\infty}^{\infty} \partial_{s} \zeta_{R}(s) \int_{0}^{1}\left(\sqrt{R}\left\|\partial_{s} v(s, t)\right\|^{2}+\frac{1}{\sqrt{R}}\right) d t d s \\
& \geq-4\|\eta\|_{\infty} \int_{0}^{1} \mathfrak{V}_{\mathfrak{P}}(\zeta) d \zeta-\frac{c_{\mathfrak{P}}}{\sqrt{R}} \int_{-\infty}^{\infty} \partial_{s} \zeta_{R}(s) d s \\
& -\frac{c_{\mathfrak{P}}}{\sqrt{R}} \int_{-\infty}^{\infty}\left\|\partial_{s} w(s)\right\|^{2} d s \\
& \geq-4\|\eta\|_{\infty}\left(\Delta+\epsilon_{0}\right)-\frac{c_{\mathfrak{P}}(E(w)+1)}{\sqrt{R}} .
\end{aligned}
$$

This finishes the proof of Step 2.

Step 3 The energy can be estimated by

$$
E(w) \leq \mathcal{A}^{\alpha^{+}}\left(w^{+}\right)-\mathcal{A}^{\alpha^{-}}\left(w^{-}\right)+2\|\eta\|_{\infty}\left(\Delta+\epsilon_{0}\right) \leq b-a+2\|\eta\|_{\infty}\left(\Delta+\epsilon_{0}\right) .
$$

A careful look at (17) reveals Step 3. 
Step 4 We prove the theorem.

As in the proof of Proposition 4.4, we set for $\sigma \in \mathbb{R}$

$$
\tau(\sigma)=\inf \left\{\tau \geq 0:\left\|\nabla \mathcal{A}^{H_{\sigma}^{R}}(w(\sigma+\tau))\right\| \leq \frac{2}{5}\right\} .
$$

In the time dependent case we still can estimate

$$
\tau(\sigma) \leq \frac{25 E(w)}{4}
$$

Since $\alpha_{\zeta}$ is $\left(3 / 5, \epsilon_{0}\right)$-admissible for every $\zeta \in[0,1]$ we conclude from Lemma 4.7 applied to $\gamma=3 / 5$ together with the fact that $\left\|H_{s}^{R}\right\|_{\infty} \leq 3 / 5+\epsilon_{0}$ for every $s \in \mathbb{R}$ and (20) the following estimate

$$
\begin{aligned}
|\eta(\sigma)| & \leq|\eta(\sigma+\tau(\sigma))|+\int_{\sigma}^{\sigma+\tau(\sigma)}\left|\partial_{s} \eta(s)\right| d s \\
& \leq \frac{5}{2} \sup \left|\hat{\mathcal{A}}^{H^{R}} \circ w\right|+1+\tau(\sigma)\left\|H^{R}\right\|_{\infty} \\
& \leq \frac{5}{2} \sup \left|\hat{\mathcal{A}}^{H^{R}} \circ w\right|+1+\frac{25 E(w)}{4}\left(\frac{3}{5}+\epsilon_{0}\right) .
\end{aligned}
$$

From Steps 1 and 2 we obtain the estimate

$$
\begin{aligned}
\sup \left|\hat{\mathcal{A}}^{H^{R}} \circ w\right| & =\sup \left|\mathcal{A}^{H^{R}} \circ w-\mathcal{A}_{\alpha_{\zeta_{R}}} \circ w\right| \\
& \leq b-a+6\left(\Delta+\epsilon_{0}\right)\|\eta\|_{\infty}+\frac{c_{\mathfrak{P}}(E(w)+1)}{\sqrt{R}} .
\end{aligned}
$$

Combining (21) and (22) with Step 3 we obtain

$$
\begin{aligned}
\|\eta\|_{\infty} \leq & \frac{5}{2}(b-a)+1+\frac{5 c_{\mathfrak{P}}}{2 \sqrt{R}}+\left(\frac{15}{4}+\frac{25 \epsilon_{0}}{4}+\frac{5 c_{\mathfrak{P}}}{2 \sqrt{R}}\right) E(w) \\
& +15\left(\Delta+\epsilon_{0}\right)\|\eta\|_{\infty} \\
\leq & \left(\frac{25\left(1+\epsilon_{0}\right)}{4}+\frac{c_{\mathfrak{P}}}{\sqrt{R}}\right)(b-a)+1+\frac{5 c_{\mathfrak{P}}}{2 \sqrt{R}} \\
& +\left(\frac{45}{2}+\frac{25 \epsilon_{0}}{2}+\frac{5 c_{\mathfrak{P}}}{\sqrt{R}}\right)\left(\Delta+\epsilon_{0}\right)\|\eta\|_{\infty} .
\end{aligned}
$$

We now choose $\Delta=\Delta(\epsilon)$ in such a way that

$$
0<\Delta<\min \left\{\frac{2 \epsilon}{125+100 \epsilon}, \frac{1}{60}\right\} .
$$


The positive number $\epsilon_{0}$ which already had to be chosen in such a way that $\epsilon_{0} \leq 2 / 5$ is now supposed to satisfy

$$
0<\epsilon_{0} \leq \min \left\{\frac{1}{60}-\Delta, \frac{2 \epsilon}{125+100 \epsilon}-\Delta\right\} .
$$

Moreover, we choose $R$ such that

$$
R \geq \max \left\{\left(\frac{5 c_{\mathfrak{P}}}{2\left(1-60\left(\Delta+\epsilon_{0}\right)\right)}\right)^{2},\left(\frac{c_{\mathfrak{P}}}{\epsilon}\right)^{2}\right\} .
$$

With these choices we get from (23)

$$
\|\eta\|_{\infty} \leq\left(\frac{25}{4}+2 \epsilon\right)(b-a)+2\left(1-30\left(\Delta+\epsilon_{0}\right)\right)+30\left(\Delta+\epsilon_{0}\right)\|\eta\|_{\infty} .
$$

Using $\Delta+\epsilon_{0} \leq 2 \epsilon /(125+100 \epsilon)$ we obtain

$$
\begin{aligned}
\|\eta\|_{\infty} & \leq \frac{1}{1-30\left(\Delta+\epsilon_{0}\right)}\left(\frac{25}{4}+2 \epsilon\right)(b-a)+2 \\
& \leq\left(\frac{25}{4}+5 \epsilon\right)(b-a)+2 \\
& \leq\left(\frac{5}{2}+\epsilon\right)^{2}(b-a)+2 .
\end{aligned}
$$

This finishes the proof of the theorem.

\subsection{An existence result for a periodic Reeb orbit}

In this subsection we show how compactness for gradient flow lines of Rabinowitz action functional leads to existence of a periodic Reeb orbit on stable displaceable hypersurfaces. This result is not new. Indeed, Schlenk proved it before in [64] using quite different methods. Before stating the theorem we recall some well-known notions.

A hypersurface $\Sigma$ in a symplectic manifold $(V, \omega)$ is called displaceable, if there exists a compactly supported Hamiltonian $F \in C^{\infty}\left(V \times S^{1}\right)$ such that the time-one flow $\phi_{F}$ of the time dependent Hamiltonian vector field $X_{F_{t}}$ with $F_{t}=F(\cdot, t) \in C^{\infty}(V)$ satisfies

$$
\phi_{F}(\Sigma) \cap \Sigma=\varnothing .
$$

The positive and the negative part of the Hofer norm for the compactly supported Hamiltonian $F$ are given by

$$
\|F\|_{+}=\int_{0}^{1} \max _{V} F_{t} d t, \quad\|F\|_{-}=-\int_{0}^{1} \min _{V} F_{t} d t
$$


and the Hofer norm itself by

$$
\|F\|=\|F\|_{+}+\|F\|_{-} .
$$

If $\Sigma \subset V$ is a displaceable hypersurface its displacement energy is given by

$$
e(\Sigma)=\inf \left\{\|F\|: \phi_{F}(\Sigma) \cap \Sigma=\varnothing\right\} .
$$

Recall from (2) the $\omega$-energy for closed characteristics.

Theorem 4.9 (Schlenk) Assume that $\Sigma$ is a stable, displaceable hypersurface in a symplectically aspherical, geometrically bounded, symplectic manifold $(V, \omega)$. Then $\Sigma$ has a closed characteristic $v$ which is contractible in $V$ and satisfies

$$
\Omega(v) \leq e(\Sigma) .
$$

Our proof of Theorem 4.9 is based on a homotopy stretching argument for a time dependent perturbation of Rabinowitz action functional where the perturbation is given by the displacing Hamiltonian.

The crucial point is that the perturbed Rabinowitz action functional has no critical points anymore. This is actually true for any separating closed hypersurface $\Sigma$ in a symplectically aspherical, symplectic manifold $(V, \omega)$. If $\Sigma=\bar{H}^{-1}(0)$ for a Hamiltonian $\bar{H} \in C^{\infty}(V)$ we choose $\chi \in C^{\infty}\left(S^{1}, \mathbb{R}\right)$ of integral one which in addition meets the condition

$$
\operatorname{supp} \chi \subset(0,1 / 2)
$$

and set as usual

$$
H(t, y)=\chi(t) \bar{H}(y), \quad y \in V, t \in S^{1} .
$$

Without changing the Hofer norm we furthermore can reparametrize the flow of the displacing Hamiltonian $F_{t}$ such that we can assume that

$$
F_{t}=0, \quad t \in[0,1 / 2] \text {. }
$$

The perturbation of Rabinowitz action functional which we consider is the functional $\mathcal{A}_{F}^{H}: \mathcal{L} \times \mathbb{R} \rightarrow \mathbb{R}$ defined by

$$
\mathcal{A}_{F}^{H}(v, \eta)=\mathcal{A}^{H}(v, \eta)-\int_{0}^{1} F_{t}(v(t)) d t, \quad(v, \eta) \in \mathcal{L} \times \mathbb{R} .
$$

We further denote by

$$
\mathfrak{S}\left(X_{\bar{H}}\right)=\operatorname{cl}\left\{y \in V: X_{\bar{H}}(y) \neq 0\right\}
$$


the support of the Hamiltonian vector field of $\bar{H}$. The following Lemma is proved in [13] where it appears as Lemma 3.10. It immediately implies that a suitable perturbed Rabinowitz action functional has no critical points anymore.

Lemma 4.10 Assume that $\mathfrak{S}\left(X_{\bar{H}}\right)$ is compact and $\phi_{F}\left(\mathfrak{S}\left(X_{\bar{H}}\right)\right) \cap \mathfrak{S}\left(X_{\bar{H}}\right)=\varnothing$. Then for every $\omega$-compatible almost complex structure $J$ on $V$ there exists a constant $\mu=\mu(J)>0$ such that if $\nabla$ is the gradient and $\|\cdot\|$ is the norm of the metric on $\mathcal{L} \times \mathbb{R}$ induced from $J$, then for every $(v, \eta) \in \mathcal{L} \times \mathbb{R}$,

$$
\left\|\nabla \mathcal{A}_{F}^{H}(v, \eta)\right\| \geq \mu .
$$

With the help of this Lemma we are now armed for the proof of Schlenk's Theorem.

Proof of Theorem 4.9 Given $\epsilon_{0}>0$ we choose a time dependent Hamiltonian $F_{t}$ satisfying the conditions

$$
F_{t}=0, t \in[0,1 / 2], \quad \phi_{F}(\Sigma) \cap \Sigma=\varnothing, \quad\|F\|<e(\Sigma)+\epsilon_{0} .
$$

We further choose a $(\gamma, \epsilon)$-admissible quintuple $\alpha=(\lambda, \tau, \phi, h, \chi)$ which satisfies

$$
\operatorname{supp} \chi \subset(0,1 / 2), \quad \phi_{F}\left(U_{\tau}\right) \cap U_{\tau}=\varnothing .
$$

The first condition can obviously be achieved. To see that one can also assume without loss of generality the second condition we note that since $\Sigma$ is compact there exists an open neighbourhood $U_{\Sigma}$ of $\Sigma$ in $V$ which is also displaced by $\phi_{F}$. Now by choosing $\lambda$ arbitrarily small we can arrange that even a large tubular neighbourhood $U_{\tau}$ is contained in $U_{\Sigma}$.

We further choose a smooth family of cutoff functions $\beta_{r} \in C^{\infty}(\mathbb{R},[0,1])$ for $r \in[0, \infty)$ with the properties

$$
\left\{\begin{aligned}
\beta_{r}(s)=0 & |s| \geq r, \\
\beta_{r}(s)=1 & |s| \leq r-1, \\
s \beta_{r}^{\prime}(s) \leq 0 & \forall r, \forall s .
\end{aligned}\right.
$$

We now consider the $r$-parametrised family of time dependent perturbed Rabinowitz action functionals defined by

$$
\mathcal{A}_{r}^{\alpha}(v, \eta, s)=\mathcal{A}^{\alpha}(v, \eta)-\beta_{r}(s) \int_{0}^{1} F_{t}(v(t)) d t, \quad v \in \mathcal{L}, \eta \in \mathbb{R}, s \in \mathbb{R}, r \in[0, \infty) .
$$

We note that

$$
\mathcal{A}_{0}^{\alpha}=\mathcal{A}^{\alpha}
$$

is independent of the $s$-variable. We further choose an $\omega$-compatible almost complex structure $J \in \mathcal{J}(\alpha)$ and denote by $\nabla$ the gradient with respect to the metric $m$ on 
$\mathcal{L} \times \mathbb{R}$ induced from $J$. We fix a point $x \in \Sigma$ and think of it as a loop in $\mathcal{L}$. We are studying solutions $(w, r)=(v, \eta, r) \in C^{\infty}(\mathbb{R}, \mathcal{L} \times \mathbb{R}) \times[0, \infty)$ of the problem

(25) $\partial_{s} w(s)=\nabla \mathcal{A}_{r}^{\alpha}(s, w(s)), s \in \mathbb{R}, \quad \lim _{s \rightarrow-\infty} w(s)=(x, 0), \quad \lim _{s \rightarrow \infty} w(s) \in \Sigma \times\{0\}$.

We use the following abbreviation for its moduli space

$$
\mathcal{M}=\{(w, r):(w, r) \text { solution of }(25)\} .
$$

To prove the Theorem we argue by contradiction and assume

$$
\Omega(v)>\|F\|, \quad \forall v \in X(\Sigma) .
$$

To see how this leads to a contradiction, we first show the following claim.

Claim If (26) holds, then $\mathcal{M}$ is compact.

We prove the Claim in four steps. For the first step recall that the energy of $w$ is defined by

$$
E(w)=\int_{-\infty}^{\infty}\left\|\partial_{s} w\right\|^{2} d s
$$

where the norm is taken with respect to the metric $m$ induced from the $\omega$-compatible almost complex structure $J$.

Step 1 If $(w, r) \in \mathcal{M}$ then $E(w) \leq\|F\|$.

We estimate using (25)

$$
\begin{aligned}
E(w) & =\int_{-\infty}^{\infty} d \mathcal{A}_{r}^{\alpha}(w)\left(\partial_{s} w\right) d s \\
& =\int_{-\infty}^{\infty} \frac{d}{d s} \mathcal{A}_{r}^{\alpha}(w) d s-\int_{-\infty}^{\infty}\left(\partial_{s} \mathcal{A}_{r}^{\alpha}\right)(w) d s \\
& =0+\int_{-\infty}^{\infty} \beta_{r}^{\prime}(s)\left(\int_{0}^{1} F_{t}(v) d t\right) d s \\
& \leq\|F\|_{+} \int_{-\infty}^{0} \beta_{r}^{\prime}(s) d s-\|F\|_{-} \int_{0}^{\infty} \beta_{r}^{\prime}(s) d s \\
& \leq\|F\|_{+}+\|F\|_{-} \\
& =\|F\| .
\end{aligned}
$$

This finishes the proof of Step 1.

Step 2 There exists $r_{0} \in \mathbb{R}$ such that if $(w, r) \in \mathcal{M}$ then $r \leq r_{0}$. 
Combining Lemma 4.10 with Step 1 we obtain the estimate

$$
\begin{aligned}
\|F\| & \geq \int_{-r}^{r}\left\|\nabla \mathcal{A}_{F}^{\alpha}(w)\right\|^{2} d s \\
& \geq 2 \mu^{2} r
\end{aligned}
$$

implying that

$$
r \leq \frac{\|F\|}{2 \mu^{2}}=: r_{0} .
$$

This finishes the proof of Step 2.

Step 3 There exists a constant $c>0$ such that for all $(w, r)=(v, \eta, r) \in \mathcal{M}$ the Lagrange multiplier $\eta$ is uniformly bounded by $\|\eta\| \leq c$.

To prove Step 3 we estimate the functional $\mathcal{A}=\mathcal{A}^{\alpha}-\widehat{\mathcal{A}}^{\alpha}$ along $w$. Note that we do not perturb $\mathcal{A}$ with the displacing Hamiltonian $F$. It is useful to introduce further the functional

$$
\mathcal{F}: \mathcal{L} \times \mathbb{R} \rightarrow \mathbb{R}, \quad(v, \eta) \mapsto \int_{0}^{1} F_{t}(v(t)) d t
$$

which actually only depends on the first variable. By Proposition 4.1 we have

$$
\nabla_{\widehat{m}} \hat{\mathcal{A}}^{\alpha}=\nabla_{m} \mathcal{A}^{\alpha} \text {. }
$$

Hence using (25) we estimate similarly as in Corollary 4.2

$$
\begin{aligned}
\frac{d}{d s} \mathcal{A}(w) & =d \mathcal{A}^{\alpha}(w) \partial_{s} w-d \hat{\mathcal{A}}^{\alpha}(w) \partial_{s} w \\
& =m\left(\nabla_{m} \mathcal{A}^{\alpha}(w), \partial_{s} w\right)-\hat{m}\left(\nabla_{m} \mathcal{A}^{\alpha}(w), \partial_{s} w\right) \\
& =(m-\hat{m})\left(\partial_{s} w, \partial_{s} w\right)+\beta_{r}(m-\hat{m})\left(\nabla_{m} \mathcal{F}(w), \partial_{s} w\right) \\
& \geq \beta_{r}(m-\hat{m})\left(\nabla_{m} \mathcal{F}(w), \partial_{s} w\right) .
\end{aligned}
$$

Since $F$ has compact support there exists a constant $c_{0}$ such that for all $w \in \mathcal{L} \times \mathbb{R}$

$$
\left\|(m-\hat{m})\left(\nabla_{m} \mathcal{F}(w), \cdot\right)\right\|_{m} \leq c_{0} .
$$

Hence we obtain for $\sigma \in \mathbb{R}$ using Steps 1 and 2

$$
\begin{aligned}
\mathcal{A}(w(\sigma))=\int_{-\infty}^{\sigma} \frac{d}{d s} \mathcal{A}(w) d s & \geq \int_{-\infty}^{\sigma} \beta_{r}(m-\hat{m})\left(\nabla_{m} \mathcal{F}(w), \partial_{s} w\right) d s \\
& \geq-c_{0} \int_{-r}^{r}\left\|\partial_{s} w\right\|_{m} d s \\
& \geq-c_{0} \int_{-r}^{r}\left(\left\|\partial_{s} w\right\|_{m}^{2}+1\right) d s \\
& \geq-c_{0}(2 r+E(w)) \geq-c_{0}\left(2 r_{0}+\|F\|\right) .
\end{aligned}
$$


Similarly, one gets

$$
-\mathcal{A}(w(\sigma))=\int_{\sigma}^{\infty} \frac{d}{d s} \mathcal{A}(w) d s \leq c_{0}\left(2 r_{0}+\|F\|\right) .
$$

Defining the constant

$$
c_{1}=c_{0}\left(2 r_{0}+\|F\|\right)
$$

we obtain from the previous two estimates the uniform $L^{\infty}$-bound

$$
\|\mathcal{A} \circ w\| \leq c_{1} .
$$

Moreover, a closer look at the estimate in Step 1 reveals that

$$
\left\|\mathcal{A}_{r}^{\alpha} \circ w\right\| \leq\|F\| .
$$

Noting that for $s \notin(-r, r)$ we have $\mathcal{A}_{r}^{\alpha}(\cdot, s)=\mathcal{A}^{\alpha}$ we obtain from the previous two inequalities

$$
\left|\hat{\mathcal{A}}^{\alpha}(w(s))\right| \leq c_{1}+\|F\|, \quad s \in \mathbb{R} \backslash(-r, r) .
$$

The proof for the bound of the Lagrange multiplier now proceeds similarly as in Proposition 4.4. For $\sigma \in \mathbb{R}$ we set

$$
\tau(\sigma)=\inf \left\{\tau: \sigma+\tau \notin(-r, r), \| \nabla \mathcal{A}^{\alpha}\left(w(\sigma+\tau) \| \leq \frac{2 \gamma}{3}\right\} .\right.
$$

Again $\tau(\sigma)$ can be estimated in terms of the energy by

$$
\tau(\sigma) \leq \frac{9 E(w)}{4 \gamma^{2}}+2 r \leq \frac{9\|F\|}{4 \gamma^{2}}+2 r_{0} .
$$

Combining Lemma 4.3 with (27) and (28) and using $\partial_{s} \eta=-\int_{0}^{1} H(v) d t$ we estimate

$$
\begin{aligned}
|\eta(\sigma)| & \leq|\eta(\sigma+\tau(\sigma))|+\int_{\sigma}^{\sigma+\tau(\sigma)}\left|\partial_{s} \eta\right| d s \\
& \leq \frac{3 c_{1}+3\|F\|+2 \gamma}{3(1-\gamma)}+(\gamma+\epsilon) \tau(\sigma) \\
& \leq \frac{3 c_{1}+3\|F\|+2 \gamma}{3(1-\gamma)}+\frac{9\|F\|(\gamma+\epsilon)}{4 \gamma^{2}}+2 r_{0}(\gamma+\epsilon) .
\end{aligned}
$$

Since $\sigma$ was arbitrary we are done with Step 3 .

Step 4 We prove the claim.

For $v \in \mathbb{N}$ let $\left(w_{\nu}, r_{v}\right)=\left(v_{v}, \eta_{\nu}, r_{v}\right)$ be a sequence in $\mathcal{M}$. Since the homotopy parameter $r_{v}$ is uniformly bounded by Step 2 and the Lagrange multiplier $\eta_{v}$ is 
uniformly bounded by Step 3 standard arguments in Floer theory imply that $\left(w_{v}, r_{v}\right)$ has a $C_{\text {loc }}^{\infty}$-convergent subsequence. Indeed, $v_{v}$ satisfies a uniform $C^{0}$-bound by the assumption that $(V, \omega)$ is geometrically bounded and the derivatives of $v_{v}$ can be controlled because there is no bubbling since $(V, \omega)$ is symplectically aspherical. Let $(w, r)$ be the limit of the subsequence. $(w, r)$ obviously satisfies the first equation in (25). It remains to check that $w$ satisfies the asymptotic conditions. Again by compactness it follows that $w(s)$ converges to critical points $w^{ \pm}=\left(v^{ \pm}, \eta^{ \pm}\right)$of $\mathcal{A}^{\alpha}$ as $s$ goes to $\pm \infty$. On the other hand it follows from Step 1 that

$$
\mathcal{A}_{r}^{\alpha}(w(s)) \in[-\|F\|,\|F\|], \quad \forall s \in \mathbb{R}
$$

and hence

$$
\Omega\left(v^{ \pm}\right)=\mathcal{A}^{\alpha}\left(w^{ \pm}\right) \in[-\|F\|,\|F\|] .
$$

Therefore (26) implies that $v^{ \pm}$has to be constant and hence

$$
w^{-}=(x, 0), \quad w^{+} \in \Sigma \times\{0\} .
$$

This finishes the prove of the claim.

Given the claim we are now in position to prove the theorem in a last step.

Step 5 We prove the theorem.

Given the claim it remains to argue that the compactness of the moduli space $\mathcal{M}$ is absurd in order to show that (26) cannot be true. For $r=0$ there is precisely one point $(w, 0) \in \mathcal{M}$ namely the constant gradient flow line $w=(x, 0)$. The constant gradient flow line is regular in the sense that the linearization of the gradient flow equation at it is surjective. Thinking of the moduli space $\mathcal{M}$ as the zero set of a Fredholm section from a Banach space into a Banach bundle and using that it is compact we can perturb this section slightly to make it transverse. The zero set of the perturbed section is now a compact manifold with one single boundary point $(x, 0,0)$. However, such manifolds do not exist. Therefore (26) had to be wrong and we conclude that there exists $v \in X(\Sigma)$ such that

$$
\Omega(v) \leq\|F\|<e(\Sigma)+\epsilon_{0} .
$$

Since $\epsilon_{0}>0$ was arbitrary the theorem follows.

\subsection{Approvable perturbations}

Except in the case where $V$ is zero dimensional, the Rabinowitz action functional is never Morse, since its critical set contains the constant solutions and each nontrivial Reeb orbit comes in an $S^{1}$-family coming from time-shift. The best situation we 
can hope for, is that Rabinowitz action functional is Morse-Bott. However, since the stability condition is not an open condition [15], a slight perturbation of a stable hypersurface might not be stable anymore.

In this subsection we study a class of perturbations of Rabinowitz action functional. We first show that for a generic perturbation the perturbed Rabinowitz action functional is Morse. We then explain how in the weakly tame case for small perturbations the moduli space of gradient flow lines in a fixed action interval can be written as the disjoint union of two closed subspaces where one of them is compact. We refer to the compact part as the essential part of the moduli space of gradient flow lines. We finally explain how the essential part of the moduli space of gradient flow lines can be used to define a boundary operator for a fixed action interval.

The perturbations of Rabinowitz action functional we consider are reminiscent of the ones we considered in the previous subsection, however they are more general, since we do not require that the time support of the additional perturbation Hamiltonian is disjoint from the time support of the Hamiltonian $H_{\alpha}$. Namely we choose a compactly supported time-dependent Hamiltonian $F \in C_{c}^{\infty}\left(V \times S^{1}\right)$ and define $\mathcal{A}_{F}^{\alpha}: \mathcal{L} \times \mathbb{R} \rightarrow \mathbb{R}$ as in the previous subsection by

$$
\mathcal{A}_{F}^{\alpha}(v, \eta)=\mathcal{A}^{\alpha}(v, \eta)-\int_{0}^{1} F_{t}(v(t)) d t .
$$

Critical points of the action functional $\mathcal{A}_{F}^{\alpha}$ are solutions of the problem

$$
\left\{\begin{array}{l}
\partial_{t} v=\eta X_{H}(v)+X_{F_{t}}(v), \\
\int_{0}^{1} H(v(t)) d t=0 .
\end{array}\right.
$$

We first show that for generic perturbations the perturbed action functional is Morse.

Proposition 4.11 Given an admissible quadruple $\alpha$, there exists a subset $\mathcal{U}(\alpha) \in$ $C_{c}^{\infty}\left(V \times S^{1}\right)$ of the second category such that $\mathcal{A}_{F}^{\alpha}$ is Morse for every $F \in \mathcal{U}(\alpha)$.

Proof Consider the Hilbert manifold

$$
\mathcal{H}=W^{1,2}\left(S^{1}, V\right) \times \mathbb{R}
$$

where we define $W^{1,2}\left(S^{1}, V\right)$ by embedding $V$ into $\mathbb{R}^{N}$ for $N$ large enough. Over the Hilbert manifold $\mathcal{H}$ we introduce the Hilbert bundle

$$
\pi: \mathcal{E} \rightarrow \mathcal{H}
$$

whose fibre over $(v, \eta) \in \mathcal{H}$ is given by

$$
\mathcal{E}_{(v, \eta)}=L^{2}\left(S^{1}, v^{*} T V\right) \times \mathbb{R} .
$$


Choose an $\omega$-compatible almost complex structure $J$ and denote by $\nabla$ the gradient with respect to the metric $\omega(\cdot, J \cdot)$. For $F \in C_{c}^{\infty}\left(V \times S^{1}\right)$ we define a section

by

$$
s_{F}(v, \eta)=\left(\begin{array}{c}
s_{F}: \mathcal{H} \rightarrow \mathcal{E} \\
\int_{t} v-\eta \nabla H(v)-\nabla F_{t}(v) \\
\int_{0}^{1} H(v(t)) d t
\end{array}\right) .
$$

Note that the zero set $s_{F}^{-1}(0)$ coincides with the solutions of (29). If $w \in \mathcal{H} \subset \mathcal{E}$ then there is a canonical splitting

We denote by

$$
T_{w} \mathcal{E}=\mathcal{E}_{w} \times T_{w} \mathcal{H}
$$

$$
\Pi_{w}: T_{w} \mathcal{E} \rightarrow \mathcal{E}_{w}
$$

the projection along $T_{w} \mathcal{H}$. If $(v, \eta) \in s_{F}^{-1}(0)$ we introduce the vertical differential

by

$$
\begin{gathered}
D s_{F}(v, \eta): T_{(v, \eta)} \mathcal{H}=W^{1,2}\left(S^{1}, v^{*} T V\right) \times \mathbb{R} \rightarrow \mathcal{E}_{(v, \eta)} \\
D s_{F}(v, \eta)=\Pi_{(v, \eta)} \circ d s_{F}(v, \eta) .
\end{gathered}
$$

The action functional $\mathcal{A}_{F}^{\alpha}$ is Morse if and only if the vertical differential $D s_{F}(w)$ is surjective for every $w \in s_{F}^{-1}(0)$ meaning that $s_{F}$ is transverse to the zero section

$$
s_{F} \pitchfork 0 .
$$

We prove in two steps that (30) holds for generic $F \in C_{c}^{\infty}\left(V \times S^{1}\right)$. In the first step we prove transversality for weaker differentiability assumptions. The smooth case follows then by an argument due to Taubes.

Step 1 Assume that $2 \leq k<\infty$. Then there exists $\mathcal{U}^{k}(\alpha) \subset C_{c}^{k}\left(V \times S^{1}\right)$ of the second category, such that (30) holds for any $F \in \mathcal{U}^{k}(\alpha)$.

Consider the section

$$
S: C_{c}^{k}\left(V \times S^{1}\right) \times \mathcal{H} \rightarrow \mathcal{E}
$$

which is defined by

$$
S(F, w)=s_{F}(w), \quad F \in C_{c}^{k}\left(V \times S^{1}\right), w \in \mathcal{H} .
$$

If $(F, w) \in S^{-1}(0)$ with $w=(v, \eta)$, then the vertical differential

$$
D S(F, w): T_{(F, w)}\left(C_{c}^{k}\left(V \times S^{1}\right) \times \mathcal{H}\right)=C_{c}^{k}\left(V \times S^{1}\right) \times T_{w} \mathcal{H} \rightarrow \mathcal{E}_{w}
$$

is given for $\widehat{F} \in C_{c}^{k}\left(V \times S^{1}\right)$ and $\widehat{w} \in T_{w} \mathcal{H}$ by

$$
D S(F, w)(\widehat{F}, \widehat{w})=D s_{F}(w) \hat{w}+\left(\begin{array}{c}
-\nabla \hat{F}_{t}(v) \\
0
\end{array}\right) .
$$


We first show the following claim.

Claim For every $(F, w) \in S^{-1}(0)$ the operator $D S(F, w)$ is surjective.

Pick $(F, w) \in S^{-1}(0)$. Since $D s_{F}(w)$ is Fredholm, the image of $D S(F, w)$ is closed. Hence to show surjectivity, it suffices to prove that the orthogonal complement of the image of $D S(F, w)$ vanishes. To see that pick

$$
x=(y, \zeta) \in \operatorname{im} D S(F, w)^{\perp} .
$$

It follows from (31) that

$$
\left\{\begin{aligned}
\left\langle D s_{F}(w) \widehat{w}, x\right\rangle & =0, \quad \forall \widehat{w} \in T_{w} \mathcal{H}, \\
\langle\nabla \widehat{F}(v), y\rangle & =0, \quad \forall \widehat{F} \in C_{c}^{k}\left(V \times S^{1}\right) .
\end{aligned}\right.
$$

The first equation in (32) implies that

$$
x \in \operatorname{ker}\left(D s_{F}(w)\right)^{*}
$$

which forces $y$ to be of class $C^{k-1}$. Now we assume by contradiction that there exists $t_{0} \in S^{1}$ such that

$$
y\left(t_{0}\right) \neq 0
$$

Choose $\widehat{F}_{t_{0}} \in C_{c}^{k}(V)$ such that

$$
\left\langle\nabla \widehat{F}_{t_{0}}\left(v\left(t_{0}\right)\right), y\left(t_{0}\right)\right\rangle>0
$$

Since we have seen that $y$ is continuous, there exists $\epsilon>0$ such that

$$
\left\langle\nabla \widehat{F}_{t_{0}}(v(t)), y(t)\right\rangle \geq 0, \quad t \in\left(t_{0}-\epsilon, t_{0}+\epsilon\right) .
$$

Now choose a smooth cutoff function $\beta \in C^{\infty}\left(S^{1},[0,1]\right)$ such that $\beta\left(t_{0}\right)=1$ and $\beta(t)=0$ for $t \notin\left(t_{0}-\epsilon, t_{0}+\epsilon\right)$ and set $\widehat{F}_{t}=\beta(t) \widehat{F}_{t_{0}}$. It follows that

$$
\langle\nabla \hat{F}, y\rangle=\int_{0}^{1} \beta(t)\left\langle\nabla \widehat{F}_{t_{0}}(v(t)), y(t)\right\rangle d t>0,
$$

contradicting the second equation in (32). This proves that $y$ has to vanish identically. It remains to show that $\zeta$ vanishes. To see this we write

$$
\widehat{w}=(\widehat{v}, \widehat{\eta}) \in T_{w} \mathcal{H}=W^{1,2}\left(S^{1}, v^{*} T V\right) \times \mathbb{R} .
$$

Since $y$ vanishes identically the first equation in (32) becomes

$$
\zeta \int_{0}^{1} d H(v(t)) \hat{v}(t) d t=0, \quad \forall \hat{v} \in W^{1,2}\left(S^{1}, v^{*} T V\right) .
$$


Note that $(v, \eta)$ is a solution of (29). Hence, since 0 is a regular value of $H$, it follows from the second equation in (29) that $d H(v)$ does not vanish identically along $v$. Therefore, there exists $\hat{v} \in W^{1,2}\left(S^{1}, v^{*} T V\right)$ such that

$$
\int_{0}^{1} d H(v(t)) \widehat{v}(t) d t \neq 0
$$

Consequently,

$$
\zeta=0 .
$$

This finishes the proof of the claim.

Since $F \in C_{c}^{k}\left(V \times S^{1}\right)$ it follows that the section $S$ is $C^{k-1}$. Hence by the claim the implicit function theorem shows that $S^{-1}(0)$ is a $C^{k-1}$-manifold. Consider the $C^{k-1}-$ map

$$
p: S^{-1}(0) \rightarrow C_{c}^{k}\left(V \times S^{1}\right), \quad(F, w) \mapsto F .
$$

It follows from the Sard-Smale theorem that the set of regular values of the map $p$ is of the second category in $C_{c}^{k}\left(V \times S^{1}\right)$. But $F$ is a regular value of $p$, precisely if $s_{F} \pitchfork 0$. This finishes the proof of Step 1 .

Step 2 We prove the proposition.

We explain the argument by Taubes in our set-up (cf [53, page 52]).

Since $X_{H}$ and $X_{F_{t}}$ have compact support and 0 is a regular value of $H$ it follows that there exists a compact subset $V_{0} \subset V$ such that for every $(v, \eta) \in \operatorname{crit}\left(\mathcal{A}_{F}^{\alpha}\right)$,

$$
v(t) \in V_{0}, \quad t \in S^{1} .
$$

Choose $T>0$ and abbreviate

$$
\operatorname{crit}_{T}\left(\mathcal{A}_{F}^{\alpha}\right)=\left\{(v, \eta) \in \operatorname{crit}\left(\mathcal{A}_{F}^{\alpha}\right):|\eta| \leq T\right\} .
$$

It follows from (33), the first equation in (29) and the Theorem of Arzela-Ascoli that $\operatorname{crit}_{T}\left(\mathcal{A}_{F}^{\alpha}\right)$ is compact. For $2 \leq k \leq \infty$ we abbreviate

$$
\mathcal{U}_{T}^{k}(\alpha)=\left\{F \in C_{c}^{k}\left(V \times S^{1}\right): D s_{F}(w) \text { surjective, } \forall w \in \operatorname{crit}_{T}\left(\mathcal{A}_{F}^{\alpha}\right)\right\} .
$$

Since $\operatorname{crit}_{T}\left(\mathcal{A}_{F}^{\alpha}\right)$ is compact it follows that $\mathcal{U}_{T}^{k}(\alpha)$ is open in $C_{c}^{k}\left(V \times S^{1}\right)$. Moreover, it follows from Step 1 that if $k<\infty$ it is also dense in $C_{c}^{k}\left(V \times S^{1}\right)$. Since $C^{\infty}$ is dense in $C^{k}$ for every $k$, a diagonal argument shows that $\mathcal{U}_{T}^{\infty}(\alpha)$ is also dense in $C_{c}^{\infty}\left(V \times S^{1}\right)$. It follows that

$$
\mathcal{U}(\alpha)=\bigcap_{T \in \mathbb{N}} \mathcal{U}_{T}^{\infty}(\alpha)
$$

is of the second category in $C_{c}^{\infty}\left(V \times S^{1}\right)$. This finishes the proof of the proposition. 
If $J \in \mathcal{J}(\alpha)$ is an $\omega$-compatible almost complex structure and $m=m_{J}$ is the metric induced from $J$ we denote by $\mathcal{M}\left(\mathcal{A}_{F}^{\alpha}, J\right)$ the moduli space of all finite energy gradient flow lines of $\nabla_{m} \mathcal{A}_{F}^{\alpha}$. For $a, b \in \mathbb{R}$ we abbreviate

$$
\mathcal{M}_{a}^{b}\left(\mathcal{A}_{F}^{\alpha}, J\right)=\left\{w \in \mathcal{M}\left(\mathcal{A}_{F}^{\alpha}, J\right): \mathcal{A}_{F}^{\alpha}(w(s)) \in[a, b], \forall s \in \mathbb{R}\right\} .
$$

We cannot expect that gradient flow lines of the perturbed action functional are still compact up to breaking. However, we show that in the case of weakly tame stable hypersurfaces for small perturbations there is a decomposition of $\mathcal{M}_{a}^{b}\left(\mathcal{A}_{F}^{\alpha}, J\right)$ into a disjoint union of closed subsets

$$
\mathcal{M}_{a}^{b}\left(\mathcal{A}_{F}^{\alpha}, J\right)=\underline{\mathcal{M}}_{a}^{b}\left(\mathcal{A}_{F}^{\alpha}, J\right) \sqcup \overline{\mathcal{M}}_{a}^{b}\left(\mathcal{A}_{F}^{\alpha}, J\right)
$$

where $\underline{\mathcal{M}}_{a}^{b}\left(\mathcal{A}_{F}^{\alpha}, J\right)$ is compact up to breaking of gradient flow lines. We call $\underline{\mathcal{M}}_{a}^{b}\left(\mathcal{A}_{F}^{\alpha}, J\right)$ the essential part of the moduli space $\mathcal{M}_{a}^{b}\left(\mathcal{A}_{F}^{\alpha}, J\right)$ and $\overline{\mathcal{M}}_{a}^{b}\left(\mathcal{A}_{F}^{\alpha}, J ; a, b\right)$ the unessential part. The boundary operator then takes only account of the essential part of the moduli space of gradient flow lines.

If $\Sigma$ is weakly tame then by definition for $a \leq b$ the set

$$
\operatorname{crit}_{a}^{b}\left(\mathcal{A}^{\alpha}\right)=\left\{w \in \operatorname{crit}\left(\mathcal{A}^{\alpha}\right): a \leq \mathcal{A}^{\alpha}(w) \leq b\right\}
$$

is compact. Hence we can define

$$
\begin{aligned}
& \aleph(a, b ; \alpha)=\min \left\{a, \min \left\{\mathcal{A}(w): w \in \operatorname{crit}_{a}^{b}\left(\mathcal{A}^{\alpha}\right)\right\}\right\}, \\
& \beth(a, b ; \alpha)=\max \left\{b, \max \left\{\mathcal{A}^{\alpha}(w): w \in \operatorname{crit}_{a}^{b}\left(\mathcal{A}^{\alpha}\right)\right\}\right\} .
\end{aligned}
$$

Note that $\aleph(a, b)$ and $\beth(a, b)$ actually only depend on $\pi(\alpha)=\lambda \in \Lambda_{S}(\Sigma, \omega)$ so that we can set

$$
\aleph(a, b ; \lambda)=\aleph(a, b ; \alpha), \quad \beth(a, b ; \lambda)=\beth(a, b ; \alpha) .
$$

We next introduce a subspace of perturbations $F$ which have the property that the Lagrange multiplier along gradient flow lines either becomes large or remains small. We first introduce the number

$$
\jmath(a, b ; \lambda)=\left(\frac{5}{2}\right)^{2}(\beth(a, b ; \lambda)-\aleph(a, b ; \lambda))
$$

and define the interval

$$
I(a, b ; \lambda)=[\beth(a, b ; \lambda)+3, \jmath(a, b ; \lambda)+4] .
$$

We set

$$
\tilde{\mathcal{U}}_{a}^{b}(\alpha, J)=\left\{F \in C_{c}^{\infty}\left(V \times S^{1}\right):\|\eta\|_{\infty} \notin I(a, b ; \lambda), \forall w=(v, \eta) \in \mathcal{M}_{a}^{b}\left(\mathcal{A}_{F}^{\alpha}, J\right)\right\} .
$$


Lemma 4.12 Assume that $\Sigma$ is a weakly tame stable hypersurface in a symplectically aspherical, geometrically bounded, symplectic manifold $(V, \omega)$ and $\alpha \in \mathfrak{A}(\Sigma, V)$ is a $(3 / 5, \epsilon)$-admissible quintuple with $\epsilon \leq 1 / \mathcal{J}(a, b ; \lambda), J \in \mathcal{J}(\alpha)$ and $a \leq b$. Then $\tilde{\mathcal{U}}_{a}^{b}(\alpha, J)$ is open and nonempty.

Proof We first show that $\tilde{\mathcal{U}}_{a}^{b}(\alpha, J)$ is open. This is actually true for any admissible quintuple $\alpha$. For openness we prove that the complement is closed. Hence let

$$
F_{v} \in C_{c}^{\infty}\left(V \times S^{1}\right) \backslash \tilde{\mathcal{U}}_{a}^{b}(\alpha, J), \quad v \in \mathbb{N}
$$

be a sequence of perturbations in the complement of $\tilde{\mathcal{U}}_{a}^{b}(\alpha, J)$ such that

$$
\lim _{\nu \rightarrow \infty} F_{\nu}=F \in C^{\infty}\left(V \times S^{1}\right) .
$$

It remains to show that $F \notin \tilde{\mathcal{U}}_{a}^{b}(\alpha, J)$. By definition of $\tilde{\mathcal{U}}_{a}^{b}(\alpha, J)$ there exists for each $v \in \mathbb{N}$ a gradient flow line

$$
w_{\nu}=\left(v_{\nu}, \eta_{v}\right) \in \mathcal{M}_{a}^{b}\left(\mathcal{A}_{F_{v}}^{\alpha}, J\right)
$$

such that

$$
\left\|\eta_{\nu}\right\|_{\infty} \in I(a, b ; \lambda)
$$

Since the Lagrange multiplier is uniformly bounded the usual compactness arguments in Floer homology (boundedness at infinity and no bubbling because of symplectic asphericity) imply that $w_{v}$ has a convergent subsequence $w_{v_{j}}$ such that

$$
\lim _{j \rightarrow \infty} w_{v_{j}}=w=(v, \eta) \in \mathcal{M}_{a}^{b}\left(\mathcal{A}_{F}^{\alpha}, J\right)
$$

Since $I(a, b ; \lambda)$ is closed it follows from (34) that

Consequently

$$
\|\eta\|_{\infty} \in I(a, b ; \lambda) .
$$

This finishes the proof of openness.

To see finally that $\tilde{\mathcal{U}}_{a}^{b}(\alpha, J)$ is nonempty we observe that by our assumptions on the admissible quintuple $\alpha$ it follows from Corollary 4.5 that

$$
0 \in \tilde{\mathcal{U}}_{a}^{b}(\alpha, J) .
$$

Hence we are done with the proof of the Lemma. 
Definition For a fixed pair $(\alpha, J)$ consisting of an admissible quintuple $\alpha$ and an $\omega$-compatible almost complex structure $J \in \mathcal{J}(\alpha)$ and $a \leq b$ we say that a perturbation $F \in C_{c}^{\infty}\left(V \times S^{1}\right)$ is $(a, b)$-approvable if $\mathcal{A}_{F}^{\alpha}$ is Morse and contained in $\tilde{\mathcal{U}}_{a}^{b}(\alpha, J)$. We set

$$
\mathcal{U}_{a}^{b}(\alpha, J)=\left\{F \in \tilde{\mathcal{U}}_{a}^{b}(\alpha, J): \mathcal{A}_{F}^{\alpha} \text { Morse }\right\}
$$

for the set of $(a, b)$-approvable perturbations.

Combining Proposition 4.11 with Lemma 4.12 we obtain the following Corollary.

Corollary 4.13 Under the assumptions of Lemma 4.12 the set $\mathcal{U}_{a}^{b}(\alpha, J)$ is nonempty.

We finally explain how to associate to an $(a, b)$-approvable perturbation $F$ a homology group $H F_{a}^{b}\left(\mathcal{A}_{F}^{\alpha}, J\right)$. We first define the set

$$
\mathcal{C}_{a}^{b}\left(\mathcal{A}_{F}^{\alpha}\right)=\left\{w=(v, \eta) \in \operatorname{crit}_{a}^{b}\left(\mathcal{A}_{F}^{\alpha}\right):|\eta|<\mathfrak{I}(a, b ; \lambda)+3\right\} .
$$

Because $\operatorname{crit}_{a}^{b}\left(\mathcal{A}_{F}^{\alpha}\right) \subset \mathcal{M}_{a}^{b}\left(\mathcal{A}_{F}^{\alpha}, J\right)$ and $F$ is $(a, b)$-approvable we infer

$$
\mathcal{C}_{a}^{b}\left(\mathcal{A}_{F}^{\alpha}\right)=\left\{w=(v, \eta) \in \operatorname{crit}_{a}^{b}\left(\mathcal{A}_{F}^{\alpha}\right):|\eta| \leq \mathfrak{I}(a, b ; \lambda)+3\right\} .
$$

Applying the Theorem of Arzela-Ascoli to the critical point equation (29) of $\mathcal{A}_{F}^{\alpha}$ we see that $\mathcal{C}_{a}^{b}\left(\mathcal{A}_{F}^{\alpha}\right)$ is compact. Since $\mathcal{A}_{F}^{\alpha}$ is Morse, the set $\mathcal{C}_{a}^{b}\left(\mathcal{A}_{F}^{\alpha}\right)$ is also discrete and hence finite. Thus

$$
C F_{a}^{b}\left(\mathcal{A}_{F}^{\alpha}\right)=\mathcal{C}_{a}^{b}\left(\mathcal{A}_{F}^{\alpha}\right) \otimes \mathbb{Z}_{2}
$$

is a finite dimensional $\mathbb{Z}_{2}$-vector space. We further define the essential part of the moduli space $\mathcal{M}_{a}^{b}\left(\mathcal{A}_{F}^{\alpha}, J\right)$ to be

$$
\underline{\mathcal{M}}_{a}^{b}\left(\mathcal{A}_{F}^{\alpha}, J\right)=\left\{w=(v, \eta) \in \mathcal{M}_{a}^{b}\left(\mathcal{A}_{F}^{\alpha}, J\right):\|\eta\|_{\infty}<\mathfrak{J}(a, b ; \lambda)+3\right\} .
$$

Since $F$ is $(a, b)$-approvable, $\mathcal{M}_{a}^{b}\left(\mathcal{A}_{F}^{\alpha}, J\right)$ is a closed subset of $\mathcal{M}_{a}^{b}\left(\mathcal{A}_{F}^{\alpha}, J\right)$. Moreover, since the Lagrange multiplier is by definition uniformly bounded in $\underline{\mathcal{M}}_{a}^{b}\left(\mathcal{A}_{F}^{\alpha}, J\right)$ standard arguments in Floer theory using that $(V, \omega)$ is geometrically bounded and symplectically aspherical imply that $\underline{\mathcal{M}}_{a}^{b}\left(\mathcal{A}_{F}^{\alpha}, J\right)$ is $C_{\text {loc }}^{\infty}$-compact. Hence we can use $\underline{\mathcal{M}}_{a}^{b}\left(\mathcal{A}_{F}^{\alpha}, J\right)$ to define a linear map

$$
\partial_{a}^{b}: C F_{a}^{b}\left(\mathcal{A}_{F}^{\alpha}\right) \rightarrow C F_{a}^{b}\left(\mathcal{A}_{F}^{\alpha}\right)
$$

by counting gradient flow lines. Since the metric $m$ induced from $J$ does not necessarily fulfill the Morse-Smale condition one may need to perturb the gradient flow equation to show that $\partial_{a}^{b} \circ \partial_{a}^{b}=0$. It is quite likely that this can actually be achieved by taking a generic family of $\omega$-compatible almost complex structures $J_{t}$. However, we have not checked the details since this is nowadays not needed anymore in view of the progress 
of abstract perturbation theory. For example, if one compactifies the moduli space of unparametrised trajectories $\underline{\mathcal{M}}_{a}^{b}\left(\mathcal{A}_{F}^{\alpha}, J\right) / \mathbb{R}$ by adding broken gradient flow lines, this compactified space can be interpreted as the zero-set of a Fredholm-section

$$
\varsigma: \mathcal{P} \rightarrow \mathcal{E}
$$

where $\mathcal{P}$ is an $M$-polyfold and $\mathcal{E}$ is an $M$-polyfold bundle over $\mathcal{P}$ [38]. If one perturbs this section to make it transverse [39], one can define a boundary operator by counting the perturbed broken gradient trajectories between two critical points. Indeed, if $\wp$ is a generic abstract perturbation and

$$
\varsigma_{\wp}: \mathcal{P} \rightarrow \mathcal{E}
$$

is the perturbed section, then its zero-set

$$
\mathcal{N}(\wp)=\varsigma_{\wp}^{-1}(0)
$$

is a compact manifold with corners. We denote by $\mathcal{N}_{0}(\wp)$ its zero-dimensional part. For a generic perturbation the section $\varsigma_{\wp}$ is also transverse with respect to the boundary of the polyfold $\mathcal{P}$ and hence elements in $\mathcal{N}_{0}(\wp)$ consist still of unparametrised trajectories $[w] \in C^{\infty}(\mathbb{R}, \mathcal{L} \times \mathbb{R}) / \mathbb{R}$ which are unbroken and converge asymptotically to critical points in $\mathcal{C}_{a}^{b}\left(\mathcal{A}_{F}^{\alpha}\right)$. Hence for $w^{ \pm} \in \mathcal{C}_{a}^{b}\left(\mathcal{A}_{F}^{\alpha}\right)$ we abbreviate

$$
\mathcal{N}_{0}\left(\wp ; w^{-}, w^{+}\right)=\left\{[w] \in \mathcal{N}_{0}(\wp): \lim _{s \rightarrow \pm \infty} w(s)=w^{ \pm}\right\}
$$

and introduce the $\mathbb{Z}_{2}$-number

$$
n\left(w^{-}, w^{+}\right)=\#_{2} \mathcal{N}_{0}\left(\wp ; w^{-}, w^{+}\right)
$$

where $\#_{2}$ denotes the count in $\mathbb{Z}_{2}$. We define a linear map

$$
\partial_{a}^{b}\left(\mathcal{A}_{F}^{\alpha}, J ; \wp\right): C F_{a}^{b}\left(\mathcal{A}_{F}^{\alpha}\right) \rightarrow C F_{a}^{b}\left(\mathcal{A}_{F}^{\alpha}\right)
$$

which is defined on generators by

$$
\partial_{a}^{b}\left(\mathcal{A}_{F}^{\alpha}, J ; \wp\right)\left[w^{+}\right]=\sum_{w^{-} \in \mathcal{C}_{a}^{b}\left(\mathcal{A}_{F}^{\alpha}\right)} n\left(w^{-}, w^{+}\right) w^{-} .
$$

Standard arguments show that $\partial_{a}^{b}\left(\mathcal{A}_{F}^{\alpha}, J ; \wp\right)$ is actually a boundary operator, ie its square is zero. The boundary operator might depend indeed on the abstract perturbation but the homology

$$
H F_{a}^{b}\left(\mathcal{A}_{F}^{\alpha}, J\right)=\frac{\operatorname{ker} \partial_{a}^{b}\left(\mathcal{A}_{F}^{\alpha}, J ; \wp\right)}{\operatorname{im} \partial_{a}^{b}\left(\mathcal{A}_{F}^{\alpha}, J ; \wp\right)}
$$

is independent of the abstract perturbation by standard homotopy arguments. 


\subsection{Definition of Rabinowitz Floer homology}

In this subsection we assume that $\Sigma$ is a weakly tame stable hypersurface in a symplectically aspherical, geometrically bounded, symplectic manifold $(V, \omega)$. In this situation we define Rabinowitz Floer homology. We further compute it for two relevant cases. One case is when there are no closed orbits in $\Sigma$ contractible in $V$, and the other is when $\Sigma$ is displaceable.

We define the $\Omega$-spectrum of $\Sigma$ to be

$$
\mho(\Sigma)=\{ \pm \Omega(v): v \in X(\Sigma)\} \cup\{0\}
$$

where we recall that $X(\Sigma)$ denotes the set of closed characteristics in $\Sigma$ which are contractible in $V$. Since each $\Omega(v)$ corresponds to a critical value of Rabinowitz action functional $\mathcal{A}^{\alpha}$ for an admissible quintuple $\alpha \in \mathfrak{A}(\Sigma, V)$ the set $\mho(\Sigma)$ is a meager subset of $\mathbb{R}$ by Sard's theorem.

Our first aim is to define Rabinowitz Floer homology groups $R F H_{a}^{b}$ for $a, b \notin \mho(\Sigma)$. These groups basically depend only on $\Sigma$ and $V$. However, there is a little subtlety to note. We do not know if the space of all $\omega$-compatible geometrically bounded almost complex structures $J$ on $V$ is connected. Therefore Rabinowitz Floer homology actually could depend on the choice of the geometrically bounded compatible almost complex structure. We therefore fix one such complex structure $J_{0}$. For an admissible quintuple $\alpha$ we abbreviate by

$$
\mathcal{J}\left(\alpha, J_{0}\right) \subset \mathcal{J}(\alpha)
$$

the set of all $J \in \mathcal{J}(\alpha)$ which outside of a compact set coincide with $J_{0}$. We observe that the space $\mathcal{J}\left(\alpha, J_{0}\right)$ is connected. It is possible that Rabinowitz Floer homology depends on the choice of $J_{0}$, although this seems unlikely. We refer in the following to the triple $\left(V, \omega, J_{0}\right)$ as the geometrically bounded symplectic manifold. We often skip the reference to $\omega$ and $J_{0}$ and just mention $V$.

If $a, b \notin \mho(\Sigma), \alpha \in \mathfrak{A}(\Sigma, V)$ meets the assumptions of Lemma 4.12, and $J \in \mathcal{J}\left(\alpha, J_{0}\right)$ we introduce the subset of perturbations

$$
\tilde{\mathcal{V}}_{a}^{b}(\alpha, J)=\left\{F \in \tilde{\mathcal{U}}_{a}^{b}(\alpha, J): \mathcal{A}_{F}^{\alpha}(w) \notin\{a, b\}, \forall w \in \mathcal{C}_{a}^{b}\left(\mathcal{A}_{F}^{\alpha}, J\right)\right\}
$$

where we refer to (35) for the definition of $\mathcal{C}_{a}^{b}\left(\mathcal{A}_{F}^{\alpha}, J\right)$. By the Theorem of ArzelaAscoli $\tilde{\mathcal{V}}_{a}^{b}(\alpha, J)$ is an open subset of $\tilde{\mathcal{U}}_{a}^{b}(\alpha, J)$. Moreover, since $a, b \notin \mho(\Sigma)$ the zero perturbation is contained in $\tilde{\mathcal{V}}_{a}^{b}(\alpha, J)$. Hence we abbreviate by

$$
\widehat{\mathcal{V}}_{a}^{b}(\alpha, J) \subset \tilde{\mathcal{V}}_{a}^{b}(\alpha, J)
$$


the connected component of $\tilde{\mathcal{V}}(\alpha, J)$ containing 0 . We set

$$
\mathcal{V}_{a}^{b}(\alpha, J)=\left\{F \in \hat{\mathcal{V}}_{a}^{b}(\alpha, J): \mathcal{A}_{F}^{\alpha} \text { Morse }\right\} .
$$

By Proposition 4.11 and Lemma 4.12 the set $\mathcal{V}_{a}^{b}(\alpha, J)$ is nonempty. Hence we pick $F \in \mathcal{V}_{a}^{b}(\alpha, J)$ and define

$$
R F H_{a}^{b}(\Sigma, V)=H F_{a}^{b}\left(\mathcal{A}_{F}^{\alpha}, J\right)
$$

where the right-hand side was defined in (36). It is straightforward to check that this definition is independent of the choices of $F, J$ and $\alpha$. For this we actually use that the space $\mathcal{J}\left(\alpha, J_{0}\right)$ is connected.

There are canonical homomorphisms between Rabinowitz Floer homology groups

and

$$
\begin{aligned}
\pi_{a_{2}, a_{1}}^{b}: R F H_{a_{1}}^{b} \rightarrow R F H_{a_{2}}^{b}, & a_{1} \leq a_{2} \leq b \\
\iota_{a}^{b_{2}, b_{1}}: R F H_{a}^{b_{1}} \rightarrow R F H_{a}^{b_{2}}, & a \leq b_{1} \leq b_{2} .
\end{aligned}
$$

These maps satisfy

$$
\left\{\begin{aligned}
\pi_{a_{3}, a_{2}}^{b} \circ \pi_{a_{2}, a_{1}}^{b} & =\pi_{a_{3}, a_{1},}^{b}, & & a_{1} \leq a_{2} \leq a_{3} \leq b, \\
\iota_{a}^{b_{3}, b_{2}} \circ \iota_{a}^{b_{2}, b_{1}} & =\iota_{a}^{b_{3}, b_{1}}, & & a \leq b_{1} \leq b_{2} \leq b_{3}, \\
\iota_{a_{2}}^{b_{2}, b_{1}} \circ \pi_{a_{2}, a_{1}}^{b_{1}} & =\pi_{a_{2}, a_{1}}^{b_{2}} \circ \iota_{a_{1}}^{b_{2}, b_{1}}, & & a_{1} \leq a_{2} \leq b_{1} \leq b_{2} .
\end{aligned}\right.
$$

In particular, the Rabinowitz Floer homology groups together with these maps have the structure of a bidirect system of $\mathbb{Z}_{2}$-vector spaces.

We next describe the construction of the map $\pi_{a_{2}, a_{1}}^{b}$. Assume that

$$
a_{1} \leq a_{2} \leq b \quad a_{1}, a_{2}, b \notin \mho(\Sigma) .
$$

We first pick a small stabilizing 1 -form $\lambda \in \Lambda_{S}(\Sigma, V)$. We note that

$$
\mathfrak{I}\left(a_{1}, b ; \lambda\right) \geq \mathfrak{I}\left(a_{2}, b ; \lambda\right) .
$$

We pick a $(3 / 5, \epsilon)$-admissible quintuple $\alpha$ with $\pi(\alpha)=\lambda$ and $\epsilon \leq 1 / J\left(a_{1}, b ; \lambda\right)$. Note that because of (38) this quintuple meets the assumptions of Lemma 4.12 for $\left(a_{1}, b\right)$ as well as for $\left(a_{2}, b\right)$. We introduce the closed interval

$$
I\left(a_{1}, a_{2}, b ; \lambda\right)=\left[\exists\left(a_{2}, b ; \lambda\right)+3, \jmath\left(a_{1}, b ; \lambda\right)+4\right] \supset I\left(a_{2}, b ; \lambda\right) .
$$

We set

$\tilde{\mathcal{U}}_{a_{2}, a_{1}}^{b}(\alpha, J)=\left\{F \in \tilde{\mathcal{U}}_{a_{1}}^{b}(\alpha, J):\|\eta\|_{\infty} \notin I\left(a_{1}, a_{2}, b ; \lambda\right), \forall(v, \eta) \in \mathcal{M}_{a_{2}}^{b}\left(\mathcal{A}_{F}^{\alpha}, J\right)\right\}$. 
A similar reasoning as in Lemma 4.12 shows that $\tilde{\mathcal{U}}_{a_{2}, a_{1}}^{b}(\alpha, J)$ is an open set of $C_{c}^{\infty}\left(V \times S^{1}\right)$ containing the zero perturbation. We define

$$
\tilde{\mathcal{V}}_{a_{2}, a_{1}}^{b}(\alpha, J)=\left\{F \in \tilde{\mathcal{U}}_{a_{2}, a_{1}}^{b}(\alpha, J): \mathcal{A}_{F}^{\alpha}(w) \notin\left\{a_{1}, a_{2}, b\right\}, \forall w \in \mathcal{C}_{a_{1}}^{b}\left(\mathcal{A}_{F}^{\alpha}, J\right)\right\} .
$$

Again this is an open subset of $C_{c}^{\infty}\left(V \times S^{1}\right)$ containing the zero perturbation. Let

$$
\hat{\mathcal{V}}_{a_{2}, a_{1}}^{b}(\alpha, J) \subset \tilde{\mathcal{V}}_{a_{2}, a_{1}}^{b}(\alpha, J)
$$

be the connected component containing 0 and set

$$
\mathcal{V}_{a_{2}, a_{1}}^{b}(\alpha, J)=\left\{F \in \hat{\mathcal{V}}_{a_{2}, a_{1}}^{b}(\alpha, J): \mathcal{A}_{F}^{\alpha} \text { Morse }\right\} .
$$

Note that $\mathcal{V}_{a_{2}, a_{1}}^{b}(\alpha, J)$ is nonempty and satisfies

$$
\mathcal{V}_{a_{2}, a_{1}}^{b}(\alpha, J) \subset \mathcal{V}_{a_{1}}^{b}(\alpha, J) \cap \mathcal{V}_{a_{2}}^{b}(\alpha, J) .
$$

We pick

$$
F \in \mathcal{V}_{a_{2}, a_{1}}^{b}(\alpha, J) \text {. }
$$

Abbreviate

$$
\mathcal{C}_{a_{1}}^{a_{2}}\left(\mathcal{A}_{F}^{\alpha}, b\right)=\left\{w=(v, \eta) \in \operatorname{crit}_{a_{1}}^{a_{2}}\left(\mathcal{A}_{F}^{\alpha}\right):|\eta|<\mathfrak{I}\left(a_{1}, b ; \lambda\right)+3\right\} .
$$

Since $a_{2}$ is not a critical value of $\mathcal{A}_{F}^{\alpha}$ and there is no critical point $w=(v, \eta) \in$ crit $_{a_{2}}^{b}\left(\mathcal{A}_{F}^{\alpha}\right) \subset \mathcal{M}_{a_{2}}^{b}\left(\mathcal{A}_{F}^{\alpha}, J\right)$ with $\eta \in I\left(a_{1}, a_{2}, b ; \lambda\right)$, we have a disjoint union

$$
\mathcal{C}_{a_{1}}^{b}\left(\mathcal{A}_{F}^{\alpha}\right)=\mathcal{C}_{a_{2}}^{b}\left(\mathcal{A}_{F}^{\alpha}\right) \sqcup \mathcal{C}_{a_{1}}^{a_{2}}\left(\mathcal{A}_{F}^{\alpha}, b\right) .
$$

This leads to a direct sum of $\mathbb{Z}_{2}$-vector spaces

$$
C F_{a_{1}}^{b}\left(\mathcal{A}_{F}^{\alpha}\right)=C F_{a_{2}}^{b}\left(\mathcal{A}_{F}^{\alpha}\right) \oplus C F_{a_{1}}^{a_{2}}\left(\mathcal{A}_{F}^{\alpha}, b\right)
$$

with

$$
C F_{a_{1}}^{a_{2}}\left(\mathcal{A}_{F}^{\alpha}, b\right)=\mathcal{C}_{a_{1}}^{a_{2}}\left(\mathcal{A}_{F}^{\alpha}, b\right) \otimes \mathbb{Z}_{2} \text {. }
$$

Let

$$
p_{a_{2}, a_{1}}^{b}: C F_{a_{1}}^{b}\left(\mathcal{A}_{F}^{\alpha}\right) \rightarrow C F_{a_{2}}^{b}\left(\mathcal{A}_{F}^{\alpha}\right)
$$

be the projection along $C F_{a_{1}}^{a_{2}}\left(\mathcal{A}_{F}^{\alpha}, b\right)$. Using the fact that the action is increasing along gradient flow lines we observe that the projection $p_{a_{2}, a_{1}}^{b}$ commutes with the boundary operators and hence induces a homomorphism

$$
\pi_{a_{2}, a_{1}}^{b}: H F_{a_{1}}^{b}\left(\mathcal{A}_{F}^{\alpha}, J\right) \rightarrow H F_{a_{2}}^{b}\left(\mathcal{A}_{F}^{\alpha}, J\right) .
$$

A usual homotopy argument shows that this homomorphism is independent of $F, \alpha$, and $J$ and hence can be interpreted as homomorphism

$$
\pi_{a_{2}, a_{1}}^{b}: R F H_{a_{1}}^{b} \rightarrow R F H_{a_{2}}^{b} .
$$


The construction of the homomorphism

$$
\iota_{a}^{b_{2}, b_{1}}: R F H_{a}^{b_{1}} \rightarrow R F H_{a}^{b_{1}}
$$

is similar and will not be carried out here. We also omit the proof of (37).

Given the bidirect system of $\mathbb{Z}_{2}$-vector spaces $(R F H, \pi, \iota)$ we can extract out of it two Rabinowitz Floer homology groups

$$
\begin{aligned}
& \overline{R F H}=\underset{b \rightarrow \infty}{\lim _{a \rightarrow-\infty}} \lim _{a \rightarrow} R F H_{a}^{b}, \\
& \underline{R F H}={\underset{a \rightarrow-\infty}{\lim } \underset{b \rightarrow \infty}{\lim } R F H_{a}^{b} .}^{\lim _{b \rightarrow \infty}}
\end{aligned}
$$

Since inverse and direct limits do not necessarily commute it is an open problem if the two Rabinowitz Floer homology groups coincide. However, it is well known (see Mac Lane [47, page 215]) that there is a canonical homomorphism which takes account of the interchange of inverse and direct limits

$$
\kappa: \overline{R F H} \rightarrow \underline{R F H} .
$$

In general the canonical homomorphism does not need to be an isomorphism, although we have no example where $\kappa$ fails to be an isomorphism in Rabinowitz Floer homology. We finish this subsection by computing the Rabinowitz Floer homology groups in two easy examples.

Theorem 4.14 Suppose that $\Sigma$ is a weakly tame, stable hypersurface in a symplectically aspherical, geometrically bounded, symplectic manifold $(V, \omega)$.

(i) Assume that there are no closed characteristics in $\Sigma$ which are contractible in $V$. Then

$$
\overline{R F H}(\Sigma, V)=\underline{R F H}(\Sigma, V)=H\left(\Sigma ; \mathbb{Z}_{2}\right),
$$

where $H$ denotes (ungraded) singular homology.

(ii) Assume that $\Sigma$ is displaceable in $V$. Then

$$
\overline{R F H}(\Sigma, V)=\underline{R F H}(\Sigma, V)=\{0\} .
$$

Proof We first prove assertion (i). If there are no closed characteristics on $\Sigma$ which are contractible in $V$, the unperturbed Rabinowitz action functional $\mathcal{A}^{\alpha}$ is Morse-Bott with critical manifold given by $\{(x, 0)\}$ where $x \in \Sigma$ is thought of as a constant loop. Since $\mathcal{A}^{\alpha}$ is constant on the critical manifold its homology is just the homology of the critical manifold, hence the homology of the hypersurface $\Sigma$. 
We next show assertion (ii). A reasoning similar as in the proof of Theorem 4.9 shows that if $d \geq e(\Sigma)$, the displacement energy of $\Sigma$, and $a \leq b$, then

$$
\pi_{a+d, a}^{b+d} \circ \iota_{a}^{b+d, b}=0: R F H_{a}^{b}(\Sigma, V) \rightarrow R F H_{a+d}^{b+d}(\Sigma, V) .
$$

This implies the vanishing of the two Rabinowitz Floer homology groups.

\subsection{Invariance}

In this subsection we show that Rabinowitz Floer homology is invariant under stable tame homotopies.

Theorem 4.15 Assume that $\mathcal{S}=\left(\Sigma_{\zeta}, \lambda_{\zeta}\right)$ for $\zeta \in[0,1]$ is a stable tame homotopy. Then there are isomorphisms

$$
\begin{aligned}
& \bar{\Phi}=\bar{\Phi}_{\mathcal{S}}: \overline{R F H}\left(\Sigma_{0}, V\right) \rightarrow \overline{R F H}\left(\Sigma_{1}, V\right), \\
& \underline{\Phi}=\underline{\Phi}^{\mathcal{S}}: \underline{\operatorname{RFH}}\left(\Sigma_{0}, V\right) \rightarrow \underline{\operatorname{RFH}}\left(\Sigma_{1}, V\right),
\end{aligned}
$$

such that the following diagram commutes:

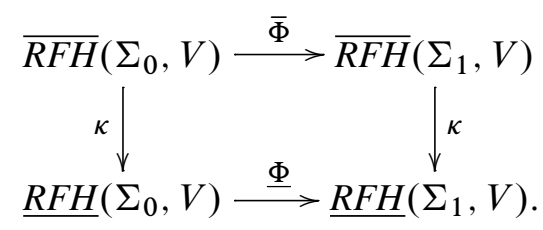

Proof Given a stable homotopy $\mathcal{S}=\left(\Sigma_{\zeta}, \lambda_{\zeta}\right)$ there exists a smooth family of positive real numbers $\sigma_{\zeta}>0$, such that $\sigma_{\zeta} \lambda_{\zeta}$ is small for every $\zeta \in[0,1]$ and there exists a smooth path $\tau_{\zeta} \in \mathcal{T}_{\ell}\left(\Sigma_{\zeta}, \sigma_{\zeta} \lambda_{\zeta}\right)$ of large tubular neighbourhoods and a smooth path $I_{\zeta} \in \mathcal{I}_{s}\left(\Sigma_{\zeta}, \lambda_{\zeta}\right)$ of small $\omega_{\Sigma_{\zeta}}$-compatible complex structures such that

$$
\mathfrak{P}=\left(\Sigma_{\zeta}, \sigma_{\zeta} \lambda_{\zeta}, \tau_{\zeta}, I_{\zeta}\right)
$$

is a smooth path of stable quadruples. We further remark that the stable homotopy $\left(\Sigma_{\zeta}, \sigma_{\zeta} \lambda_{\zeta}\right)$ is still tame.

Since each path of stable quadruples can be obtained by concatenating short paths it actually suffices to prove the theorem only for short paths of stable quadruples. We need the following Lemma. We refer the reader to (14) for the definition of $\mathcal{H}\left(\alpha^{-}, \alpha^{+}\right)$ and to Theorem 4.8 for the definition of $\Delta(1 / 2)$. 
Lemma 4.16 Assume that $\mathfrak{P}=\left(\Sigma_{\zeta}, \lambda_{\zeta}, \tau_{\zeta}, I_{\zeta}\right)$ for $\zeta \in[0,1]$ is a short path of stable quadruples such that $\left(\Sigma_{\zeta}, \lambda_{\zeta}\right)$ is tame with taming constant $c$ and $\mathfrak{P}$ is short in the sense that

$$
\Delta(\mathfrak{P}) \leq \min \left\{\frac{1}{144 \cdot \max \{1, c\}}, \Delta\left(\frac{1}{2}\right)\right\} .
$$

Then there exist admissible quintuples $\alpha^{-}$for $\Sigma_{0}$ and $\alpha^{+}$for $\Sigma_{1}$, a time dependent Hamiltonian $H \in \mathcal{H}\left(\alpha^{-}, \alpha^{+}\right)$, and a time-dependent metric $m=\left\{m_{s}\right\}_{s \in \mathbb{R}}$ on $\mathcal{L} \times \mathbb{R}$ which is constant for $|s|$ large such that the following holds true. Suppose that $w=$ $(v, \eta) \in C^{\infty}(\mathbb{R}, \mathcal{L} \times \mathbb{R})$ is a gradient flow line of the time dependent gradient $\nabla_{m_{s}} \mathcal{A}^{H_{s}}$ which converges asymptotically $\lim _{s \rightarrow \pm \infty} w(s)=w^{ \pm}$to critical points of $\mathcal{A}^{\alpha^{ \pm}}$, such that $a=\mathcal{A}^{\alpha^{-}}\left(w^{-}\right)$and $b=\mathcal{A}^{\alpha^{+}}\left(w^{+}\right)$. Then the following holds:

(i) If $a \geq 1 / 9$, then $b \geq a / 2$.

(ii) If $b \leq-1 / 9$, then $a \leq b / 2$.

Proof We choose $H$ as in Theorem 4.8. Combining tameness with Theorem 4.8 for $\epsilon=1 / 2$ and using (40) we obtain

$$
\|\eta\|_{\infty} \leq 9 \max \{c, 1\}|b-a|+2 .
$$

Moreover, by letting $\sigma$ tend to $\infty$ in Step 1 of Theorem 4.8 we obtain

$$
b \geq a-2 \Delta(\mathfrak{P})\|\eta\|_{\infty} .
$$

From (40)-(42) we extract

$$
b \geq a-\frac{|b-a|}{8}-\frac{1}{36} .
$$

To prove assertion (i) we first consider the case

$$
|b| \leq a, \quad a \geq \frac{1}{9} .
$$

In this case we estimate

$$
b \geq a-\frac{|a|}{4}-\frac{1}{36}=\frac{3 a}{4}-\frac{1}{36} \geq \frac{a}{2} .
$$

Hence to prove assertion (i) it suffices to exclude the case

$$
-b \geq a \geq \frac{1}{9} .
$$

But in this case (43) leads to a contradiction in the following way:

$$
b \geq \frac{1}{9}-\frac{|b-a|}{8}-\frac{1}{36} \geq-\frac{|b-a|}{8} \geq \frac{b}{4}
$$


implying that $3 b / 4 \geq 0$ and hence $b \geq 0$ contradicting (44). This proves the first assertion.

To prove assertion (ii) we set

$$
b^{\prime}=-a, \quad a^{\prime}=-b .
$$

We note that if (43) holds for $a$ and $b$, it also holds for $b^{\prime}$ and $a^{\prime}$. Hence we get from assertion (i) the implication

$$
-b \geq \frac{1}{9} \quad \Rightarrow \quad-a \geq-\frac{b}{2}
$$

which is equivalent to assertion (ii). This finishes the proof of the Lemma.

Proof of Theorem 4.15 continued In view of Lemma 4.16 we obtain for $a \leq-1 / 9$ and $b \geq 1 / 9$ homomorphisms

$$
\Phi_{a}^{b}: R F H_{a}^{b / 2}\left(\Sigma_{0}, V\right) \rightarrow R F H_{a / 2}^{b}\left(\Sigma_{1}, V\right)
$$

defined by counting gradient flow lines of the time dependent Rabinowitz action functional. Again to count gradient flow lines we have to choose small perturbations which make Rabinowitz action functional Morse and then take the count (possibly after a further abstract perturbation) of the essential part of the moduli space of gradient flow lines of the perturbed time dependent functional. These homomorphisms interchange the maps $\pi$ and $\iota$ and hence induce homomorphisms

$$
\begin{aligned}
& \bar{\Phi}: \overline{R F H}\left(\Sigma_{0}, V\right) \rightarrow \overline{R F H}\left(\Sigma_{1}, V\right), \\
& \underline{\Phi}: \underline{\operatorname{RFH}}\left(\Sigma_{0}, V\right) \rightarrow \underline{\operatorname{RFH}}\left(\Sigma_{1}, V\right),
\end{aligned}
$$

such that (39) commutes.

It remains to show that $\bar{\Phi}$ and $\underline{\Phi}$ are isomorphisms. By using the homotopy backwards we get homomorphisms

$$
\Psi_{a}^{b}: R F H_{a}^{b / 2}\left(\Sigma_{1}, V\right) \rightarrow R F H_{a / 2}^{b}\left(\Sigma_{0}, V\right) .
$$

A standard homotopy of homotopy argument shows that for $a \leq-2 / 9$ and $b \geq 2 / 9$ the composition of $\Phi$ and $\Psi$ is given by

$$
\Psi_{a / 2}^{b} \circ \Phi_{a}^{b / 2}=\pi_{a / 4, a}^{b} \circ \iota_{a}^{b, b / 4}: R F H_{a}^{b / 4}\left(\Sigma_{0}, V\right) \rightarrow R F H_{a / 4}^{b}\left(\Sigma_{0}, V\right)
$$

and analogously

$$
\Phi_{a / 2}^{b} \circ \Psi_{a}^{b / 2}=\pi_{a / 4, a}^{b} \circ \iota_{a}^{b, b / 4}: R F H_{a}^{b / 4}\left(\Sigma_{1}, V\right) \rightarrow R F H_{a / 4}^{b}\left(\Sigma_{1}, V\right) .
$$


Hence we conclude that

$$
\begin{array}{ll}
\bar{\Psi} \circ \bar{\Phi}=\left.\mathrm{id}\right|_{\overline{R F H}\left(\Sigma_{0}, V\right)}, & \bar{\Phi} \circ \bar{\Psi}=\left.\mathrm{id}\right|_{\overline{R F H}\left(\Sigma_{1}, V\right)}, \\
\underline{\Psi} \circ \underline{\Phi}=\left.\mathrm{id}\right|_{R F H}\left(\Sigma_{0}, V\right), & \underline{\Phi} \circ \underline{\Psi}=\left.\mathrm{id}\right|_{R F H}\left(\Sigma_{1}, V\right) .
\end{array}
$$

This implies that $\bar{\Phi}$ and $\underline{\Phi}$ are isomorphisms with inverses

$$
\bar{\Phi}^{-1}=\bar{\Psi}, \quad \underline{\Phi}^{-1}=\underline{\Psi} .
$$

This finishes the proof of the theorem.

\section{Mañé's critical values}

In this section we summarize the main properties associated with the various Mañés critical values. We also show that there are open sets of hypersurfaces with high energy which are not stable; however these are virtually contact, showing the need to consider the latter notion.

\subsection{Definition and basic properties}

As in the Introduction we consider the cotangent bundle $\tau: T^{*} M \rightarrow M$ of a closed manifold $M$ and the autonomous Hamiltonian system defined by a convex Hamiltonian

$$
H(q, p)=\frac{1}{2}|p|^{2}+U(q)
$$

and a twisted symplectic form

$$
\omega_{\sigma}=\omega=d p \wedge d q+\tau^{*} \sigma .
$$

Here $d p \wedge d q$ is the canonical symplectic form in canonical coordinates $(q, p)$ on $T^{*} M,|p|$ denotes the dual norm of a Riemannian metric $g$ on $M, U: M \rightarrow \mathbb{R}$ is a smooth potential, and $\sigma$ is a closed 2-form on $M$. This Hamiltonian system describes the motion of a particle on $M$ subject to the conservative force $-\nabla U$ and the magnetic field $\sigma$. In local coordinates $q_{1}, \ldots, q_{n}$ on $M$ and dual coordinates $p_{1}, \ldots, p_{n}$ the Hamiltonian system is given by

$$
\begin{aligned}
& \dot{q}_{i}=\frac{\partial H}{\partial p_{i}}, \\
& \dot{p}_{i}=-\frac{\partial H}{\partial q_{i}}+\sum_{j=1}^{n} \sigma_{i j}(q) \frac{\partial H}{\partial p_{j}},
\end{aligned}
$$


where

$$
\sigma=\frac{1}{2} \sum_{i, j=1}^{n} \sigma_{i j}(q) d q_{i} \wedge d q_{j}, \quad \sigma_{i j}=-\sigma_{j i} .
$$

In particular, the $q$-components of the Hamiltonian vector field $X_{H}$ are independent of $\sigma$ :

$$
X_{H}=\left(H_{p}, *\right), \quad H_{p}=\frac{\partial H}{\partial p} .
$$

Let $\Pi: \widehat{M} \rightarrow M$ be a cover of $M$ and suppose that $\Pi^{*} \sigma$ is exact. The Hamiltonian $H$ lifts to Hamiltonian $\hat{H}$ and we define the Mañé critical value of the cover as

$$
c(\hat{H}):=\inf _{\theta} \sup _{q \in \widehat{M}} \hat{H}\left(q, \theta_{q}\right),
$$

where the infimum is taken over all 1 -forms $\theta$ on $\widehat{M}$ with $d \theta=\Pi^{*} \sigma$.

If $\bar{M}$ is a cover of $\widehat{M}$, then clearly $c(\bar{H}) \leq c(\hat{H})$ and equality holds if $\bar{M}$ is a finite cover of $\widehat{M}$.

The critical value may also be defined in Lagrangian terms. Consider the Lagrangian on $T \widehat{M}$ given by

$$
\widehat{L}(q, v)=\frac{1}{2}|v|^{2}-U(q)+\theta_{q}(v),
$$

where $\theta$ is any primitive of $\Pi^{*} \sigma$. It is well known that the extremals of $\hat{L}$, ie, the solutions of the Euler-Lagrange equations of $\hat{L}$,

$$
\frac{d}{d t} \frac{\partial \widehat{L}}{\partial v}(q, v)=\frac{\partial \widehat{L}}{\partial q}(q, v)
$$

coincide with the projection to $\widehat{M}$ of the orbits of the Hamiltonian $\widehat{H}$. The action of the Lagrangian $\widehat{L}$ on an absolutely continuous curve $\gamma:[a, b] \rightarrow \widehat{M}$ is defined by

$$
A_{\widehat{L}}(\gamma)=\int_{a}^{b} \hat{L}(\gamma(t), \dot{\gamma}(t)) d t .
$$

We define the Mañé critical value of the Lagrangian $\widehat{L}$ as (this was Mañé's original definition):

$c(\widehat{L})=\inf \left\{k \in \mathbb{R}: A_{\widehat{L}+k}(\gamma) \geq 0\right.$ for any absolutely continuous closed curve $\gamma$ defined on any closed interval $[0, T]\}$.

Note that $c(\widehat{L})$ may depend on the primitive $\theta$ chosen, but it will remain unchanged for all primitives of the form $\theta+d f$, thus it could only change if we consider another 
primitive $\theta^{\prime}$ such that $\theta-\theta^{\prime}$ determines a nonzero class in $H^{1}(\widehat{M}, \mathbb{R})$. The relationship between the Lagrangian and Hamiltonian critical values is given by (cf $[8 ; 20])$ :

$$
c(\hat{H})=\inf _{[\varpi] \in H^{1}(\widehat{M}, \mathbb{R})} c(\widehat{L}-\varpi) .
$$

There are two covers which are distinguished: the universal cover $\pi: \widetilde{M} \rightarrow M$ and the abelian cover $\pi_{0}: M_{0} \rightarrow M$. The latter is defined as the cover of $M$ whose fundamental group is the kernel of the Hurewicz homomorphism $\pi_{1}(M) \mapsto H_{1}(M, \mathbb{R})$ (we could also take coefficients in $\mathbb{Z}$; this will not alter the discussion below since critical values are unchanged by finite covers). We denote the lifts of $H$ to $\widetilde{M}$ resp. $M_{0}$ by $\widetilde{H}$ resp. $H_{0}$ and the corresponding Mañé critical values (when defined) by

$$
c:=c(\tilde{H})=c(\tilde{L}), \quad c_{0}:=c\left(H_{0}\right)=c\left(L_{0}\right) .
$$

The critical value $c=c(\tilde{H})=c(\tilde{L})$ is the one given in the Introduction. We note here some of its properties:

(i) $c<\infty$ if and only if $\pi^{*} \sigma$ has a bounded primitive (with respect to the lifted Riemannian metric).

(ii) If $M$ admits a metric of negative curvature, any closed 2-form $\sigma$ has bounded primitives in $\widetilde{M}$ [33].

(iii) If $[\sigma] \in H^{2}(M, \mathbb{R})$ is nonzero and $\pi_{1}(M)$ is amenable ${ }^{4}, c=\infty$ (see Gromov [33] and also Paternain [60, Corollary 5.4]).

Lemma 5.1 For any $k>c$, the hypersurface $\Sigma_{k}$ is virtually contact.

Proof If $k>c$ we may choose $\varepsilon>0$ and a primitive $\theta$ of $\pi^{*} \sigma$ such that

$$
\varepsilon+\left|\theta_{q}\right| \leq \sqrt{2(k-\tilde{U}(q))}
$$

for all $q \in \widetilde{M}$.

Let $\lambda$ be the Liouville form on $\widetilde{M}$. Then we may write $\widetilde{\omega}=d\left(\lambda+\widetilde{\tau}^{*} \theta\right)$. Since $X_{\tilde{H}}=\left(\widetilde{H}_{p}, *\right)$, on $\widetilde{\Sigma}_{k}$ we have

$$
\left(\lambda+\tilde{\tau}^{*} \theta\right)\left(X_{\tilde{H}}\right)=|p|^{2}+\theta_{q}\left(\tilde{H}_{p}\right)=2(k-\tilde{U}(q))+\theta_{q}\left(\tilde{H}_{p}\right) .
$$

\footnotetext{
${ }^{4}$ A group $G$ is amenable eg if it is abelian, solvable or nilpotent. If $G$ contains a free subgroup on two generators (which is the case eg for the fundamental group of a closed surface of genus at least 2) it is not amenable.
} 
But $\theta_{q}\left(\tilde{H}_{p}\right) \geq-\left|\theta_{q}\right| \sqrt{2(k-\tilde{U}(q))}$ for all $q \in \widetilde{M}$. It follows from (45) and (46) that

$$
\begin{aligned}
\left(\lambda+\tilde{\tau}^{*} \theta\right)\left(X_{\tilde{H}}\right) & \geq 2(k-\tilde{U}(q))-\left|\theta_{q}\right| \sqrt{2(k-\tilde{U}(q))} \\
& \geq \sqrt{2(k-\tilde{U}(q))}\left(\sqrt{2(k-\tilde{U}(q))}-\left|\theta_{q}\right|\right) \\
& \geq \varepsilon^{2} .
\end{aligned}
$$

On the other hand (45) also implies that there is a constant $C>0$ such that

$$
\sup _{(q, p) \in \widetilde{\Sigma_{k}}}\left|\left(\lambda+\widetilde{\tau}^{*} \theta\right)_{(q, p)}\right|<C
$$

and thus $\Sigma_{k}$ is virtually contact.

\subsection{Hyperbolic spaces}

The results in this subsection are well known; we include them here for the reader's convenience ( $\mathrm{cf}[29])$. Let $\Gamma$ be a cocompact lattice of $\operatorname{PSL}(2, \mathbb{R})$. The standard horocycle flow $h_{t}$ is given by the right action of the one-parameter subgroup

$$
\left(\begin{array}{ll}
1 & t \\
0 & 1
\end{array}\right)
$$

on $\Gamma \backslash \operatorname{PSL}(2, \mathbb{R})$. The horocycle flow is known to be uniquely ergodic [28]. Moreover, it has zero topological entropy since

$$
\phi_{t}^{0} \circ h_{s}=h_{s e^{-t}} \circ \phi_{t}^{0}
$$

for all $s, t \in \mathbb{R}$, where $\phi^{0}$ is the geodesic flow given by the one-parameter subgroup

$$
\left(\begin{array}{cc}
e^{t / 2} & 0 \\
0 & e^{-t / 2}
\end{array}\right)
$$

(The relation $\phi_{t}^{0} \circ h_{s}=h_{s e^{-t}} \circ \phi_{t}^{0}$ implies that for any $t$, the flows $s \mapsto h_{s}$ and $s \mapsto$ $h_{s e^{-t}}$ are conjugate and thus they must have the same entropy. But for $t \neq 0$, this forces the entropy of $h_{s}$ to be zero.)

In fact, $h_{t}$ parametrizes the strong stable manifolds of $\phi^{0}$. A matrix $X$ in $\mathfrak{s l}(2, \mathbb{R})$ gives rise to a flow $\phi$ on $\Gamma \backslash \operatorname{PSL}(2, \mathbb{R})$ by setting

$$
\phi_{t}(\Gamma g)=\Gamma g e^{t X} .
$$


The geodesic and horocycle flows are just particular cases of these algebraic flows. Consider the following path of matrices in $\mathfrak{s l}(2, \mathbb{R})$ :

$$
\mathbb{R} \ni s \mapsto X_{S}:=\left(\begin{array}{cc}
1 / 2 & 0 \\
0 & -1 / 2
\end{array}\right)+s\left(\begin{array}{cc}
0 & 1 / 2 \\
-1 / 2 & 0
\end{array}\right)
$$

The flows $\phi^{s}$ on $\Gamma \backslash \operatorname{PSL}(2, \mathbb{R})$ associated with the matrices $X_{s}$ can be interpreted as magnetic flows. Since $\operatorname{PSL}(2, \mathbb{R})$ acts by isometries on the hyperbolic plane $\mathbb{H}^{2}$, $M:=\Gamma \backslash \mathbb{H}^{2}$ is a compact hyperbolic surface provided $\Gamma$ acts without fixed points, and the unit sphere bundle $S M$ of $M$ can be identified with $\Gamma \backslash \operatorname{PSL}(2, \mathbb{R})$. A simple calculation shows that $\phi^{s}$ is the Hamiltonian flow of the Hamiltonian $H(x, v)=$ $(1 / 2)|v|_{x}^{2}$ with respect to the twisted symplectic form on $T M$ given by

$$
\omega_{s}=d \alpha-s \pi^{*} \sigma
$$

where $\sigma$ is the area form of $M, \pi: T M \rightarrow M$ is the canonical projection and $\alpha$ is the Liouville 1-form that generates the geodesic flow of $M$. For $s=0, \phi^{0}$ is the geodesic flow and for $s=1, \phi^{1}$ is the flow induced by the one-parameter subgroup with matrix on $\mathfrak{s l}(2, \mathbb{R})$ given by

$$
X_{1}=\left(\begin{array}{cc}
1 / 2 & 1 / 2 \\
-1 / 2 & -1 / 2
\end{array}\right)
$$

Observe that there exists an element $c \in \operatorname{PSL}(2, \mathbb{R})$ such that

$$
c^{-1} X_{1} c=\left(\begin{array}{ll}
0 & 1 \\
0 & 0
\end{array}\right) \text {. }
$$

Explicitly

$$
c=\frac{1}{\sqrt{2}}\left(\begin{array}{cc}
1 & 1 \\
-1 & 1
\end{array}\right) \text {. }
$$

Then the map $f: \Gamma \backslash \operatorname{PSL}(2, \mathbb{R}) \rightarrow \Gamma \backslash \operatorname{PSL}(2, \mathbb{R})$ given by $f(\Gamma g)=\Gamma g c$ conjugates $\phi^{1}$ and $h$, ie $f \circ \phi_{t}^{1}=h_{t} \circ f$. (In fact, any matrix in $\mathfrak{s l}(2, \mathbb{R})$ with determinant zero will give rise to a flow which is conjugate to $h_{t}$ or $h_{-t}$. So, up to orientation, there is just one algebraic horocycle flow.) Note that $\operatorname{det} X_{s}=-(1 / 4)\left(1-s^{2}\right)$, so for $|s|<1$, the flow $\phi^{s}$ is conjugate to the geodesic flow $\phi^{0}$, up to a constant time scaling by $\sqrt{1-s^{2}}$. Hence the magnetic flows $\phi^{s}$ are just geodesic flows, but with entropy $\sqrt{1-s^{2}}$. Similarly, for $|s|>1, \phi^{s}$ is up to a constant time scaling conjugate to the flow generated by

$$
V:=\left(\begin{array}{cc}
0 & 1 / 2 \\
-1 / 2 & 0
\end{array}\right)
$$

which is actually the fibrewise circle flow. 
In the discussion above we have kept the hypersurface $S M(k=1 / 2)$ fixed and changed $\sigma$ by $s \sigma$. This was just done for convenience, since the flow $\phi^{s}$ on $S M$ is equivalent to the Hamiltonian flow on $\Sigma_{k}$ with respect to $\sigma$, where $s^{2}=1 / 2 k$. Note that $\omega_{s}$ restricted to $S M$ has a primitive given by $\psi_{s}:=\alpha-s \gamma$, where $\gamma$ is the unique left-invariant 1-form which takes the value one on $V$ and zero on

$$
\left(\begin{array}{cc}
1 / 2 & 0 \\
0 & -1 / 2
\end{array}\right), \quad\left(\begin{array}{cc}
0 & -1 / 2 \\
-1 / 2 & 0
\end{array}\right) \text {. }
$$

The 1 -form $\gamma$ is also the connection 1-form determined by the Levi-Civita connection of the hyperbolic metric. Also note that $\psi_{s}\left(X_{S}\right)=1-s^{2}$ and thus $\Sigma_{k}$ is of contact type for all $k \neq 1 / 2$ (with opposite orientation for $k<1 / 2$ ).

The fact that the Mañé critical value is $c=1 / 2$ can be seen as follows. The form $y^{-1} d x$ is a primitive of the area form $y^{-2} d x \wedge d y$ and has norm 1 . This shows that $c \leq 1 / 2$. To see that in fact $c=1 / 2$ one performs a calculation with geodesic circles exactly as the one in the proof of Lemma 6.11 below.

Summarizing, we have obtained the picture described in the Introduction (Section 1):

- For $k>1 / 2$, the dynamics is Anosov and conjugate (after rescaling) to the underlying geodesic flow. The energy levels are contact.

- At the Mañé critical value $k=1 / 2$ we hit the horocycle flow. There are no closed orbits and the level is unstable.

- For $k<1 / 2$ all orbits are closed and contractible. Energy levels are contact but with opposite orientation.

A very similar picture is obtained if we take compact quotients of complex hyperbolic space $\mathbb{H}_{\mathbf{C}}^{n}$ with its Kähler form; see Dairbekov and Paternain [22, Appendix]. If we normalize the Kähler structure $(g, \sigma)$ to have holomorphic sectional curvature -1 (so the sectional curvature $K$ satisfies $-1 \leq K \leq-1 / 4$ ), then the Mañé critical value is $c=1 / 2, \Sigma_{k}$ is Anosov for $k>1 / 2$, for $k=1 / 2$ one obtains a unipotent ergodic flow without closed orbits, and for $k<1 / 2$ all orbits are closed and contractible. However, there is an essential difference between the case $n=1$ and $n \geq 2$. As we saw above, for $n=1$ the restriction of the symplectic form to $\Sigma_{k}$ is exact. This is no longer the case for $n \geq 2$. Nevertheless it is easy to see that for $k>1 / 2, \Sigma_{k}$ is stable. Indeed, since the flow is algebraic, the strong stable and unstable bundles are real analytic and together they span a hyperplane bundle $\xi$ in $\Sigma_{k}$ invariant under the magnetic flow. If we define a 1 -form $\lambda$ such that $\lambda\left(X_{H}\right)=1$ and $\xi=\operatorname{ker} \lambda$, then $\lambda$ is a stabilizing 1 -form. Observe that in this case $d \lambda$ and $\omega$ span a 2-dimensional space of flow invariant 2 -forms. For $n=1$ this space is just one dimensional. 


\subsection{The exact case}

Now we focus on the case that $\sigma$ is exact. We begin with the case $\sigma=0$.

Lemma 5.2 Suppose $\sigma=0$. Then $c=\max U$ and any regular level set $\Sigma_{k}$ is of contact type.

Proof If $\sigma=0$ the infimum in the definition of $c=c(\hat{H})$ is attained for $\theta=0$, hence $c=\max U$ and $k=c$ is a singular value of $H$. If $k>c=\max U$, then $\Sigma_{k}$ encloses the Lagrangian zero section and is thus of contact type (with the Liouville form $\lambda$ as contact form). Suppose then $k<c$ is a regular value. A quick glance at the proof of the previous lemma shows that $\lambda\left(X_{H}\right)=|p|^{2} \geq 0$. Note that the set $\{p=0\} \cap \Sigma_{k}$ does not contain any set invariant under the flow of $X_{H}$ since $X_{H}(q, 0)=(0, *) \neq 0$. Therefore, for any invariant Borel probability measure $\mu$ we have

$$
\int_{\Sigma_{k}} \lambda\left(X_{H}\right) d \mu>0
$$

and $\Sigma_{k}$ is of contact type by Theorem 2.7. Alternatively, the condition $X_{H}(q, 0)=$ $(0, *) \neq 0$ allows us to pick a function $f: T^{*} M \rightarrow \mathbb{R}$ with $d f\left(X_{H}\right)>0$ along $\{p=0\} \cap \Sigma_{k}$, hence (for $f$ small) $(\lambda+d f)\left(X_{H}\right)>0$ and $\lambda+d f$ is a contact form on $\Sigma_{k}$.

More generally, we have:

Lemma 5.3 A closed 2-form $\sigma$ on $M$ is exact if and only if $\pi_{0}^{*} \sigma$ has a bounded primitive. The Mañé critical value $c_{0}$ associated to the abelian cover $\pi_{0}: M_{0} \rightarrow M$ is given by the formula in the Introduction (Section 1)

$$
c_{0}=c(H)=\inf _{\theta} \sup _{q \in M} H\left(q, \theta_{q}\right),
$$

where the infimum is taken over all 1 -forms $\theta$ on $M$ with $d \theta=\sigma$ (and $c_{0}=\infty$ if $\sigma$ is not exact). If $k>c_{0}$ the hypersurface $\Sigma_{k}$ is of contact type.

Proof The first two statements follow from the fact that the deck transformation group of the cover $\pi_{0}: M_{0} \rightarrow M$ is abelian, hence amenable, so a bounded primitive of $\pi_{0}^{*} \sigma$ can be averaged to a primitive of $\sigma$ on $M$ [33;60, Corollary 5.4]. The proof of the last statement is analogous to the proof of Lemma 5.1 (but simpler).

Remark Note that in the last two lemmas we actually obtain restricted contact type. 
Relation to Mather's $\alpha$-function Suppose now that $\sigma$ is exact and fix a primitive $\theta$ in $M$ and consequently a Lagrangian $L$. As Mañé pointed out [49; 19], there is a close relationship between the critical values and Mather's minimizing measures [50]. Mather's $\alpha$ function is a convex superlinear function $\alpha: H^{1}(M, \mathbb{R}) \rightarrow \mathbb{R}$ given by

$$
\alpha([\varpi])=-\min _{\mu} \int_{T M}(L-\varpi) d \mu
$$

where $\mu$ runs over all Borel probability measures on $T M$ invariant under the EulerLagrange flow of $L$. It turns out that

$$
\alpha([\varpi])=c(L-\varpi)
$$

and therefore $[61 ; 20]$

$$
c_{0}=c(H)=\min _{[\varpi] \in H^{1}(M, \mathbb{R})} \alpha([\varpi]) .
$$

The value $c_{0}=c(H)$ is also called the strict critical value. We now summarize some of the main properties of $c_{0}$ :

(i) The value $c_{0}=c\left(L_{0}\right)=c\left(H_{0}\right)$, where $L_{0}, H_{0}$ are the lifts of $L, H$ to the abelian cover $M_{0}$ [61].

(ii) If $M \neq \mathbb{T}^{2}$ and $c<k \leq c_{0}$, then $\Sigma_{k}$ is not of contact type [18, Theorem B.1].

(iii) There is a characterization of $c_{0}$ in terms of Symplectic Topology [62]: it is the infimum of the values of $k$ such that the region bounded by $\Sigma_{k}$ contains a Lagrangian submanifold Hamiltonian isotopic to $\operatorname{gr}(-\theta)$. (in fact the whole of Mather's $\alpha$ function can be characterized in a similar way just by considering Lagrangian submanifolds with a fixed Liouville class).

(iv) If $\pi_{1}(M)$ is amenable, $c=c_{0}$ [24].

The gap between $c$ and $c_{0}$ The inequality $c \leq c_{0}$ could be strict. Examples of this phenomenon were given for the first time in [61]:

Theorem 5.4 [61] On a closed oriented surface $M$ of genus $\geq 2$ there exists a metric of negative curvature and an exact 2-form $\sigma$ such that $c<c_{0}$. Moreover, there exists an open interval $I \subset\left(c, c_{0}\right)$ such that all level sets $\Sigma_{k}$ with $k \in I$ are Anosov. In particular, these levels are virtually contact but not stable.

Proof All statements except the last one are proven in [61]. The levels $k \in I$ are virtually contact by Lemma 5.1. On the other hand, suppose a level $k \in I$ is stable and let $\lambda$ be a stabilizing 1 -form. The transitivity of the flow and the fact that $\operatorname{dim} M=2$ easily imply the existence of a constant $a$ such that $d \lambda=a \omega$. If $a \neq 0$ we obtain a 
contradiction with the fact that these energy levels cannot be contact by property (ii) above. If $a=0$, we obtain a contradiction with Corollary 2.10 .

Next we describe a large class of manifolds exhibiting a large gap between $c$ and $c_{0}$. Consider a closed manifold $M$ with a closed 2 -form $\Omega$ such that $0 \neq[\Omega] \in H^{2}(M, \mathbb{Z})$. Suppose moreover that $\Omega$ has a bounded primitive in $\widetilde{M}$. Consider a circle bundle $p: P \rightarrow M$ with Euler class $[\Omega]$ and $\psi$ a connection 1 -form with $p^{*} \Omega=-d \psi$. Fix any Riemannian metric $h$ on $M$ and consider for $\varepsilon>0$ the metric $g_{\varepsilon}$ on $P$ given by

$$
g_{\varepsilon}(u, v)=\varepsilon^{-1} h(d p(u), d p(v))+\psi(u) \psi(v) .
$$

Theorem 5.5 For $H=(1 / 2)|p|_{\varepsilon}^{2}$ on $T^{*} P$ and $\sigma=-d \psi$ the strict Mañé critical value satisfies $c_{0}\left(g_{\varepsilon}, \sigma\right)=1 / 2$ for all $\varepsilon>0$, but $c\left(g_{\varepsilon}, \sigma\right) \rightarrow 0$ as $\varepsilon \rightarrow 0$.

Proof Since $[\Omega] \neq 0$, the Gysin sequence of the circle bundle $p: P \rightarrow M$ shows that $p^{*}: H^{1}(M, \mathbb{R}) \rightarrow H^{1}(P, \mathbb{R})$ is an isomorphism. Thus the orbits of the circle action are all null-homologous in $H_{1}(P ; \mathbb{R})$. Let $V$ be the vector field dual to $\psi$ and note that $g_{\varepsilon}(V, V)=1$. Clearly this implies $c_{0}\left(g_{\varepsilon}, \sigma\right) \leq 1 / 2$. To show that equality holds we consider the Lagrangian on $P$ given by

$$
L(q, v)=\frac{1}{2}|v|_{\varepsilon}^{2}-\psi_{q}(v),
$$

and we note that the action $A_{L+k}$ of the orbits of $V$ equals $2 \pi(k-1 / 2)$. Since the orbits of $V$ are null-homologous in $H_{1}(P ; \mathbb{R})$ and this action is negative if $k<1 / 2$ we must have $c_{0}\left(g_{\varepsilon}, \sigma\right)=1 / 2$.

Let $\pi: \widetilde{M} \rightarrow M$ be the universal cover and $\hat{p}: \pi^{*} P \rightarrow \widetilde{M}$, the pullback bundle. Let $\hat{\pi}: \pi^{*} P \rightarrow P$ be the obvious map such that $p \circ \hat{\pi}=\pi \circ \hat{p}$. Clearly $\hat{\pi}$ is a covering map of $P$. By hypothesis, we may write $\pi^{*} \Omega=d \theta$, where $|\theta|_{\infty}=\sup _{q \in \widetilde{M}}\left|\theta_{q}\right|<\infty$. Note that

$$
\begin{gathered}
d \hat{p}^{*} \theta=-d \hat{\pi}^{*} \psi=\hat{\pi}^{*} \sigma \\
\left|\theta_{q}\right|_{\varepsilon}=\min _{|v|_{\varepsilon}=1}\left|\theta_{q}(v)\right|=\varepsilon \min _{|v|=1}\left|\theta_{q}(v)\right|=\varepsilon\left|\theta_{q}\right| .
\end{gathered}
$$

and that

Thus

$$
c\left(g_{\varepsilon}, \sigma\right) \leq \frac{\varepsilon|\theta|_{\infty}^{2}}{2} \longrightarrow 0 \text { as } \varepsilon \rightarrow 0 .
$$

This proves the Theorem.

Later on in Section 6 we shall see another very explicit example which together with the preceding result suggests that the gap between $c$ and $c_{0}$ is rather frequent on manifolds with nonamenable fundamental groups. 


\subsection{Instability for large energies when $[\sigma] \neq 0$}

The purpose of this subsection is to show that when $[\sigma] \neq 0$, there can be hypersurfaces $\Sigma_{k}$ which are not stable for any sufficiently large $k$. More precisely, we will prove:

Theorem 5.6 Let $(M, g)$ be a closed Riemannian manifold of even dimension different from two whose sectional curvature satisfies the pinching condition $-4 \leq K<-1$. Let $\sigma$ be a closed $2-$ form with $[\sigma] \neq 0$. Then for any $k$ sufficiently large, the hypersurface $\Sigma_{k}$ is not stable.

Remark Note that these levels are actually virtually contact by Lemma 5.1 and the fact that $\sigma$ has bounded primitives on the universal cover of $M$.

The ideas for the proof of this theorem come from $[26 ; 25 ; 34 ; 41]$.

We start with a preliminary discussion on Anosov Hamiltonian structures.

Recall that the flow $\phi_{t}$ of a vector field $F$ on a manifold $\Sigma$ is Anosov if there is a splitting $T \Sigma=\mathbb{R} F \oplus E^{s} \oplus E^{u}$ and positive constants $\lambda$ and $C$ such that for all $x \in \Sigma$,

$$
\begin{aligned}
\left|d_{x} \phi_{t}(v)\right| \leq C e^{-\lambda t}|v| & \text { for } v \in E^{s} \text { and } t \geq 0, \\
\left|d_{x} \phi_{-t}(v)\right| \leq C e^{-\lambda t}|v| & \text { for } v \in E^{u} \text { and } t \geq 0 .
\end{aligned}
$$

If an Anosov vector field $F$ is rescaled by a positive function its flow remains Anosov [3; 59]. It will be useful for us to know how the bundles $E^{s}$ and $E^{u}$ change when we rescale $F$ by a smooth positive function $r: \Sigma \rightarrow \mathbb{R}_{+}$. Let $\tilde{\phi}$ be the flow of $r F$ and $\widetilde{E}^{s}$ its stable bundle. Then (cf [59])

$$
\widetilde{E}^{s}(x)=\left\{v+z(x, v) F(x): v \in E^{s}(x)\right\},
$$

where $z(x, v)$ is a continuous 1 -form (ie linear in $v$ and continuous in $x)$. Moreover, if we let $l=l(t, x)$ be (for fixed $x$ ) the inverse of the diffeomorphism

$$
t \mapsto \int_{0}^{t} r\left(\phi_{s}(x)\right)^{-1} d s
$$

then

$$
d \tilde{\phi}_{t}(v+z(x, v) F(x))=d \phi_{l}(v)+z\left(\phi_{l}(v), d \phi_{l}(v)\right) F\left(\phi_{l}(x)\right) .
$$

There is a similar expression for $\widetilde{E}^{u}$. It is clear from the discussion above that the weak bundles $\mathbb{R} F \oplus E^{s}$ and $\mathbb{R} F \oplus E^{u}$ do not change under rescaling of $F$ (the strong bundles $E^{s, u}$ are indeed affected by rescaling as we have just seen). 
Let $(\Sigma, \omega)$ be a Hamiltonian structure. We say that the structure is Anosov if the flow of any vector field $F$ spanning $\operatorname{ker} \omega$ is Anosov.

We say that an Anosov Hamiltonian structure satisfies the 1/2-pinching condition or that it is $1-$ bunched $[36 ; 35]$ if for any vector field $F$ spanning $\operatorname{ker} \omega$ with flow $\phi_{t}$ there are functions $\mu_{f}, \mu_{s}: \Sigma \times \mathbb{R}_{+} \rightarrow \mathbb{R}_{+}$such that

- $\lim _{t \rightarrow \infty} \sup _{x \in \Sigma} \mu_{s}(x, t)^{2} / \mu_{f}(x, t)=0$;

- $\mu_{f}(x, t)|v| \leq\left|d \phi_{t}(v)\right| \leq \mu_{s}(x, t)|v|$ for all $x \in \Sigma, t>0$ and $v \in E^{s}(x)$, and $\mu_{f}(x, t)|v| \leq\left|d \phi_{-t}(v)\right| \leq \mu_{s}(x, t)|v|$ for all $x \in \Sigma, t>0$ and $v \in E^{u}\left(\phi_{t} x\right)$.

We remark that the $1 / 2-$ pinching condition is invariant under rescaling. Indeed, consider the flow $\widetilde{\phi}_{t}$ of $r F$. It is clear from (47) and (48) that there is a positive constant $\kappa$ such that

$$
\frac{1}{\kappa} \mu_{f}(x, l(t, x))|\widetilde{v}| \leq\left|d \widetilde{\phi}_{t}(\widetilde{v})\right| \leq \kappa \mu_{s}(x, l(t, x))|\widetilde{v}|
$$

for $t>0$ and $\tilde{v} \in \widetilde{E}^{s}$ (with a similar expression for $\widetilde{E}^{u}$ ). We know that given $\varepsilon>0$, there exists $T>0$ such that for all $x \in \Sigma$ and all $t>T$ we have

$$
\frac{\mu_{s}(x, t)^{2}}{\mu_{f}(x, t)}<\varepsilon .
$$

On the other hand, there exists $a>0$ such that $l(t, x) \geq a t$ for all $x \in \Sigma$ and $t>0$. Hence for all $t>T / a$ we have

$$
\frac{\mu_{s}(x, l(t, x))^{2}}{\mu_{f}(x, l(t, x))}<\varepsilon
$$

for all $x \in \Sigma$. Therefore

$$
\lim _{t \rightarrow \infty} \sup _{x \in \Sigma} \frac{\mu_{s}(x, l(t, x))^{2}}{\mu_{f}(x, l(t, x))}=0
$$

and thus $\widetilde{\phi}_{t}$ is also $1 / 2-$ pinched.

Hence the Anosov property as well as the 1/2-pinching condition are invariant under rescaling and thus intrinsic properties of the Hamiltonian structure. One of the main consequences of the 1/2-pinching condition is that the weak bundles $\mathbb{R} F \oplus E^{s}$ and $\mathbb{R} F \oplus E^{u}$ are of class $C^{1}$ by Hasselblatt [35, Theorem 5] (see also Hirsch, Pugh and Shub [37]).

Suppose now $(\Sigma, \omega)$ is a stable Anosov Hamiltonian structure satisfying the $1 / 2-$ pinching condition and let $\lambda$ be the stabilizing $1-$ form and $R$ the Reeb vector field. 
Invariance under the flow implies that $\omega$ and $\lambda$ both vanish on $E^{s}$ and $E^{u}$. Since the flow $\phi_{t}$ of $R$ is Anosov and $E^{s} \oplus E^{u}=\operatorname{ker} \lambda$ which is $C^{\infty}$, it is clear that $E^{s}$ and $E^{u}$ must be $C^{1}$ since $E^{s, u}=\left(\mathbb{R} F \oplus E^{s, u}\right) \cap \operatorname{ker} \lambda$. Under these conditions we can introduce the Kanai connection [41] which is defined as follows.

Let $I$ be the $(1,1)$-tensor on $\Sigma$ given by $I(v)=-v$ for $v \in E^{s}, I(v)=v$ for $v \in E^{u}$ and $I(R)=0$. Consider the symmetric nondegenerate bilinear form given by

$$
h(X, Y):=\omega(X, I Y)+\lambda \otimes \lambda(X, Y) .
$$

The pseudo-Riemannian metric $h$ is of class $C^{1}$ and thus there exists a unique $C^{0}$ affine connection $\nabla$ such that

(i) $h$ is parallel with respect to $\nabla$;

(ii) $\nabla$ has torsion $\omega \otimes R$.

This connection has the following desirable properties $[25 ; 41]$ :

- The connection is invariant under $\phi_{t}$.

- The Anosov splitting is invariant under $\nabla$ : if $X$ is any section of $E^{s, u}, \nabla_{v} X \in$ $E^{s, u}$ for any $v$.

- The restriction of $\nabla$ to the weak stable and unstable manifolds (ie leaves of the weak stable and unstable foliations) is flat.

- Parallel transport along curves on weak stable/unstable manifolds coincide with the holonomy transport determined by the stable/unstable foliations.

The other good consequence of the 1/2-pinching condition, besides $C^{1}$ smoothness of the bundles, is the following lemma (cf [41, Lemma 3.2]).

Lemma $5.7 \nabla(d \lambda)=0$.

Proof Suppose $\tau$ is any invariant $(0,3)$-tensor annihilated by $R$. We claim that $\tau$ must vanish. To see this, consider for example a triple of vectors $\left(v_{1}, v_{2}, v_{3}\right)$ where $v_{1}, v_{2} \in E^{s}$ but $v_{3} \in E^{u}$. Then there is a constant $C>0$ such that

$$
\begin{aligned}
\left|\tau_{x}\left(v_{1}, v_{2}, v_{3}\right)\right| & =\left|\tau_{\phi_{t} x}\left(d \phi_{t}\left(v_{1}\right), d \phi_{t}\left(v_{2}\right), d \phi_{t}\left(v_{3}\right)\right)\right| \\
& \leq C \mu_{s}(x, t)^{2} \mu_{f}(x, t)^{-1}\left|v_{1}\right|\left|v_{2}\right|\left|v_{3}\right|
\end{aligned}
$$

By the 1/2-pinching condition the last expression tends to zero as $t \rightarrow \infty$ and therefore $\tau_{x}\left(v_{1}, v_{2}, v_{3}\right)=0$. The same will happen for other possible triples $\left(v_{1}, v_{2}, v_{3}\right)$ when we let $t \rightarrow \pm \infty$. 
Since $d \lambda$ and $\nabla$ are $\phi_{t}$-invariant, so is $\nabla(d \lambda)$. Since $i_{R} d \lambda=0, \nabla(d \lambda)$ is also annihilated by $R$ (to see that $\nabla_{R}(d \lambda)=0$ use that $d \lambda$ is $\phi_{t}$-invariant and that $\left.\nabla_{R}=L_{R}\right)$. Hence by the previous argument applied to $\tau=\nabla(d \lambda)$ we conclude that $\nabla(d \lambda)=0$ as desired.

Since $\omega$ is nondegenerate, there exists a smooth bundle map $L: E^{s} \oplus E^{u} \rightarrow E^{s} \oplus E^{u}$ such that for sections $X, Y$ of $E^{s} \oplus E^{u}$

$$
d \lambda(X, Y)=\omega(L X, Y)=\omega(X, L Y) .
$$

The map $L$ is invariant under $\phi_{t}$ and preserves the decomposition $E^{s} \oplus E^{u}$, ie $L=L^{s}+L^{u}$, where $L^{s}: E^{s} \rightarrow E^{s}$ and $L^{u}: E^{u} \rightarrow E^{u}$. In particular, $L$ commutes with $I$.

Suppose now $\operatorname{dim} \Sigma=2 n-1$, where $n$ is an even integer. Since $\operatorname{dim} E^{s}=n-1$ is odd, $L_{x}^{S}$ admits a real eigenvalue $\rho$ (note that by transitivity of $\phi_{t}$, the characteristic polynomial of $L_{x}^{s}$ is independent of $\left.x \in \Sigma\right)$. Let

$$
H(x):=\left\{v \in E^{s}(x): L_{x}^{s} v=\rho v\right\} .
$$

Since $\nabla(d \lambda)=0$ (Lemma 5.7), $H(x)$ is invariant under the parallel transport of $\nabla$ and thus $x \mapsto H(x)$ is a $C^{1}$ subbundle of $E^{s}$.

Let $W^{s}(x)$ be the strong stable manifold through $x$. We note that the restriction of $H$ to $W^{s}(x)$ is integrable. Indeed, let $X$ and $Y$ be parallel sections of $E^{s}$ over $W^{s}(x)$ (such sections must be $C^{1}$ ) and observe that since $\nabla$ has zero torsion on $E^{s}$ we have $[X, Y]=\nabla_{X} Y-\nabla_{Y} X=0$.

The maximal integral submanifolds of $H$ on $W^{s}(x)$ define a foliation of class $C^{1}$ on $W^{s}(x)$ and thus a foliation $\mathcal{F}$ of class $C^{1}$ on $\Sigma$.

Proof of Theorem 5.6 Suppose the sectional curvature $K$ of a Riemannian metric satisfies $-A^{2} \leq K \leq-a^{2}$ for some positive constants $a$ and $A$. Then, comparison theorems show that, by Klingenberg [43, Theorem 3.2.17] (see also Knieper [44, Proposition 3.2]) there is a constant $C>0$ such that

$$
\begin{array}{ll}
\frac{1}{C}|v| e^{-A t} \leq\left|d_{x} \phi_{t}(v)\right| \leq C|v| e^{-a t} & \text { for } v \in E^{s} \text { and } t \geq 0, \\
\frac{1}{C}|v| e^{-A t} \leq\left|d_{x} \phi_{-t}(v)\right| \leq C|v| e^{-a t} & \text { for } v \in E^{u} \text { and } t \geq 0,
\end{array}
$$

where $\phi_{t}$ is the geodesic flow of the Riemannian metric. If we let $\mu_{s}=C e^{-a t}$ and $\mu_{f}=(1 / C) e^{-A t}$ we see that $\phi_{t}$ is $1 / 2-$ pinched as long as $A<2 a$. Therefore the 
geodesic flow of a metric whose sectional curvature satisfies $-4 \leq K<-1$ is $1 / 2-$ pinched. For sufficiently large $k,\left(\Sigma_{k}, \omega_{\sigma}\right)$ is an Anosov Hamiltonian structure since it can be seen as a perturbation of the geodesic flow. In fact it is also $1 / 2$-pinched. This can be seen as follows. An equivalent claim is that $\left(\Sigma_{1 / 2}, \omega_{s \sigma}\right)$ is $1 / 2-$ pinched for small $s$. An inspection of the proof of (49) and (50) in [43, Theorem 3.2.17] shows that if we do the same analysis for the magnetic Jacobi (or Riccati) equation we obtain numbers $A(s)$ and $a(s)$ for which (49) and (50) hold. These numbers will be as close as we wish to $A(0)=2$ and $a(0)=\sqrt{-\max K}>1$ if $s$ is small enough and the $1 / 2$-pinching condition follows.

We now make some remarks concerning the space of leaves of the weak foliations. The weak unstable foliation $\mathcal{W}^{u}$ of $\left(\Sigma_{k}, \omega_{\sigma}\right)$ is transverse to the fibres of the fibration by $(n-1)$-spheres given by

$$
\tau: \Sigma_{k} \rightarrow M \text {. }
$$

(This simply follows from the fact that this property is true for the geodesic flow.) Let $\widetilde{M}$ denote the universal cover of $M$ and let $\widetilde{\Sigma}_{k}$ denote the preimage of $\Sigma_{k}$ in $T^{*} \widetilde{M}$. We also have a fibration by $(n-1)-$ spheres

$$
\tilde{\tau} \mid \tilde{\Sigma}_{k}: \widetilde{\Sigma}_{k} \rightarrow \widetilde{M}
$$

Let $\widetilde{\mathcal{W}}^{u}$ be the lifted foliation and note that the foliation $\widetilde{\mathcal{W}}^{u}$ is also transverse to the fibration $\tilde{\tau} \mid \widetilde{\Sigma}_{k}: \widetilde{\Sigma}_{k} \rightarrow \widetilde{M}$. Since the fibres are compact a standard result in foliations [11, page 91] implies that for every $p \in \widetilde{\Sigma}_{k}$ the map

$$
\left.\widetilde{\tau}\right|_{\widetilde{\mathcal{W}}^{u}(p)}: \widetilde{\mathcal{W}}^{u}(p) \rightarrow \widetilde{M}
$$

is a covering map. Since $\widetilde{M}$ is simply connected, $\widetilde{\tau} \mid \widetilde{\mathcal{W}}^{u}(p)$ is in fact a diffeomorphism and $\widetilde{\mathcal{W}}^{u}(p)$ is simply connected. Consequently, $\widetilde{\mathcal{W}}^{u}(p)$ intersects each fibre of the fibration $\widetilde{\tau} \mid \widetilde{\Sigma}_{k}: \widetilde{\Sigma}_{k} \rightarrow \widetilde{M}$ at just one point and therefore the space of leaves $B^{u}:=$ $\tilde{\Sigma}_{k} / \widetilde{\mathcal{W}}^{u}$ of the weak unstable foliation can be identified topologically with the $(n-1)-$ sphere. Similarly the space of leaves of the weak stable foliation is also an $(n-1)-$ sphere. Note that $\pi_{1}(M)$ acts on $B^{u}$. Since the characteristic foliation of $\left(\Sigma_{k}, \omega_{\sigma}\right)$ is topologically conjugate to that of the geodesic flow, the action of $\pi_{1}(M)$ on the space of leaves is topologically like in the geodesic flow case: Every element in $\pi_{1}(M)$ acts on $B^{u}$ as a north-south dynamics, ie a homeomorphism of $S^{n-1}$ with exactly two fixed points $P_{ \pm}$such that every other point converges to $P_{ \pm}$under forward resp. backward iteration.

Now suppose that $\Sigma_{k}$ is stable. Our previous discussion produces a foliation $\mathcal{F}$ of class $C^{1}$ on $\Sigma_{k}$. This foliation can be lifted to $\widetilde{\Sigma}_{k}$ and then projected to $B^{u}$ ( $\mathcal{F}$ is invariant under holonomy maps) to produce a $C^{1}$ foliation of positive dimension on $B^{u}$ which 
is $\pi_{1}(M)$-invariant. By the result in [27], such a foliation must be trivial, ie consist of just one leaf. This implies that $H=E^{s}$, and hence

$$
d \lambda=\rho \omega_{\sigma}
$$

for some constant $\rho$. By Corollary 2.10, the constant $\rho$ cannot be zero. It follows then that $\omega_{\sigma}$ must be exact on $\Sigma_{k}$. However, the Gysin sequence of the sphere bundle shows that this is impossible since $[\sigma] \neq 0$ and $n \neq 2$.

Thus $\Sigma_{k}$ cannot be stable for high energies, which proves Theorem 5.6.

Remark The proof above can be considerably improved following the ideas in [34] to show the following result: suppose $[\sigma] \neq 0$ and $n \geq 3$ (not necessarily even). If $\left(\Sigma_{k}, \omega_{\sigma}\right)$ is a 1/2-pinched Anosov Hamiltonian structure, then it cannot be stable [56]. If we drop the $1 / 2$-pinching condition, the result is no longer true since compact quotients of complex hyperbolic space with the Kähler form are stable for high energies (cf Section 5.2) It is tempting to conjecture that these are the only stable Anosov Hamiltonian structures with $[\sigma] \neq 0$ and $n \geq 3$.

\section{Homogeneous examples}

In this section we study magnetic flows for left-invariant metrics on some compact homogeneous spaces $\Gamma \backslash G$ and verify the paradigms in the introduction. Here $G$ is always a Lie group and $\Gamma \subset G$ a cocompact lattice, ie a discrete subgroup such that the left quotient $\Gamma \backslash G$ is compact. The magnetic field $\sigma$ will be always a left-invariant 2-form.

Using left translations we identify $T^{*} G$ with $G \times \mathfrak{g}^{*}$ so left-invariant smooth functions $f: T^{*} G \rightarrow \mathbb{R}$ are just elements in $C^{\infty}\left(\mathfrak{g}^{*}\right)$. As before we set $\omega_{\sigma}=d p \wedge d q+\tau^{*} \sigma$, where $d p \wedge d q$ is the canonical symplectic form in $T^{*} G$. Let $\{,\} \sigma$ be the Poisson bracket of $\omega_{\sigma}$ defined as $\{H, F\}_{\sigma}=\omega_{\sigma}\left(X_{H}, X_{F}\right)$. Since all the objects involved are left-invariant, in the next lemma we just focus on the identity $e \in G$.

Lemma 6.1 Let $\mu \in \mathfrak{g}^{*},(v, \xi),(w, \eta) \in T_{(e, \mu)}\left(G \times \mathfrak{g}^{*}\right)=\mathfrak{g} \times \mathfrak{g}^{*}$. We have that

(i) $\omega_{\sigma}(e, \mu)((v, \xi),(w, \eta))=\xi(w)-\eta(v)-\mu([v, w])+\sigma_{e}(v, w)$;

(ii) given $f, g \in C^{\infty}\left(\mathfrak{g}^{*}\right)$,

$$
\{f, g\}_{\sigma}(\mu)=\mu\left(\left[d_{\mu} f, d_{\mu} g\right]\right)-\sigma_{e}\left(d_{\mu} f, d_{\mu} g\right)
$$

where we canonically identify $\left(\mathfrak{g}^{*}\right)^{*}$ with $\mathfrak{g}$; 
(iii) given $f \in C^{\infty}\left(\mathfrak{g}^{*}\right)$, if $X_{f}$ be the Hamiltonian vector field of $f$ with respect to $\omega_{\sigma}$, then

$$
X_{f}(e, \mu)=\left(d_{\mu} f, E_{f}(\mu)\right),
$$

where $E_{f}(\mu) \in \mathfrak{g}^{*}$ is given by

$$
E_{f}(\mu)(w)=\mu\left(\left[d_{\mu} f, w\right]\right)-\sigma_{e}\left(d_{\mu} f, w\right) .
$$

We will call $E_{f}$ the Euler vector field of $f$.

Proof The proof of the lemma for $\sigma=0$ can be found in [2, Section 4.4]. When $\sigma \neq 0$ the lemma follows right away from the definition of $\omega_{\sigma}$; we leave the details to the reader.

Suppose $f \in C^{\infty}\left(\mathfrak{g}^{*}\right)$ has a compact level set $\mathbf{S}_{k}:=f^{-1}(k) \subset \mathfrak{g}^{*}$. Clearly $E_{f}$ is tangent to $\mathbf{S}_{k}$; let $\psi_{t}$ be its flow. Let $\phi_{t}$ be the flow of $X_{f}$ in $\Sigma_{k}=(\Gamma \backslash G) \times \mathbf{S}_{k}$. Let $\pi_{2}:(\Gamma \backslash G) \times \mathbf{S}_{k} \rightarrow \mathbf{S}_{k}$ be the second factor projection. Clearly $\pi_{2} \circ \phi_{t}=\psi_{t} \circ \pi_{2}$. Thus, if $E_{f}$ is geodesible on $\mathbf{S}_{k}$, then $\Sigma_{k}$ is a stable hypersurface. This observation is nothing but a rephrasing of Lemma 2.3 in this context.

\subsection{Tori}

Consider the torus $\mathbb{T}^{n}(n \geq 2)$ and let $\sigma$ be a nonzero constant 2 -form. Let $\omega_{\sigma}=$ $d p \wedge d q+\tau^{*} \sigma$ be the twisted symplectic form on $T^{*} \mathbb{T}^{n}$. We shall consider on $\mathbb{T}^{n}$ the usual flat metric and we let $\Sigma_{k}:=H^{-1}(k)$, where $H(q, p)=(1 / 2)|p|^{2}$. Below we will make use of the following elementary lemma.

Lemma 6.2 Consider $\mathbb{R}^{k}$ with its usual inner product and let $A: \mathbb{R}^{k} \rightarrow \mathbb{R}^{k}$ be an antisymmetric linear map. Consider the 1 -form $\alpha$ in $\mathbb{R}^{k}$ given by $\alpha_{p}(\xi)=(1 / 2)\langle p, A \xi\rangle$. Then

$$
d \alpha_{p}\left(\xi_{1}, \xi_{2}\right)=\left\langle\xi_{1}, A \xi_{2}\right\rangle
$$

Proposition 6.3 If $\sigma$ is nonzero, then the hypersurface $\Sigma_{k}$ is stable, tame and displaceable for any $k>0$.

Proof Write $T^{*} \mathbb{T}^{n}=\mathbb{T}^{n} \times \mathbb{R}^{n}$ and let $\pi: \mathbb{T}^{n} \times \mathbb{R}^{n} \rightarrow \mathbb{R}^{n}$ be the second factor projection. The equations of motion of any Hamiltonian $H(q, p)$ with respect to $\omega_{\sigma}$ are given by

$$
\begin{aligned}
& \dot{q}=\nabla_{p} H, \\
& \dot{p}=J \nabla_{p} H-\nabla_{q} H,
\end{aligned}
$$


where $J: \mathbb{R}^{n} \rightarrow \mathbb{R}^{n}$ is the antisymmetric linear map determined by $\sigma(\cdot, \cdot)=\langle\cdot, J \cdot\rangle$. In particular, for the Hamiltonian $H(q, p)=(1 / 2)|p|^{2}$ we obtain

$$
\begin{aligned}
& \dot{q}=p, \\
& \dot{p}=J p .
\end{aligned}
$$

Split $\mathbb{R}^{n}$ orthogonally as $\mathbb{R}^{n}=\operatorname{Ker} J \oplus \operatorname{Im} J$ and let $P_{1}: \mathbb{R}^{n} \rightarrow \operatorname{Ker} J$ and $P_{2}: \mathbb{R}^{n} \rightarrow$ $\operatorname{Im} J$ be the corresponding orthogonal projections. The restriction of $J$ to $\operatorname{Im} J$ is invertible and we let $A:=\left(\left.J\right|_{\operatorname{Im} J}\right)^{-1}$. With this choice of $A$ we obtain a 1 -form $\alpha$ on $\operatorname{Im} J$ as in Lemma 6.2.

Let $f: \mathbb{T}^{n} \times \mathbb{R}^{n} \rightarrow \mathbb{T}^{n} \times \mathbb{R}^{n}$ be the map $f(q, p)=\left(q, P_{1}(p)\right)$ and let $v$ be the Liouville 1 -form in $T^{*} \mathbb{T}^{n}$. We claim that

$$
\lambda:=f^{*} v+\left(P_{2} \circ \pi\right)^{*} \alpha
$$

is a stabilizing 1 -form on $\Sigma_{k}$ for any $k>0$. Note that $d f_{(q, p)}\left(X_{H}\right)=(p, 0)$ and $d \pi_{(q, p)}\left(X_{H}\right)=J p$. Thus

$$
\lambda_{(q, p)}\left(X_{H}\right)=v_{\left(q, P_{1}(p)\right)}(p, 0)+\alpha_{P_{2}(p)}(J p)=\left|P_{1}(p)\right|^{2}+\frac{1}{2}\left|P_{2}(p)\right|^{2}
$$

which is always positive on $\Sigma_{k}$. On the other hand using Lemma 6.2 we obtain

$$
i_{X_{H}} d \lambda_{(q, p)}(x, y)=d \nu_{\left(q, P_{1}(p)\right)}\left((p, 0),\left(x, P_{1}(y)\right)\right)+\left\langle J p, A P_{2}(y)\right\rangle,
$$

where $(x, y)$ denotes a vector in $T_{(q, p)}\left(\mathbb{T}^{n} \times \mathbb{R}^{n}\right)$. Using the fact that $d v$ is the standard symplectic form on $T^{*} \mathbb{T}^{n}$ we obtain

$$
d v_{\left(q, P_{1}(p)\right)}\left((p, 0),\left(x, P_{1}(y)\right)\right)=-d H_{\left(q, P_{1}(p)\right)}\left(x, P_{1}(y)\right)=-\left\langle p, P_{1}(y)\right\rangle .
$$

Using the definition of $A$ the other term is

$$
\left\langle J p, A P_{2}(y)\right\rangle=-\left\langle p, J A P_{2}(y)\right\rangle=-\left\langle p, P_{2}(y)\right\rangle
$$

and hence

$$
i_{X_{H}} d \lambda_{(q, p)}(x, y)=-\langle p, y\rangle=0
$$

for $(x, y) \in T_{(q, p)} \Sigma_{k}$. This proves $\left.\left(i_{X_{H}} d \lambda\right)\right|_{\Sigma_{k}}=0$, so $\lambda$ is a stabilizing 1 -form for $\Sigma_{k}$.

Displaceability of $\Sigma_{k}$ is easy to see (cf [10, Theorem B] and the proof of Theorem 3.1 in [31]): Pick any $a \in \mathbb{R}^{n}$ with $J a \neq 0$ (this exists since $\sigma \neq 0$ ) and consider the Hamiltonian $h(q, p)=\langle a, p\rangle$. The corresponding Hamiltonian flow defined by

$$
\dot{q}=a, \quad \dot{p}=J a
$$

contains a translation in direction $J a$ and hence displaces every compact subset of $T^{*} \mathbb{T}^{n}$. 
In order to show that $\Sigma_{k}$ is tame we need to locate the contractible closed orbits. We can readily find the flow $\phi_{t}$ of $X_{H}$. Since $J$ is antisymmetric, the solution to $\dot{p}=J p$ is a 1 -parameter subgroup $t \mapsto e^{t J} \in \mathrm{SO}(n)$. We have

$$
\phi_{t}(q, p)=\left(\int_{0}^{t} e^{s J} p d s+q, e^{t J} p\right) .
$$

Write $p=p_{1}+p_{2}$, where $p_{1} \in \operatorname{Ker} J$ and $p_{2} \in \operatorname{Im} J$. Then $e^{s J} p=p_{1}+e^{s J} p_{2}$ and thus

$$
\int_{0}^{t} e^{s J} p d s=p_{1} t+\int_{0}^{t} e^{s J} p_{2} d s
$$

Let $\widehat{J}:=\left.J\right|_{\operatorname{Im} J}$. Then $e^{s \widehat{J}}=\left.e^{s J}\right|_{\operatorname{Im} J}$. But $\widehat{J}$ is invertible, so we may write

$$
\int_{0}^{t} e^{s J} p_{2} d s=\int_{0}^{t}(\widehat{J})^{-1} \frac{d}{d s}\left(e^{s \widehat{J}} p_{2}\right) d s=(\widehat{J})^{-1}\left(e^{t \widehat{J}}-I\right) p_{2} .
$$

Combining this with (51) and (52) we see that $(q, p) \in \Sigma_{k}$ gives rise to a closed contractible orbit of period $T$ if and only if $p_{1}=0, e^{T J} p_{2}=p_{2}$ and $\left|p_{2}\right|^{2}=2 k$.

Now, a primitive for $d p \wedge d q+\tau^{*} \sigma$ in $T^{*} \mathbb{R}^{n}$ is given by $p d q+\tau^{*} \beta$ where $\beta_{q}(a)=$ $(1 / 2)\langle q, J a\rangle$ (we use again Lemma 6.2). Thus, if $v$ is a closed contractible orbit in $\Sigma_{k}$, we have

$$
\Omega(v)=\int_{v}\left(p d q+\tau^{*} \beta\right)=2 k T+\int_{\tau(v)} \beta .
$$

We compute

$$
\int_{\tau(v)} \beta=\frac{1}{2} \int_{0}^{T}\left\langle(\widehat{J})^{-1}\left(e^{t \widehat{J}}-I\right) p_{2}, J e^{t \widehat{J}} p_{2}\right\rangle d t=-\frac{1}{2}\left|p_{2}\right|^{2} T=-k T,
$$

and therefore

$$
\Omega(v)=k T
$$

which clearly shows that $\Sigma_{k}$ is tame.

Remark It is instructive to see what the form $\lambda$ looks like in the following special cases:

- When $n=2$ and $\sigma=d q_{1} \wedge d q_{2}$, then

$$
\lambda=-\frac{1}{2}\left(p_{1} d p_{2}-p_{2} d p_{1}\right),
$$

- When $n=3$ and $\sigma=d q_{1} \wedge d q_{2}$, then

$$
\lambda=p_{3} d q_{3}-\frac{1}{2}\left(p_{1} d p_{2}-p_{2} d p_{1}\right) .
$$

Note that the Mañé critical value is infinite because a nonzero $\sigma$ has no bounded primitives in $\mathbb{R}^{n}$. 


\subsection{The Heisenberg group}

Let $G$ be the 3-dimensional Heisenberg group of matrices

$$
\left(\begin{array}{lll}
1 & x & z \\
0 & 1 & y \\
0 & 0 & 1
\end{array}\right),
$$

where $x, y, z \in \mathbb{R}$. If we identify $G$ with $\mathbb{R}^{3}$, then the product is

$$
(x, y, z) \star\left(x^{\prime}, y^{\prime}, z^{\prime}\right)=\left(x+x^{\prime}, y+y^{\prime}, z+z^{\prime}+x y^{\prime}\right) .
$$

For $\Gamma$ we take the lattice of those matrices with $x, y, z \in \mathbb{Z}$. However, the following discussion will hold for any cocompact lattice $\Gamma$. In fact, all lattices are isomorphic to the semidirect product $\mathbb{Z}^{2} \ltimes_{A} \mathbb{Z}$ where

$$
A=\left(\begin{array}{ll}
1 & k \\
0 & 1
\end{array}\right)
$$

for some positive integer $k$ [65].

The 1-forms $\alpha:=d x, \beta:=d y$ and $\gamma:=d z-x d y$ are left-invariant and provide a trivialization of $T^{*} G$ as $G \times \mathfrak{g}^{*}$. We let $\left(x, y, z, p_{\alpha}, p_{\beta}, p_{\gamma}\right)$ be the coordinates induced by this trivialization. Note that $\left(p_{\alpha}, p_{\beta}, p_{\gamma}\right)$ descend to coordinates on $T^{*}(\Gamma \backslash G)=$ $(\Gamma \backslash G) \times \mathfrak{g}^{*}$. The coordinates $\left(p_{\alpha}, p_{\beta}, p_{\gamma}\right)$ are related to the coordinates $\left(p_{x}, p_{y}, p_{z}\right)$ by $p_{\alpha}=p_{x}, p_{\beta}=p_{y}+x p_{z}$ and $p_{\gamma}=p_{z}$.

We consider the following left-invariant Hamiltonian $H$ (dual to a suitable left-invariant metric on $G$ ):

$$
2 H=p_{\alpha}^{2}+p_{\beta}^{2}+p_{\gamma}^{2}=p_{x}^{2}+\left(p_{y}+x p_{z}\right)^{2}+p_{z}^{2} .
$$

The magnetic field is given by the left-invariant 2 -form

$$
\sigma:=-d x \wedge d y
$$

Note that $\sigma$ descends to an exact 2-form on $\Gamma \backslash G$ since $\sigma=d \gamma$. Also observe that $2 H(\gamma) \equiv 1$, which implies that Mañé's critical value $c_{0}$ is $\leq 1 / 2$. Later on we will see that $c=c_{0}=1 / 2$.

The twisted symplectic form $\omega$ is

$$
\begin{aligned}
\omega & =d p_{x} \wedge d x+d p_{y} \wedge d y+d p_{z} \wedge d z-d x \wedge d y \\
& =d p_{\alpha} \wedge d x+d p_{\beta} \wedge d y+d p_{\gamma} \wedge d z-x d p_{\gamma} \wedge d y-\left(1+p_{\gamma}\right) d x \wedge d y
\end{aligned}
$$


The Hamiltonian vector field of a function $H$ with respect to $\omega$ is given by (where $H_{x}$ etc. denote partial derivatives)

$$
X_{H}= \begin{cases}\dot{x}=H_{p_{\alpha}}, & \dot{p}_{\alpha}=-H_{x}-\left(1+p_{\gamma}\right) H_{p_{\beta}}, \\ \dot{y}=H_{p_{\beta}}, & \dot{p}_{\beta}=-H_{y}+x H_{z}+\left(1+p_{\gamma}\right) H_{p_{\alpha}}, \\ \dot{z}=x H_{p_{\beta}}+H_{p_{\gamma}}, & \dot{p}_{\gamma}=-H_{z} .\end{cases}
$$

In particular, for the left-invariant Hamiltonian above we obtain

$$
X_{H}= \begin{cases}\dot{x}=p_{\alpha}, & \dot{p}_{\alpha}=-p_{\beta} p_{\gamma}-p_{\beta}, \\ \dot{y}=p_{\beta}, & \dot{p}_{\beta}=p_{\alpha} p_{\gamma}+p_{\alpha}, \\ \dot{z}=x p_{\beta}+p_{\gamma}, & \dot{p}_{\gamma}=0 .\end{cases}
$$

The nonzero Poisson brackets of the coordinate functions are $\left(X_{f}(\bullet)=\{f, \bullet\}\right)$

$$
\left\{p_{\alpha}, x\right\}=\left\{p_{\beta}, y\right\}=\left\{p_{\gamma}, z\right\}=1,\left\{p_{\beta}, z\right\}=x,\left\{p_{\alpha}, p_{\beta}\right\}=1+p_{\gamma} .
$$

Let the Liouville 1 -form be $v=p_{x} d x+p_{y} d y+p_{z} d z$ and let

$$
\psi:=v+\tau^{*} \gamma
$$

Clearly $d \psi=\omega$. We compute

$$
\begin{aligned}
\psi\left(X_{H}\right) & =p_{\alpha}^{2}+p_{\beta}\left(p_{\beta}-x p_{\gamma}\right)+p_{\gamma}\left(x p_{\beta}+p_{\gamma}\right)+p_{\gamma} \\
& =p_{\alpha}^{2}+p_{\beta}{ }^{2}+p_{\gamma}\left(p_{\gamma}+1\right)=2 H+p_{\gamma} .
\end{aligned}
$$

Let $\Sigma_{k}:=H^{-1}(k)$.

Lemma 6.4 The hypersurface $\Sigma_{k}$ is of contact type if and only if $k>1 / 2$.

Proof Equation (55) tells us right away that if $k>1 / 2$, then $\Sigma_{k}$ is of contact type with contact form $\psi$ since in this case

$$
\psi\left(X_{H}\right)=2 k+p_{\gamma} \geq 2 k-\sqrt{2 k}>0
$$

for every point in $\Sigma_{k}$.

An inspection of (54) reveals that $p_{\alpha}=p_{\beta}=y=0, p_{\gamma}=-\sqrt{2 k}, x=x_{0}, z(t)=$ $z_{0}-\sqrt{2 k} t$ give orbits of $X_{H}$ with energy $k$ which project to closed orbits in $T^{*}(\Gamma \backslash G)$. These closed orbits are in fact null-homologous since $\alpha$ and $\beta$ integrate to zero along them and these two 1 -forms span $H^{1}(\Gamma \backslash G, \mathbb{R})$. (This is because by a theorem of Nomizu [57] the de Rham cohomology ring of $\Gamma \backslash G$ is isomorphic to the Lie algebra cohomology of $\mathfrak{g}$ and the isomorphism is induced by the natural inclusion of 
left-invariant forms.) Let $\delta$ be one of these closed orbits. Using (55) we see that for $k \leq 1 / 2$,

$$
\int_{\delta} \psi=(2 k-\sqrt{2 k}) \tau \leq 0,
$$

where $\tau$ is the period of $\delta$. Now let $\mu$ be the Liouville measure on $\Sigma_{k}$. Again, using (55) we obtain

$$
\int_{\Sigma_{k}} \psi\left(X_{H}\right) d \mu=\int_{\Sigma_{k}}\left(2 k+p_{\gamma}\right) d \mu=2 k>0 .
$$

By Lemma 2.9 the Liouville measure is exact as a current, so (56), (57) and Corollary 2.8 ensure that $\Sigma_{k}$ cannot be of contact type. Alternatively, instead of the Liouville measure one may also take the distinguished null-homologous closed orbits with $p_{\alpha}=p_{\beta}=y=0, p_{\gamma}=\sqrt{2 k}, x=x_{0}$ and $z(t)=z_{0}+\sqrt{2 k} t$. The integral of $\psi$ along them has value $(2 k+\sqrt{2 k}) \tau>0$.

Lemma 6.5 The hypersurface $\Sigma_{k}$ is stable for $k<1 / 2$.

Proof Consider the following form on $\Sigma_{k}$ :

$$
\varphi:=\frac{p_{\alpha} d p_{\beta}-p_{\beta} d p_{\alpha}}{p_{\alpha}^{2}+p_{\beta}^{2}} .
$$

This form is smooth away from $p_{\gamma}= \pm \sqrt{2 k}$ and $d \varphi=0$. We look for stabilizing 1 -forms $\lambda$ of the form

$$
\lambda=f\left(p_{\gamma}\right) \psi+g\left(p_{\gamma}\right) \varphi,
$$

where $f$ and $g$ are smooth functions in $[-\sqrt{2 k}, \sqrt{2 k}]$ and $g$ vanishes in small neighbourhoods of the end points. Using $\psi\left(X_{H}\right)=2 k+p_{\gamma}$ and $\varphi\left(X_{H}\right)=1+p_{\gamma}$, the condition $\lambda\left(X_{H}\right)>0$ is equivalent to

$$
\left(2 k+p_{\gamma}\right) f\left(p_{\gamma}\right)+\left(1+p_{\gamma}\right) g\left(p_{\gamma}\right)>0,
$$

and the condition $i_{X_{H}} d \lambda=0$ is equivalent to

$$
\left(2 k+p_{\gamma}\right) f^{\prime}\left(p_{\gamma}\right)+\left(1+p_{\gamma}\right) g^{\prime}\left(p_{\gamma}\right)=0 .
$$

To see that we can always choose $f$ and $g$ satisfying (58) and (59) provided that $2 k<1$, take a nonnegative smooth function $r\left(p_{\gamma}\right)$ which vanishes near the end points of $[-\sqrt{2 k}, \sqrt{2 k}], r(-2 k)>0$ (note that $-\sqrt{2 k}<-2 k)$ and

$$
\int_{-\sqrt{2 k}}^{\sqrt{2 k}} \frac{2 k+p_{\gamma}}{1+p_{\gamma}} r\left(p_{\gamma}\right) d p_{\gamma}=0 .
$$


Note that this is always possible. Indeed as $r$ we could take $\left(1+p_{\gamma}\right) q\left(2 k+p_{\gamma}\right)$ where $q(t)$ is a smooth nonnegative even bump function with small support containing the origin. Now let

$$
\begin{aligned}
f\left(p_{\gamma}\right) & :=\int_{-2 k}^{p_{\gamma}} r(t) d t \\
g\left(p_{\gamma}\right) & :=-\int_{-\sqrt{2 k}}^{p_{\gamma}} \frac{2 k+t}{1+t} r(t) d t .
\end{aligned}
$$

Clearly (59) holds. With these choices, $f$ vanishes only at $-2 k$, is negative at the left of $-2 k$ and positive at the right of $-2 k$. Also $g \geq 0$, it vanishes near the end points and $g(-2 k)>0$, thus (58) also holds.

Lemma 6.6 The hypersurface $\Sigma_{k}$ is displaceable for $k<1 / 2$.

Proof Consider the Hamiltonian in $T^{*}(\Gamma \backslash G)$ given by $f=p_{\alpha}$. Clearly $p_{\gamma}$ does not change along the Hamiltonian flow of $f$ and $p_{\beta}$ changes according to

$$
\dot{p}_{\beta}=\left\{p_{\alpha}, p_{\beta}\right\}=1+p_{\gamma} .
$$

Thus if $k<1 / 2$ and we flow a point in $\Sigma_{k}$ along the Hamiltonian flow of $f$, we have $\dot{p_{\beta}} \geq 1-\sqrt{2 k}>0$. Thus the flow of $f$ will displace $\Sigma_{k}$ as desired.

Lemma 6.7 The hypersurface $\Sigma_{k}$ has closed contractible orbits if and only if $k<1 / 2$. If $k<1 / 2$ and $v \in X\left(\Sigma_{k}\right)$ then $\Omega(v)$ is a positive integer multiple of $2 \pi(1-\sqrt{1-2 k})>$ 0 and $\Sigma_{k}$ is tame.

Proof The equations in (54) show that $p_{\gamma}$ is a first integral (in fact it is the Casimir of $\left.\mathfrak{g}^{*}\right)$ and that if $p_{\gamma}+1 \neq 0$, then $p_{\alpha}$ and $p_{\beta}$ are trigonometric functions with period $T=2 \pi /\left(1+p_{\gamma}\right)$ and $p_{\alpha}^{2}+p_{\beta}^{2}=2 k-p_{\gamma}^{2}$. Since $p_{\alpha}$ and $p_{\beta}$ have zero integral along the period, $x$ and $y$ are also $T$-periodic functions. Hence we get a closed contractible orbit if and only if $z$ is periodic of period $T$ (or an integer multiple). To analyze this condition, abbreviate $\mu:=1+p_{\gamma} \neq 0$ and $a:=\sqrt{2 k-p_{\gamma}^{2}} \geq 0$. After time shift, the solutions of Hamilton's equations (54) are given by

$$
p_{\alpha}(t)=a \cos \mu t, \quad p_{\beta}(t)=a \sin \mu t, \quad p_{\gamma}=\mathrm{const}, \quad x(t)=x_{0}+\frac{a \sin \mu t}{\mu} .
$$

Again looking at (54), we see that periodicity of $z$ is equivalent to

$$
\int_{0}^{2 \pi /\left(1+p_{\gamma}\right)}\left(x(t) p_{\beta}(t)+p_{\gamma}\right) d t=0 .
$$


Evaluating the integral, we obtain the condition

$$
2 p_{\gamma}+\frac{2 k-p_{\gamma}{ }^{2}}{1+p_{\gamma}}=0 .
$$

If $k<1 / 2$, then $1+p_{\gamma}>0$ and there is a unique solution to (60) given by $p_{\gamma}=$ $\sqrt{1-2 k}-1 \in(-\sqrt{2 k}, 0)$, so we have contractible closed orbits.

If $k \geq 1 / 2$ it is easy to see that there are no such solutions. Indeed, this is clear if $1+p_{\gamma} \neq 0$ because Equation (60) has no real solutions for $k>1 / 2$ and only the solution $p_{\gamma}=-1$ for $k=1 / 2$. It remains to analyse the case $p_{\gamma}=-1$. It gives rise to a circle $p_{\alpha}{ }^{2}+p_{\beta}{ }^{2}=2 k-1$ of critical points for the Euler vector field (which degenerates to a point when $k=1 / 2$ ). Thus along solutions $p_{\alpha}, p_{\beta}, p_{\gamma}$ are constant, and from (54) we see that

$$
x(t)=x_{0}+p_{\alpha} t, \quad y(t)=y_{0}+p_{\beta} t
$$

are periodic if and only if $p_{\alpha}=p_{\beta}=0$ (so in particular $k=1 / 2$ ). But then $z(t)=$ $z_{0}+p_{\gamma} t$ is not periodic since $p_{\gamma} \neq 0$, so there are no contractible closed orbits for $k \geq 1 / 2$.

Since $d \psi=\omega$, to compute $\Omega(v)$ we just need to integrate $\psi$ along one of the closed orbits of $X_{H}$ described above. Since $\psi\left(X_{H}\right)=2 k+p_{\gamma}$ we deduce

$$
\Omega(v)=T\left(2 k+p_{\gamma}\right)=2 \pi(1-\sqrt{1-2 k})>0 .
$$

This also shows that $\Sigma_{k}$ is tame.

Remark It is not hard to show that for $k<1 / 2$, the closed contractible orbits form a Morse-Bott 4-dimensional submanifold of $\Sigma_{k}$ diffeomorphic to $(\Gamma \backslash G) \times S^{1}$.

Lemma 6.8 The Mañé critical values equal $c=c_{0}=1 / 2$.

Proof As noted above, $2 H(\gamma) \equiv 1$ implies $c_{0} \leq 1 / 2$. On the other hand, Lemma 6.4 and Lemma 5.3 imply $c_{0} \geq 1 / 2$, hence $c_{0}=1 / 2$. Since $\Gamma$ is nilpotent, it is amenable and $c=c_{0}$ by property (iv) in Section 5.3.

Lemma 6.9 The hypersurface $\Sigma_{1 / 2}$ is not stable.

Proof By Lemma 6.7, $\Sigma_{1 / 2}$ has no closed contractible orbits. By Lemma $6.6, \Sigma_{k}$ is displaceable for any $k<1 / 2$, hence Theorem 1.5 implies that $\Sigma_{1 / 2}$ is not stable. 


\section{3 $\operatorname{PSL}(2, \mathbb{R})$}

The magnetic flow discussed here also appears in [9]. It will be convenient for us to identify $\operatorname{PSL}(2, \mathbb{R})$ with $S \mathbb{H}^{2}$, the unit sphere bundle of the upper half plane with its usual metric of curvature -1

$$
\frac{d x^{2}+d y^{2}}{y^{2}} .
$$

We recall that the standard identification $\phi: S \mathbb{H}^{2} \rightarrow \operatorname{PSL}(2, \mathbb{R})$ is given as follows: $\phi(x, y, v)$ is the unique Möbius transformation $T$ (with real coefficients) such that $T(i)=x+i y$ and whose derivative at $i$ takes the tangent vector $(0,1)$ at $i$ to the tangent vector $v$ at $(x, y)$. In $S \mathbb{H}^{2}$ we consider coordinates $(x, y, \theta)$ and the vector fields

$$
\begin{aligned}
& X=y \cos \theta \frac{\partial}{\partial x}+y \sin \theta \frac{\partial}{\partial y}-\cos \theta \frac{\partial}{\partial \theta}, \\
& Y=-y \sin \theta \frac{\partial}{\partial x}+y \cos \theta \frac{\partial}{\partial y}+\sin \theta \frac{\partial}{\partial \theta}, \\
& V=\frac{\partial}{\partial \theta} .
\end{aligned}
$$

It is easy to check that these vector fields satisfy the bracket relations:

$$
[X, Y]=-V, \quad[V, X]=Y, \quad[V, Y]=-X,
$$

so they form the basis of a Lie algebra isomorphic to $\mathfrak{s l}(2, \mathbb{R})$. In fact under the map $\phi$ the vector fields $X, Y$ and $V$ correspond to the left invariant vector fields on $\operatorname{PSL}(2, \mathbb{R})$ whose values at the Lie algebra $\mathfrak{s l}(2, \mathbb{R})$ are

$$
X=\left(\begin{array}{cc}
1 / 2 & 0 \\
0 & -1 / 2
\end{array}\right), \quad Y=\left(\begin{array}{cc}
0 & -1 / 2 \\
-1 / 2 & 0
\end{array}\right), \quad V=\left(\begin{array}{cc}
0 & 1 / 2 \\
-1 / 2 & 0
\end{array}\right) .
$$

The dual coframe of left-invariant $1-$ forms $\{\alpha, \beta, \gamma\}$ is given by

$$
\begin{aligned}
& \alpha=\frac{\cos \theta d x+\sin \theta d y}{y}, \\
& \beta=\frac{-\sin \theta d x+\cos \theta d y}{y}, \\
& \gamma=\frac{d x}{y}+d \theta .
\end{aligned}
$$

Using this coframe we identify $T^{*} S \mathbb{H}^{2}$ with $S \mathbb{H}^{2} \times \mathfrak{s l}(2, \mathbb{R})^{*}$ and we obtain coordinates $\left(x, y, \theta, p_{\alpha}, p_{\beta}, p_{\gamma}\right)$. The left-invariant coordinates $\left(p_{\alpha}, p_{\beta}, p_{\gamma}\right)$ are related to 
$\left(p_{x}, p_{y}, p_{\theta}\right)$ by the formulas

$$
\begin{aligned}
& p_{\alpha}=\left(y p_{x}-p_{\theta}\right) \cos \theta+y p_{y} \sin \theta \\
& p_{\beta}=-\left(y p_{x}-p_{\theta}\right) \sin \theta+y p_{y} \cos \theta \\
& p_{\gamma}=p_{\theta}
\end{aligned}
$$

We will endow $S \mathbb{H}^{2}$ with its Sasaki metric. In this case this means the unique metric that makes the basis $\{X, Y, V\}$ an orthonormal frame at every point. Explicitly

$$
d s^{2}=\frac{1}{y^{2}}\left(\dot{x}^{2}+\dot{y}^{2}+(\dot{\theta} y+\dot{x})^{2}\right) .
$$

The Hamiltonian is

$$
2 H=\left(y p_{x}-p_{\theta}\right)^{2}+\left(y p_{y}\right)^{2}+p_{\theta}^{2}=p_{\alpha}^{2}+p_{\beta}^{2}+p_{\gamma}^{2} .
$$

The magnetic field is given by the left-invariant exact 2 -form

$$
\sigma:=d \gamma=\frac{d x \wedge d y}{y^{2}}
$$

The Hamiltonian vector field of $H$ with respect to the twisted symplectic form $\omega$ is given by

$$
X_{H}= \begin{cases}\dot{x}=y\left(y p_{x}-p_{\theta}\right), & \dot{p}_{x}=p_{y}, \\ \dot{y}=y^{2} p_{y}, & \dot{p}_{y}=\left(-y p_{x}+p_{\theta}\right)\left(p_{x}+1 / y\right)-y p_{y}^{2}, \\ \dot{\theta}=2 p_{\theta}-y p_{x}, & \dot{p}_{\theta}=0 .\end{cases}
$$

In terms of the left-invariant coordinates we get

$$
X_{H}= \begin{cases}\dot{x}=y\left(p_{\alpha} \cos \theta-p_{\beta} \sin \theta\right), & \dot{p}_{\alpha}=2 p_{\beta} p_{\gamma}+p_{\beta}, \\ \dot{y}=y\left(p_{\alpha} \sin \theta+p_{\beta} \cos \theta\right), & \dot{p}_{\beta}=-2 p_{\alpha} p_{\gamma}-p_{\alpha}, \\ \dot{\theta}=p_{\gamma}-p_{\alpha} \cos \theta+p_{\beta} \sin \theta, & \dot{p}_{\gamma}=0 .\end{cases}
$$

The Poisson brackets of the left-invariant coordinate functions are

$$
\left\{p_{\alpha}, p_{\beta}\right\}=-p_{\gamma}-1, \quad\left\{p_{\alpha}, p_{\gamma}\right\}=-p_{\beta}, \quad\left\{p_{\beta}, p_{\gamma}\right\}=p_{\alpha}
$$

Let the Liouville $1-$ form be $v=p_{x} d x+p_{y} d y+p_{\theta} d \theta$ and let

$$
\psi:=v+\tau^{*} \gamma
$$


Clearly $d \psi=\omega$. We compute

$$
\begin{aligned}
\psi\left(X_{H}\right) & =p_{x} y\left(y p_{x}-p_{\theta}\right)+y^{2} p_{y}^{2}+\left(2 p_{\theta}-y p_{x}\right)\left(p_{\theta}+1\right)+\left(y p_{x}-p_{\theta}\right) \\
& =2 H+p_{\theta}=2 H+p_{\gamma} .
\end{aligned}
$$

$\mathbb{H}^{2}$. We denote the quantities on the quotient $T^{*}(\Gamma \backslash \operatorname{PSL}(2, \mathbb{R}))$ by the same letters $H, \ldots$ Let $\Sigma_{k}:=H^{-1}(k)(k>0)$.

Lemma 6.10 The hypersurface $\Sigma_{k}$ is of contact type if and only if $k>1 / 2$.

Proof Equation (63) tells us right away that if $k>1 / 2$, then $\Sigma_{k}$ is of contact type with contact form $\psi$ since in this case

$$
\psi\left(X_{H}\right)=2 k+p_{\gamma} \geq 2 k-\sqrt{2 k}>0
$$

for every point in $\Sigma_{k}$. An inspection of (62) reveals that $p_{\alpha}=p_{\beta}=0, p_{\gamma}=-\sqrt{2 k}$, $x=x_{0}, y=y_{0}, \theta(t)=\theta_{0}-\sqrt{2 k} t$ give orbits of $X_{H}$ with energy $k$ which project to closed orbits in $T^{*}(\Gamma \backslash G)$. These closed orbits are in fact null-homologous. To see this note that $\Gamma \backslash \operatorname{PSL}(2, \mathbb{R})$ can be identified with the unit circle bundle of the closed Riemann surface $\Gamma \backslash \mathbb{H}^{2}$. The closed orbits of $X_{H}$ under consideration, when projected to $\Gamma \backslash \operatorname{PSL}(2, \mathbb{R})$, are precisely the unit circles in $S\left(\Gamma \backslash \mathbb{H}^{2}\right)$. But these circles are null-homologous. Indeed, it is well known (and it follows from the Gysin sequence of the circle bundle), that the foot-point projection map $S\left(\Gamma \backslash \mathbb{H}^{2}\right) \mapsto \Gamma \backslash \mathbb{H}^{2}$ induces an isomorphism $H^{1}\left(\Gamma \backslash \mathbb{H}^{2}, \mathbb{R}\right) \cong H^{1}\left(S\left(\Gamma \backslash \mathbb{H}^{2}\right), \mathbb{R}\right)$.

Let $\delta$ be one of these distinguished null-homologous closed orbits of $X_{H}$ and let $\tau$ be its period. Using (63) we see that for $k \leq 1 / 2$,

$$
\int_{\delta} \psi=(2 k-\sqrt{2 k}) \tau \leq 0 .
$$

Now let $\mu$ be the Liouville measure on $\Sigma_{k}$. Again, using (63) we obtain

$$
\int_{\Sigma_{k}} \psi\left(X_{H}\right) d \mu=\int_{\Sigma_{k}}\left(2 k+p_{\gamma}\right) d \mu=2 k>0 .
$$

By Lemma 2.9 the Liouville measure is exact as a current, so (64), (65) and Corollary 2.8 ensure that $\Sigma_{k}$ cannot be of contact type. Alternatively, instead of the Liouville measure one may also take the distinguished null-homologous closed orbits with $p_{\alpha}=p_{\beta}=0, p_{\gamma}=\sqrt{2 k}, x=x_{0}, y=y_{0}$ and $\theta(t)=\theta_{0}+\sqrt{2 k} t$. The integral of $\psi$ along them has value $(2 k+\sqrt{2 k}) \tau>0$.

We now prove that in this example we have a gap between $c$ and $c_{0}$ of size $1 / 4$. 
Lemma 6.11 The strict Mañé critical value equals $c_{0}=1 / 2$, but the Mañé critical value equals $c=1 / 4$.

Proof Note that $2 H(\gamma)=1$ and thus $c_{0} \leq 1 / 2$. If $c_{0}<1 / 2$, then by Lemma 5.3 we would have energy levels $\Sigma_{k}$ with $k<1 / 2$ which are of contact type, which is impossible by Lemma 6.10. Thus $c_{0}=1 / 2$.

Now consider the 1 -form

$$
\delta:=\frac{d x}{y}+\frac{d \theta}{2} .
$$

Clearly $d \delta=d \gamma$ in $S \mathbb{H}^{2}$ and $2 H(\delta)=1 / 2$. (Note that $\delta$ is not left-invariant.) Thus $c \leq 1 / 4$. The fact that $c=1 / 4$ is most easily seen as follows. Consider the Lagrangian $L$ in $S \mathbb{H}^{2}$ determined by $\delta$, namely

$$
L=\frac{1}{2 y^{2}}\left(\dot{x}^{2}+\dot{y}^{2}+(\dot{\theta} y+\dot{x})^{2}\right)+\frac{\dot{x}}{y}+\frac{\dot{\theta}}{2} .
$$

Let $C_{r}$ be the curve in $\mathbb{H}^{2}$ given by the boundary of a geodesic disk $D_{r}$ of radius $r$. We parametrize $C_{r}$ so that it has hyperbolic speed equal to $\sqrt{2 k}$ and it is oriented clockwise. Let $\ell_{r}=2 \pi \sinh r$ be its hyperbolic length. If we let $(x(t), y(t))$ be the chosen parametrization for $C_{r}$, let $B_{r}$ be the closed curve given by $\left[0, \ell_{r} / \sqrt{2 k}\right] \ni t \mapsto$ $(x(t) / \sqrt{2}, y(t))$. By taking $\theta$ constant, we may see $B_{r}$ as a closed contractible curve in $S \mathbb{H}^{2}$. We will compute the action $A_{L+k}\left(B_{r}\right)$ and show that for any $k<1 / 4$, there is $r$ such that $A_{L+k}\left(B_{r}\right)<0$. This implies $c=1 / 4$ (cf Section 5). Indeed we have

$$
\begin{aligned}
A_{L+k}\left(B_{r}\right) & =\sqrt{2 k} \ell_{r}+\frac{1}{\sqrt{2}} \int_{0}^{\ell_{r} / \sqrt{2 k}} \frac{\dot{x}}{y} d t \\
& =\sqrt{2 k} \ell_{r}-\frac{1}{\sqrt{2}} \int_{D_{r}} \frac{d x \wedge d y}{y^{2}} \\
& =2 \pi\left(\sqrt{2 k} \sinh r-\frac{\cosh r-1}{\sqrt{2}}\right) .
\end{aligned}
$$

The last expression shows that if $k<1 / 4$, then $A_{L+k}\left(B_{r}\right) \rightarrow-\infty$ as $r \rightarrow \infty$.

Lemma 6.12 The hypersurface $\Sigma_{k}$ is stable for $k<1 / 4$ and $k \in(1 / 4,1 / 2)$.

Proof Consider the following form on $\Sigma_{k}$ :

$$
\varphi:=\frac{-p_{\alpha} d p_{\beta}+p_{\beta} d p_{\alpha}}{p_{\alpha}^{2}+p_{\beta}^{2}} .
$$


This form is smooth away from $p_{\gamma}= \pm \sqrt{2 k}$ and $d \varphi=0$. As in the case of the Heisenberg group, we look for stabilizing 1 -forms $\lambda$ of the form

$$
\lambda=f\left(p_{\gamma}\right) \psi+g\left(p_{\gamma}\right) \varphi,
$$

where $f$ and $g$ are smooth functions in $[-\sqrt{2 k}, \sqrt{2 k}]$ and $g$ vanishes in neighbourhoods of the end points. Using $\psi\left(X_{H}\right)=2 k+p_{\gamma}$ and $\varphi\left(X_{H}\right)=1+2 p_{\gamma}$, the condition $\lambda\left(X_{H}\right)>0$ is equivalent to

$$
\left(2 k+p_{\gamma}\right) f\left(p_{\gamma}\right)+\left(1+2 p_{\gamma}\right) g\left(p_{\gamma}\right)>0,
$$

and the condition $i_{X_{H}} d \lambda=0$ is equivalent to

$$
\left(2 k+p_{\gamma}\right) f^{\prime}\left(p_{\gamma}\right)+\left(1+2 p_{\gamma}\right) g^{\prime}\left(p_{\gamma}\right)=0 .
$$

To see that we can always choose $f$ and $g$ satisfying (66) and (67) provided that $k<1 / 4$ or $k \in(1 / 4,1 / 2)$ we proceed as in the case of the Heisenberg group, but we will need some minor adjustments.

Case $\boldsymbol{k}<\mathbf{1 / 4}$ Here $-\sqrt{2 k}<-1 / 2<-2 k$.

Take a nonnegative smooth function $r\left(p_{\gamma}\right)$ which vanishes outside a small neighbourhood of $-2 k$ which excludes $-1 / 2$ such that $r(-2 k)>0$ and

$$
\int_{-\sqrt{2 k}}^{\sqrt{2 k}} \frac{2 k+p_{\gamma}}{1+2 p_{\gamma}} r\left(p_{\gamma}\right) d p_{\gamma}=0 .
$$

Note that this is always possible. Indeed as $r$ we could take $\left(1+2 p_{\gamma}\right) q\left(2 k+p_{\gamma}\right)$ where $q(t)$ is a smooth nonnegative even bump function with small support containing the origin. Now let

$$
\begin{aligned}
f\left(p_{\gamma}\right) & :=\int_{-2 k}^{p_{\gamma}} r(t) d t \\
g\left(p_{\gamma}\right) & :=-\int_{-\sqrt{2 k}}^{p_{\gamma}} \frac{2 k+t}{1+2 t} r(t) d t .
\end{aligned}
$$

Clearly (67) holds. With these choices, $f$ vanishes only at $-2 k$, is negative at the left of $-2 k$ and positive at the right of $-2 k$. Also $g \geq 0$, it vanishes outside a small neighbourhood of $-2 k$ and $g(-2 k)>0$, thus (66) also holds.

Case $k \in(1 / 4,1 / 2)$ Here $-\sqrt{2 k}<-2 k<-1 / 2$. As in the previous case, take a nonnegative smooth function $r\left(p_{\gamma}\right)$ which vanishes outside a small neighbourhood 
of $-2 k$ which excludes $-1 / 2$ such that $r(-2 k)>0$ and

$$
\int_{-\sqrt{2 k}}^{\sqrt{2 k}} \frac{2 k+p_{\gamma}}{1+2 p_{\gamma}} r\left(p_{\gamma}\right) d p_{\gamma}=0 .
$$

Also let

$$
\begin{aligned}
f\left(p_{\gamma}\right) & :=\int_{-2 k}^{p_{\gamma}} r(t) d t, \\
g\left(p_{\gamma}\right) & :=-\int_{-\sqrt{2 k}}^{p_{\gamma}} \frac{2 k+t}{1+2 t} r(t) d t .
\end{aligned}
$$

Clearly (67) holds. With these choices, $f$ vanishes only at $-2 k$, is negative at the left of $-2 k$ and positive at the right of $-2 k$. But now $g \leq 0$, it vanishes outside a small neighbourhood of $-2 k$ and $g(-2 k)<0$, thus (66) also holds since now $1+2 p_{\gamma}$ is negative in the small neighbourhood of $-2 k$.

Lemma 6.13 The hypersurface $\Sigma_{k}$ is displaceable for $k<1 / 4$.

Proof Consider the Hamiltonian in $T^{*}(\Gamma \backslash G)$ given by $f=p_{\beta}$. Clearly $p_{\beta}$ does not change along the Hamiltonian flow of $f$ and $p_{\alpha}$ and $p_{\gamma}$ change according to

$$
\begin{aligned}
& \dot{p_{\alpha}}=\left\{p_{\beta}, p_{\alpha}\right\}=p_{\gamma}+1, \\
& \dot{p_{\gamma}}=\left\{p_{\beta}, p_{\gamma}\right\}=p_{\alpha} .
\end{aligned}
$$

These equations are easy to solve and one finds that

$$
2 p_{\alpha}(t)=\left(p_{\alpha}(0)+p_{\gamma}(0)+1\right) e^{t}+\left(p_{\alpha}(0)-p_{\gamma}(0)-1\right) e^{-t} .
$$

But if $k<1 / 4$ and $\left(p_{\alpha}(0)\right)^{2}+\left(p_{\beta}(0)\right)^{2}+\left(p_{\gamma}(0)\right)^{2}=2 k$, then

$$
p_{\alpha}(0)+p_{\gamma}(0)+1 \geq 1-2 \sqrt{k}>0,
$$

and the flow of $f$ will displace $\Sigma_{k}$ as desired.

Closed contractible orbits and entropy We now will study dynamical properties of the magnetic flow. For this purpose it is better to revert to $\operatorname{PSL}(2, \mathbb{R})$ and use the group structure.

Equation (62) gives the Euler equations in $\mathfrak{s l}(2, \mathbb{R})^{*}$. These are easily solved and after a time shift one obtains

$$
p_{\alpha}=A \cos \mu t, \quad p_{\beta}=A \sin \mu t, \quad p_{\gamma}=C,
$$


where $\mu:=-1-2 C, A^{2}+C^{2}=2 k$ and $k$ is the value of the energy. This curve $b(t)$ in $\mathfrak{s l}(2, \mathbb{R})^{*}$ gives rise to a curve $a(t)$ in $\mathfrak{s l}(2, \mathbb{R})$ via the Legendre transform of $H$ :

$$
\begin{aligned}
a(t) & =A \cos \mu t X+A \sin \mu t Y+C V \\
& =A \cos \mu t\left(\begin{array}{cc}
1 / 2 & 0 \\
0 & -1 / 2
\end{array}\right)+A \sin \mu t\left(\begin{array}{cc}
0 & -1 / 2 \\
-1 / 2 & 0
\end{array}\right)+C\left(\begin{array}{cc}
0 & 1 / 2 \\
-1 / 2 & 0
\end{array}\right) \\
& =\frac{A}{2}\left(\begin{array}{cc}
\cos \mu t & -\sin \mu t \\
-\sin \mu t & -\cos \mu t
\end{array}\right)+\frac{C}{2}\left(\begin{array}{cc}
0 & 1 \\
-1 & 0
\end{array}\right) .
\end{aligned}
$$

Let us consider the loop of matrices in $\mathrm{SO}(2)$ given by

$$
Q(t):=\left(\begin{array}{cc}
\cos (\mu t / 2) & \sin (\mu t / 2) \\
-\sin (\mu t / 2) & \cos (\mu t / 2)
\end{array}\right)
$$

Clearly $Q(t)=e^{t R}$ where

$$
R=\left(\begin{array}{cc}
0 & \mu / 2 \\
-\mu / 2 & 0
\end{array}\right)
$$

Then we may write $a(t)$ as

$$
a(t)=Q(t)\left(\begin{array}{cc}
A / 2 & 0 \\
0 & -A / 2
\end{array}\right) Q^{*}(t)+\frac{C}{2}\left(\begin{array}{cc}
0 & 1 \\
-1 & 0
\end{array}\right),
$$

where $Q^{*}(t)$ denotes the transpose of $Q(t)$. Since our system is left-invariant, in order to find the solution of $X_{H}$ through a point $(q, p) \in \operatorname{PSL}(2, \mathbb{R}) \times \mathfrak{s l}(2, \mathbb{R})^{*}$ with $H(p)=k$ and $p=(A, 0, C)$ we may proceed as follows (cf [5, Appendix 2] or [2, Section 4.4]). We compute $a(t)$ and then we solve the matrix differential equation in $\operatorname{PSL}(2, \mathbb{R})$ given by

$$
\dot{g}(t)=g(t) a(t), \quad g(0)=I .
$$

The desired solution curve is $(q g(t), b(t))$. To solve the matrix differential equation we make the change of variables

$$
h(t):=g(t) Q(t) .
$$

A calculation using (68) shows that $h$ satisfies

$$
\dot{h}(t)=h(t) d,
$$

where $d$ is the constant matrix

$$
d:=\frac{1}{2}\left(\begin{array}{cc}
A & C+\mu \\
-(C+\mu) & -A
\end{array}\right)=\frac{1}{2}\left(\begin{array}{cc}
A & -1-C \\
1+C & -A
\end{array}\right) .
$$


Therefore we have obtained the following explicit formula for our magnetic flow $\phi_{t}$ :

$$
\phi_{t}(q, p)=\left(q e^{t d} Q^{*}(t), b(t)\right) .
$$

From this equation we shall derive various dynamical consequences. We will say that the matrix $d \in \mathfrak{s l}(2, \mathbb{R})$ is elliptic if $(1+C)^{2}>A^{2}$, parabolic if $(1+C)^{2}=A^{2}$ and hyperbolic if $(1+C)^{2}<A^{2}$.

Recall from Section 3 the definition of tameness and the $\omega$-energy

$$
\Omega: X\left(\Sigma_{k}\right) \rightarrow \mathbb{R}, \quad v \mapsto \int \bar{v}^{*} \omega
$$

Lemma 6.14 The hypersurface $\Sigma_{k}$ has closed contractible orbits if and only if $k<1 / 4$. If $k<1 / 4$ and $v \in X\left(\Sigma_{k}\right)$ then $\Omega(v)$ is a positive integer multiple of $\pi(1-\sqrt{1-4 k})>0$ and $\Sigma_{k}$ is tame.

Proof Before embarking into the proof we make some preliminary remarks about $\operatorname{PSL}(2, \mathbb{R})$. A matrix $d \in \mathfrak{s l}(2, \mathbb{R})$ satisfies $e^{d}= \pm I$ if and only if $\operatorname{det} d>0$ (ie $d$ is elliptic) and $\sqrt{\operatorname{det} d} / \pi$ is a positive integer. Let us call $\tau:=\pi / \sqrt{\operatorname{det} d}$ the period of $d$. It has the property of being the smallest positive real number $t$ for which $e^{t d}= \pm I$ (and in fact equal to $-I$ ). The fundamental group of $\operatorname{PSL}(2, \mathbb{R})=S \mathbb{H}^{2}$ is of course $\mathbb{Z}$ and is generated by the loop $[0, \tau] \ni t \mapsto e^{t d}$ of any elliptic element $d$. Any two such loops determined by elements $d_{1}$ and $d_{2}$ will be homotopic if and only if $d_{1}$ and $d_{2}$ belong to the same side (ie connected component) of the solid cone $\operatorname{det} d>0$ in the Lie algebra $\mathfrak{s t}(2, \mathbb{R})$. If they belong to opposite sides of the cone, they will have opposite homotopy classes.

Suppose now that we look for a closed contractible orbit on $\Sigma_{k}$ with $\mu \neq 0$. For $b(t)$ to be periodic it must have period $T:=2 \pi l /|\mu|$ where $l$ is a positive integer. Using (69) we see that

$$
\phi_{T}(q, p)=\left(q e^{T d}, p\right)=(q, p)
$$

and this happens if and only if the matrix $d$ is elliptic and $e^{T d}= \pm I$ (recall that we are working in $\operatorname{PSL}(2, \mathbb{R}))$. The last equality forces $T=m \tau$, where $m$ is a positive integer and $\tau$ is the period of $d$ as defined above. In order for this closed orbit to be contractible in $\Sigma_{k}$ we need the loop

$$
[0, T] \ni t \mapsto e^{t d} e^{-t R}
$$

to be contractible in $\operatorname{PSL}(2, \mathbb{R})$. But the homotopy class of this loop is $m[d]-l[R]$, where $[d]$ and $[R]$ denote the homotopy classes associated to $d$ and $R$ respectively. Since $m$ and $l$ are positive and $[d]= \pm[R]$, the loop will be contractible if and only if $m=l$ and $[d]=[R]$ (ie $d$ and $R$ must belong to the same side of the cone $\operatorname{det} d>0$ ). 
Hence $2|\mu|=1 / \sqrt{\operatorname{det} d}$ which translates into $2 C^{2}+2 C=-2 k$. Since $d$ is elliptic we must have $(1+C)^{2}>A^{2}=2 k-C^{2}$ which implies $k<1 / 4$ as desired.

The quadratic equation $2 C^{2}+2 C=-2 k$ gives the following values for $C$ and $\mu$ :

$$
2 C=-1 \pm \sqrt{1-4 k}, \mu=\mp \sqrt{1-4 k} .
$$

However, the values $2 C=-1-\sqrt{1-4 k}, \mu=\sqrt{1-4 k}$ give rise to elements $d$ and $R$ such that $[d]=-[R]$. Hence the unique values of $C$ and $\mu$ for which we obtain closed contractible orbits are

$$
2 C=-1+\sqrt{1-4 k}, \quad \mu=-\sqrt{1-4 k} .
$$

Finally, note that if we are at a $p$ with $\mu=0$, then the flow is just $\phi_{t}(q, p)=\left(q e^{t d}, p\right)$ and even though the orbits are all closed, they are clearly not contractible.

Since $d \psi=\omega$, to compute $\Omega(v)$ for a contractible closed orbit $v$ on $\Sigma_{k}$ we just need to integrate $\psi$ along one of the closed orbits of $\phi_{t}$ described above. Since $\psi\left(X_{H}\right)=2 k+C$ we deduce

$$
\Omega(v)=T(2 k+C)=\pi l(1-\sqrt{1-4 k})>0 .
$$

This also shows that $\Sigma_{k}$ is tame.

Lemma 6.15 The topological entropy of $\phi_{t}$ on $\Sigma_{k}$ is zero if and only if $k \leq 1 / 4$.

Proof Recall that by the variational principle for topological entropy and Ruelle's inequality, the topological entropy vanishes if all the Lyapunov exponents are zero. Recall also that by Pesin's formula, the topological entropy is at least the integral of the sum of the positive Lyapunov exponents with respect to the Liouville measure [42]. Inspection of (69) shows that all the Lyapunov exponents are zero if all possible $d$ 's are elliptic or parabolic. It also shows that as soon as there are $d$ 's which are hyperbolic, there will be a positive measure set (with respect to the Liouville measure) with positive Lyapunov exponents. Hence the topological entropy of the flow is zero if and only if all possible $d$ 's are elliptic or parabolic. This will happen if and only if $2 C^{2}+2 C+1 \geq 2 k$ for all $C \in[-\sqrt{2 k}, \sqrt{2 k}]$. It is easy to see that this is equivalent to saying that $k \leq 1 / 4$.

Lemma 6.16 The hypersurfaces $\Sigma_{1 / 2}$ and $\Sigma_{1 / 4}$ are not stable.

Proof By Lemma $6.14, \Sigma_{1 / 4}$ has no closed contractible orbits. By Lemma $6.13, \Sigma_{k}$ is displaceable for any $k<1 / 4$, hence Theorem 1.5 implies that $\Sigma_{1 / 4}$ is not stable. Alternatively, we could say that $\Sigma_{1 / 4}$ contains a copy of the horocycle flow (arising when $\mu=0$ ) and thus it cannot be stable. 
The proof that $\Sigma_{1 / 2}$ is not stable is a bit more involved and we need some preliminary observations.

Lemma 6.17 Let $N$ be a closed manifold with a nonzero geodesible vector field $F$. Suppose $G$ is a compact Lie group acting on $N$ and leaving $F$ invariant. Then there exists a $G$-invariant 1 -form which stabilizes $F$.

Proof Let $\mu$ be the Haar probability measure and $\varphi_{g}$ the diffeomorphism on $N$ determined by $g \in G$. Let $\lambda$ be a stabilizing 1 -form for $F$ and set

$$
\tilde{\lambda}:=\int_{G} \varphi_{g}^{*} \lambda d \mu(g)
$$

It is straightforward to check that $\tilde{\lambda}$ is $G$-invariant and that it stabilizes $F$.

Consider the flow of $V$ on $\Gamma \backslash \operatorname{PSL}(2, \mathbb{R})$; it is simply given by the right action of $e^{t V}$. Its lift to $T^{*}(\Gamma \backslash \operatorname{PSL}(2, \mathbb{R}))=\Gamma \backslash \operatorname{PSL}(2, \mathbb{R}) \times \mathfrak{s l}(2, \mathbb{R})^{*}$ is given by

$$
(q, p) \mapsto\left(q e^{t V}, \operatorname{Ad}_{e^{t V}}^{*}(p)\right) .
$$

This gives an action of $S^{1}$ that leaves $H, \tau^{*} \gamma$ and $d \psi$ invariant, so $S^{1}$ acts on $\left(T^{*}(\Gamma \backslash \operatorname{PSL}(2, \mathbb{R})), \omega\right)$ by Hamiltonian transformations and it has moment map $p_{\gamma}$. We denote the vector field of this $S^{1}$ action by $V^{*}$.

Let $\Sigma_{1 / 2, C} \subset \Sigma_{1 / 2}$ be given by $p_{\gamma}=C$ and $C$ is constant (energy-momentum reduction). Clearly $S^{1}$ leaves $\Sigma_{1 / 2, C}$ invariant and, $X_{H}$ and $V^{*}$ are linearly independent except when $C= \pm 1$.

Suppose $\Sigma_{1 / 2}$ is stable. By Lemma 6.17 we may take an $S^{1}$-invariant stabilizing 1 -form $\lambda$. Consider the restriction of both $\lambda$ and $\psi$ to the 4 -manifold $\Sigma_{1 / 2, C}$ for $C>-1$ close to -1 . The forms $d \lambda$ and $d \psi=\omega$ are both annihilated by $X_{H}$. The form $d \psi$ is annihilated by $V^{*}$ and we claim that $d \lambda$ is also annihilated by $V^{*}$. To see this, it suffices to show that $\lambda\left(V^{*}\right)$ is a constant function since $\lambda$ is $S^{1}$-invariant. Observe that the function $\lambda\left(V^{*}\right)$ is invariant under both $S^{1}$ and the flow of $X_{H}$ so it descends to a function on $\Sigma_{1 / 2, C} / S^{1}=\Gamma \backslash \operatorname{PSL}(2, \mathbb{R})$ which is invariant under the reduced flow of $X_{H}$. However, this reduced flow is given by the right action of $e^{t d}$. This follows from (69) and (70) (note that $b(t)=\operatorname{Ad}_{e^{-t \mu V}}^{*}(p)$ ). Since $d$ is hyperbolic for $-1<C<0$, the right action of $e^{t d}$ is Anosov and thus $\lambda\left(V^{*}\right)$ must be a constant. Hence, the forms $d \lambda$ and $d \psi=\omega$ are both annihilated by $X_{H}$ and $V^{*}$. Since $d \psi$ is nondegenerate in a transverse plane to the span of $X_{H}$ and $V^{*}$ we deduce that there exists a smooth function $f: \Sigma_{1 / 2, C} \rightarrow \mathbb{R}$ such that on $\Sigma_{1 / 2, C}$ we have

$$
d \lambda=f d \psi
$$


The function $f$ is invariant under both $S^{1}$ and the flow of $X_{H}$ so it also descends to a function on $\Sigma_{1 / 2, C} / S^{1}=\Gamma \backslash \operatorname{PSL}(2, \mathbb{R})$ which is invariant under the reduced flow of $X_{H}$ and as above, we deduce that $f$ must be a constant. If we now let $C$ vary close to -1 we get $f=f(C)$ and $d \lambda=f(C) d \psi$. We wish to derive a contradiction from this.

We proceed as follows. On $\Sigma_{1 / 2}=\Gamma \backslash \operatorname{PSL}(2, \mathbb{R}) \times S^{2}$ we let $\tilde{X}:=(X, 0)$ and $\tilde{Y}:=(Y, 0)$. These vectors are of course tangent to $\Sigma_{1 / 2, C}$ for every $C$. A simple calculation shows that $d \psi(\tilde{X}, \tilde{Y})=d \nu(\tilde{X}, \tilde{Y})+\tau^{*} d \gamma(\tilde{X}, \tilde{Y})=0+1=1$ and thus $f(C)=d \lambda(\tilde{X}, \tilde{Y})$. We now let $C \rightarrow-1$ and we may suppose that $f(C) \rightarrow a$ for some $a \in \mathbb{R}$. On $\Sigma_{1 / 2,-1}=\Gamma \backslash \operatorname{PSL}(2, \mathbb{R}) \times\{(0,0,-1)\}$ we have

$$
d \lambda=a d \psi \text {. }
$$

The 1 -form $\lambda-a \psi$ is closed and $(\lambda-a \psi)\left(X_{H}\right)=\lambda\left(X_{H}\right)>0$ since $\psi\left(X_{H}\right)=0$ on $\Sigma_{1 / 2,-1}$. But this is impossible since the Hamiltonian flow on $\Sigma_{1 / 2,-1}$ is given by the circle action

$$
\phi_{t}(q, p)=\left(q Q^{*}(t), p\right),
$$

which is completely periodic with all closed orbits homologous to zero. Thus $\Sigma_{1 / 2}$ cannot be stable and Lemma 6.16 is proved.

\subsection{Sol-manifolds}

Let $G=$ Sol be the semidirect product of $\mathbb{R}^{2}$ with $\mathbb{R}$, with coordinates $\left(y_{0}, y_{1}, u\right)$ and multiplication

$$
\left(y_{0}, y_{1}, u\right) \star\left(y_{0}^{\prime}, y_{1}^{\prime}, u^{\prime}\right)=\left(y_{0}+e^{u} y_{0}^{\prime}, y_{1}+e^{-u} y_{1}^{\prime}, u+u^{\prime}\right) .
$$

The map $\left(y_{0}, y_{1}, u\right) \mapsto u$ is the epimorphism Sol $\rightarrow \mathbb{R}$ whose kernel is the normal subgroup $\mathbb{R}^{2}$. The group Sol is isomorphic to the matrix group

$$
\left(\begin{array}{ccc}
e^{u} & 0 & y_{0} \\
0 & e^{-u} & y_{1} \\
0 & 0 & 1
\end{array}\right)
$$

It is not difficult to see that Sol admits cocompact lattices. Let $A \in S L(2, \mathbb{Z})$ be such that there is $P \in \mathrm{GL}(2, \mathbb{R})$ with

$$
P A P^{-1}=\left(\begin{array}{cc}
\lambda & 0 \\
0 & 1 / \lambda
\end{array}\right)
$$

and $\lambda>1$. There is an injective homomorphism

$$
\mathbb{Z}^{2} \ltimes_{A} \mathbb{Z} \hookrightarrow \text { Sol }
$$


given by $(m, n, l) \mapsto(P(m, n),(\log \lambda) l)$ which defines a cocompact lattice $\Gamma$ in Sol. The closed 3-manifold $\Gamma \backslash$ Sol is a 2-torus bundle over the circle with hyperbolic gluing map $A$.

If we denote by $p_{u}, p_{y_{0}}$ and $p_{y_{1}}$ the momenta that are canonically conjugate to $u$, $y_{0}$ and $y_{1}$ respectively, then the functions

$$
\begin{aligned}
\alpha_{0} & =e^{u} p_{y_{0}}, \\
\alpha_{1} & =e^{-u} p_{y_{1}}, \\
v & =p_{u}
\end{aligned}
$$

are left-invariant functions on $T^{*}$ Sol. The closed 2 -form

$$
\sigma=-d y_{0} \wedge d y_{1}
$$

is also left-invariant, and we consider the twisted symplectic form

$$
\omega_{\sigma}=d p_{u} \wedge d u+d p_{y_{0}} \wedge d y_{0}+d p_{y_{1}} \wedge d y_{1}-d y_{0} \wedge d y_{1}
$$

The Casimir of $\mathfrak{g}^{*}$ is $f=v+\alpha_{0} \alpha_{1}$ and the left-invariant Hamiltonian is given by $2 H:=\alpha_{0}^{2}+\alpha_{1}^{2}+v^{2}$. An easy calculation shows that the vector field of the magnetic flow is

$$
X_{H}=\left(e^{u} \alpha_{0}, e^{-u} \alpha_{1}, v,-\alpha_{1}+v \alpha_{0}, \alpha_{0}-v \alpha_{1}, \alpha_{1}^{2}-\alpha_{0}^{2}\right) .
$$

The dynamics of the magnetic flow is very interesting and was investigated in [10]. It turns out that all energy levels have positive Liouville entropy and they all carry closed contractible orbits. The magnetic field is nonexact and is a generator of $H^{2}(\Gamma \backslash$ Sol, $\mathbb{R})$. Since the lattices are solvable there are no bounded primitives for $\sigma$ in Sol and the Mañé critical value is $\infty$.

Proposition 6.18 The hypersurface $\Sigma_{k}$ is stable and displaceable for any $k>0$.

\section{Proof Let}

$$
\lambda:=f d u+\frac{1}{2}\left(\alpha_{0} d \alpha_{1}-\alpha_{1} d \alpha_{0}\right) .
$$

Since all objects involved in the definition of $\lambda$ are left-invariant, $\lambda$ descends to compact quotients of Sol. A calculation using that $d f\left(X_{H}\right)=0$ shows that $i_{X_{H}} d \lambda=0$. One also sees that

$$
\lambda\left(X_{H}\right)=k+\frac{v^{2}}{2}>0,
$$

and thus $\lambda$ stabilizes $\Sigma_{k}$. The proof that $\Sigma_{k}$ is displaceable can be found in [10] and is similar to the proof of Lemma 6.6. 


\subsection{The case of $\sigma$ symplectic}

In this subsection we study the special case in which the closed left-invariant 2-form $\sigma$ is symplectic and we show that low energy levels are always stable.

Proposition 6.19 Assume that $\sigma$ is symplectic and let $H(\mu)=|\mu|^{2} / 2$ for some fixed positive definite inner product in $\mathfrak{g}^{*}$. Then there exists $k_{0}>0$ such that for all $k<k_{0}$ the hypersurface $\Sigma_{k}$ is stable.

Proof We will show that there exists $k_{0}>0$ such that for all $k<k_{0}$ the Euler vector field $E$ is geodesible in $\mathbf{S}_{k}$. By Lemma 2.3 this implies the stability of $\Sigma_{k}$.

Since $\sigma$ is symplectic, inspection of the bracket given in (ii) of Lemma 6.1 shows that for all $k$ sufficiently small, the Poisson bracket $\{,\}_{\sigma}$ is nondegenerate on the set $H^{-1}([0, k]) \subset \mathfrak{g}^{*}$. In other words, for all $\mu \in H^{-1}([0, k])$ the linear map $B_{\mu}: \mathfrak{g} \rightarrow \mathfrak{g}^{*}$ given by

$$
B_{\mu}(X)(w):=\mu([X, w])-\sigma_{e}(X, w)
$$

is invertible. Let $A: \mathfrak{g} \rightarrow \mathfrak{g}^{*}$ be $A(X)(w):=\sigma_{e}(X, w)$. We may write

$$
E(\mu)=E^{0}(\mu)-J \mu
$$

where $J: \mathfrak{g}^{*} \rightarrow \mathfrak{g}^{*}$ is defined by $J \mu(w):=\sigma\left(d_{\mu} H, w\right)$ and $E^{0}$ is the Euler vector field for $\sigma=0$. A short computation shows that $J$ is uniquely determined by

$$
\widehat{\sigma}(\xi, \eta):=\sigma\left(A^{-1} \xi, A^{-1} \eta\right)=\left\langle\xi, J^{-1} \eta\right\rangle .
$$

For $k$ small the Poisson structure $\{,\} \sigma$ is induced by the symplectic form $\varpi$ in $H^{-1}([0, k])$ given by

$$
\varpi(\mu)\left(B_{\mu}(v), B_{\mu}(w)\right):=\mu([v, w])-\sigma(v, w) .
$$

Inverting $B_{\mu}$ we get

$$
\varpi=-\hat{\sigma}+d \tau
$$

where $\tau$ is a 1 -form such that $|\tau(\mu)|=O(k)$ for $\mu \in H^{-1}([0, k])$. (The 2-form $\varpi+\hat{\sigma}$ is $O\left(k^{1 / 2}\right)$, so we can choose a primitive which is $O(k)$.) Let $\alpha$ be the 1 -form in $\mathfrak{g}^{*}$ given by $\alpha(\mu)(\eta):=\left\langle\mu, J^{-1} \eta\right\rangle / 2$. Then

$$
\varpi=d(-\alpha+\tau) \text {. }
$$

On $\mathbf{S}_{k}$ we have $i_{E} \varpi=0$ and

$$
\begin{aligned}
(-\alpha+\tau)(\mu)(E(\mu)) & =\alpha(\mu)(J \mu)-\alpha(\mu)\left(E^{0}(\mu)\right)+\tau(\mu)\left(E^{0}(\mu)\right)-\tau(\mu)(J \mu) \\
& =k+O\left(k^{3 / 2}\right),
\end{aligned}
$$

because $\alpha(J \mu)=|\mu|^{2} / 2=k$ and $E^{0}(\mu)$ is quadratic in $\mu$. Thus for $k$ small $(-\alpha+\tau)(E)>0$ on $\mathbf{S}_{k}$ and $E$ is geodesible (in fact contact). 
Suppose now that $G$ admits a cocompact lattice $\Gamma \subset G$. Then we have a symplectic manifold $(\Gamma \backslash G, \sigma)$. Any left invariant metric will give rise to a magnetic flow with stable low energy levels. We now look in detail at a concrete example.

Example 6.20 Consider the 4-dimensional nilpotent Lie algebra $\mathfrak{g}$ with basis $\left\{X_{1}\right.$, $\left.X_{2}, X_{3}, X_{4}\right\}$ whose only nonzero bracket is $\left[X_{1}, X_{2}\right]=X_{3}$. If $\left\{e_{1}, e_{2}, e_{3}, e_{4}\right\}$ is the dual basis of $\mathfrak{g}^{*}$, then $d e_{1}=d e_{2}=d e_{4}=0$ and $d e_{3}=-e_{1} \wedge e_{2}$. The symplectic 2 -form is

$$
\sigma=-e_{1} \wedge e_{3}-e_{2} \wedge e_{4}
$$

If $x_{i}$ denote the coordinates of $\mu \in \mathfrak{g}^{*}$ in the given basis we easily find that the nonzero Poisson brackets of $\{,\} \sigma$ are

$$
\left\{x_{1}, x_{2}\right\}=x_{3},\left\{x_{1}, x_{3}\right\}=\left\{x_{2}, x_{4}\right\}=1 .
$$

We see right away that $\{$,$\} is nondegenerate at every point \mu \in \mathfrak{g}^{*}$. In this case it is easy to invert the operator $B_{\mu}$ of the previous proposition and we find $\varpi$ to be

$$
\begin{aligned}
\varpi & =-\sigma-x_{3} d x_{3} \wedge d x_{4} \\
& =d x_{1} \wedge d x_{3}+d x_{2} \wedge d x_{4}-x_{3} d x_{3} \wedge d x_{4} .
\end{aligned}
$$

If we let

then

$$
\theta:=\frac{x_{1} d x_{3}-x_{3} d x_{1}+x_{2} d x_{4}-x_{4} d x_{2}}{2}-\frac{x_{3}^{2} d x_{4}}{2}
$$

$$
\varpi=d \theta \text {. }
$$

Now take $H(\mu)=|\mu|^{2} / 2$, where the inner product is defined so that $\left\{e_{1}, e_{2}, e_{3}, e_{4}\right\}$ is an orthonormal basis. The Euler vector field $E_{H}$ is easily computed using Lemma 6.1. One finds

$$
\begin{aligned}
E_{H}(\mu) & =\left(-x_{2} x_{3}-x_{3}, x_{1} x_{3}-x_{4}, x_{1}, x_{2}\right) \\
& =E_{H}^{0}(\mu)-J \mu \\
& =\left(-x_{2} x_{3}, x_{1} x_{3}, 0,0\right)-\left(x_{3}, x_{4},-x_{1},-x_{2}\right) .
\end{aligned}
$$

Next we compute $\theta\left(E_{H}\right)$ and we find

Using that

$$
\begin{gathered}
\theta\left(E_{H}\right)=\frac{x_{1}^{2}+x_{2}^{2}+x_{3}^{2}+x_{4}^{2}}{2}-\frac{x_{1} x_{3} x_{4}}{2} . \\
\frac{x_{1} x_{3} x_{4}}{2}=-\frac{d\left(x_{2} x_{4}\right)\left(E_{H}\right)}{2}+\frac{x_{4}^{2}-x_{2}^{2}}{2}
\end{gathered}
$$


we obtain

$$
\begin{gathered}
\left(\theta+\frac{d\left(x_{2} x_{4}\right)}{2}\right)\left(E_{H}\right)=\frac{x_{1}^{2}+2 x_{2}^{2}+x_{3}^{2}}{2} . \\
\varphi:=\theta+\frac{d\left(x_{2} x_{4}\right)}{2}
\end{gathered}
$$

Then

is a primitive of $\varpi$ such that on $\mathbf{S}_{k}$

$$
\varphi\left(E_{H}\right) \geq 0
$$

with equality if and only if $x_{1}=x_{2}=x_{3}=0$ and $x_{4}= \pm \sqrt{2 k}$. Since this set is not invariant under the flow of $E_{H}$ we conclude that for any invariant Borel probability measure $v$ on $\mathbf{S}_{k}$ we have

$$
\int_{\mathbf{S}_{k}} \varphi\left(E_{H}\right) d v>0
$$

and thus $E_{H}$ is in fact of contact type by Theorem 2.7.

Summarizing, we have shown that $E_{H}$ is geodesible on $\mathbf{S}_{k}$ for any $k>0$. Let $G$ be the simply connected Lie group with Lie algebra $\mathfrak{g}$. This group certainly admits cocompact lattices $\Gamma$. The manifold $(\Gamma \backslash G, \sigma)$ is symplectic (with first Betti number 3 in fact) and with the left invariant metric considered above we obtain that $\Sigma_{k}$ is stable for any $k>0$. Every compact set here is displaceable by [10, Theorem B] and the Mañé critical value is $\infty$ since $\Gamma$ is nilpotent.

A realisation of $G$ is $\mathcal{H} \times \mathbb{R}$ where $\mathcal{H}$ is the 3-dimensional Heisenberg group. The metric is just the product metric. It is easy to see that $\mathcal{H}$ has no contractible closed geodesics, hence the same is true for $G$.

\section{Proofs of the theorems}

\subsection{Proof of Theorem 1.1}

For the case of tame stable hypersurfaces and tame stable homotopies the theorem is a consequence of Theorem 4.14 and Theorem 4.15. Note that we could take as definition of $\operatorname{RFH}(\Sigma)$ either $\overline{R F H}(\Sigma, V)$ or $\underline{R H H}(\Sigma, V)$. The claims for the virtually contact case are straightforward extensions of the contact case treated in [13]. Indeed, the virtually contact condition implies that the period-action inequality for almost Reeb orbits of Proposition 3.2 in [13] continues to hold with constants now depending on the constants appearing in the virtually contact condition. Here we use that $\pi_{1}(\Sigma)$ injects into $\pi_{1}(M)$. Similarly, for the time-dependent case Proposition 3.4 in [13] continues to hold for a virtually contact homotopy. Having established these two Propositions the forthcoming arguments can be repeated just word-by-word. 
Remark In the virtually contact case, one can replace the condition that $\pi_{1}(\Sigma)$ injects into $\pi_{1}(V)$ by a weaker condition which we now explain.

Consider a Hamiltonian structure $\omega$ on a closed odd dimensional manifold $\Sigma$. We call the pair $(\Sigma, \omega)$ virtually contact, if on a cover $\pi: \widetilde{\Sigma} \rightarrow \Sigma$ there exists a primitive $\lambda \in \Omega^{1}(\widetilde{\Sigma})$ of $\pi^{*} \omega$ with the property that for one and hence every Riemannian metric $g$ on $\Sigma$ there exists a constant $c=c(g)>0$ such that

$$
\|\lambda\|_{\pi^{*} g} \leq c, \quad\|\xi\|_{\pi^{*} g} \leq c|\lambda(\xi)|, \quad \forall \xi \in \operatorname{ker}\left(\pi^{*} \omega\right)
$$

We refer to the one-form $\lambda$ as a bounded primitive. A closed hypersurface $\Sigma$ in a symplectic manifold $(V, \omega)$ is called of virtual restricted contact type, if there exists a cover $\pi: \tilde{V} \rightarrow V$ and a primitive $\lambda$ of $\pi^{*} \omega$ such that $\left.\lambda\right|_{\pi^{-1}(\Sigma)}$ is a bounded primitive for $\left(\Sigma,\left.\omega\right|_{\Sigma}\right)$.

Lemma 7.1 Assume that $(V, \omega)$ is symplectically aspherical, ie $\omega$ vanishes on $\pi_{2}(V)$, and $\Sigma \subset V$ is a closed hypersurface with the property that $\left(\Sigma,\left.\omega\right|_{\Sigma}\right)$ is virtually contact and the inclusion homomorphism $i_{*}: \pi_{1}(\Sigma) \rightarrow \pi_{1}(V)$ is injective. Then $\Sigma$ is of virtual restricted contact type.

Proof Let $\pi: \tilde{V} \rightarrow V$ be the universal cover of $V$. Since $\tilde{V}$ is simply connected we have by Hurewicz theorem that

$$
H_{2}(\tilde{V} ; \mathbb{Z})=\pi_{2}(\tilde{V})=\pi_{2}(V) .
$$

Since $(V, \omega)$ is symplectically aspherical we deduce that $\pi^{*} \omega$ vanishes on $H_{2}(\tilde{V} ; \mathbb{Z})$ and hence

$$
\left[\pi^{*} \omega\right]=0 \in H_{d R}^{2}(\tilde{V}) .
$$

We conclude that there exists $\lambda_{0} \in \Omega^{1}(\tilde{V})$ such that

$$
d \lambda_{0}=\pi^{*} \omega .
$$

Because $\left(\Sigma,\left.\omega\right|_{\Sigma}\right)$ is virtually contact, there exists a bounded primitive $\lambda_{1}$ on a cover of $\Sigma$. Since $\lambda_{1}$ is bounded, there exists a bounded lift $\tilde{\lambda}_{1}$ to $\pi^{-1}(\Sigma) \subset \widetilde{V}$. Note that

$$
\left.d \lambda_{0}\right|_{\pi^{-1}(\Sigma)}-d \tilde{\lambda}_{1}=\left.\pi^{*} \omega\right|_{\pi^{-1}(\Sigma)}-\left.\pi^{*} \omega\right|_{\pi^{-1}(\Sigma)}=0 .
$$

We conclude that $\left.\lambda_{0}\right|_{\pi^{-1}(\Sigma)}-\tilde{\lambda}_{1}$ defines a class

$$
\left[\left.\lambda_{0}\right|_{\pi^{-1}(\Sigma)}-\tilde{\lambda}_{1}\right] \in H_{d R}^{1}\left(\pi^{-1}(\Sigma)\right) .
$$

However, since $i_{*}: \pi_{1}(\Sigma) \rightarrow \pi_{1}(V)$ is injective, each connected component of $\pi^{-1}(\Sigma)$ is simply connected. Therefore $H_{d R}^{1}\left(\pi^{-1}(\Sigma)\right)=\{0\}$ and consequently there exists 
$f \in C^{\infty}\left(\pi^{-1}(\Sigma), \mathbb{R}\right)$ such that

$$
d f=\left.\lambda_{0}\right|_{\pi^{-1}(\Sigma)}-\tilde{\lambda}_{1} .
$$

Extend $f$ to a smooth map $\tilde{f} \in C^{\infty}(\tilde{V}, \mathbb{R})$ and set

$$
\begin{gathered}
\lambda=\lambda_{0}-d \tilde{f} . \\
\left.\lambda\right|_{\pi^{-1}(\Sigma)}=\left.\lambda_{0}\right|_{\pi^{-1}(\Sigma)}-d f=\tilde{\lambda}_{1}
\end{gathered}
$$

Noting that

we conclude that $\lambda$ is a primitive of $\pi^{*} \omega$ whose restriction to $\pi^{-1}(\Sigma)$ is bounded. This finishes the proof of the lemma.

Theorem 1.1 continues to hold with the same proof if we assume that $\Sigma$ is of virtual restricted contact type. The hypersurfaces of Lemma 5.1 are of virtual restricted contact type, but when $\operatorname{dim} M=2, \pi_{1}(\Sigma)$ does not inject into $\pi_{1}\left(T^{*} M\right)$.

\subsection{Proof of Theorem 1.2}

Let $\sigma=0$. By Lemma 5.2, the Mañé critical value is given by $c=\max U$ and all regular level sets $\Sigma_{k}$ are of restricted contact type, so $\operatorname{RFH}\left(\Sigma_{k}\right)$ is defined.

For $k>c$ each $\Sigma_{k}$ is regular. By the invariance of Rabinowitz Floer homology under contact homotopies, we can compute $\operatorname{RFH}\left(\Sigma_{k}\right)$ for zero potential. In this case it is nonzero by Corollary 1.12 in [14]. In particular, $\Sigma_{k}$ is nondisplaceable for $k \geq c$. The dynamics on $\Sigma_{k}, k>c$, is given by the geodesic flow of the Jacobi metric $g /(k-U)$. The level set $\Sigma_{c}$ is singular.

For $k<c, \Sigma_{k}$ is displaceable because it misses one fibre.

\subsection{Proof of Theorem 1.3}

For $k>c, \Sigma_{k}$ is virtually contact by Lemma 5.1 (in fact it is of virtual restricted contact type) thus $\operatorname{RFH}\left(\Sigma_{k}\right)$ is defined and invariant under virtually contact homotopies. In particular the isomorphism type of $\operatorname{RFH}\left(\Sigma_{k}\right)$ is independent of $k$.

Consider a path of Riemannian metrics $[0,1] \ni t \mapsto g_{t}$ such that $g_{0}=g$ and $g_{1}$ is a metric of negative curvature. By taking $k>\max _{t \in[0,1]} c_{t}$ and using that the isomorphism type of $\operatorname{RFH}\left(\Sigma_{k, t}\right)$ is independent of $t$ as well, we may suppose that $g$ itself is negatively curved. But it is well known that the geodesic flow of a negatively curved metric is Anosov and does not have nontrivial closed contractible orbits. By structural stability the same is true for the orbits of $\Sigma_{k}$ provided $k$ is sufficiently large. It follows from Theorem 1.1 that $\operatorname{RFH}\left(\Sigma_{k}\right)$ does not vanish and thus $\Sigma_{k}$ is nondisplaceable. 


\subsection{Proof of Theorem 1.4}

This is just Theorem 5.6.

\subsection{Proof of Theorem 1.5}

We argue by contradiction. Suppose the energy level $\Sigma_{c}$ is stable. We will show that $\Sigma_{c}$ must contain a contractible periodic orbit. By Lemma 2.5 there exists a tubular neighborhood $(-\varepsilon, \varepsilon) \times \Sigma_{c}$ of $\Sigma_{c}$ such that the characteristic foliations on $\{r\} \times \Sigma_{c}$ are conjugate for all $r \in(-\varepsilon, \varepsilon)$. But by hypothesis, every compact set contained in the set $H^{-1}(-\infty, c)$ is displaceable, hence we may apply the main result of Schlenk [64], to conclude that for almost every $r$, with $r<0,\{r\} \times \Sigma_{c}$ carries a closed contractible orbit. By stability, $\Sigma_{c}$ must also carry a closed contractible orbit.

\subsection{Proofs of Theorems 1.6, 1.8 and 1.9}

Theorem 1.6 follows from the lemmas in Section 6.2 and Theorem 1.1. Theorem 1.8 follows from the lemmas in Section 6.3 and Theorem 1.1. Theorem 1.9 follows from the results in Section 6.1 and Theorem 1.1.

\section{References}

[1] A Abbondandolo, M Schwarz, Estimates and computations in Rabinowitz-Floer homology, J. Topol. Anal. 1 (2009) 307-405 MR2597650

[2] R Abraham, J E Marsden, Foundations of mechanics, Benjamin/Cummings, Reading, MA (1978) MR515141 Second edition, revised and enlarged, With the assistance of T Raţiu and R Cushman

[3] D V Anosov, J G Sinaй, Some smooth ergodic systems, Uspehi Mat. Nauk 22 (1967) 107-172 MR0224771

[4] V I Arnol'd, On some problems in symplectic topology, from: "Topology and geometry—Rohlin Seminar", (O Y Viro, editor), Lecture Notes in Math. 1346, Springer, Berlin (1988) 1-5 MR970068

[5] V I Arnol'd, Mathematical methods of classical mechanics, second edition, Graduate Texts in Math. 60, Springer, New York (1989) MR997295 Translated from the Russian by K Vogtmann and A Weinstein

[6] M Audin, F Lalonde, L Polterovich, Symplectic rigidity: Lagrangian submanifolds, from: "Holomorphic curves in symplectic geometry", (M Audin, J Lafontaine, editors), Progr. Math. 117, Birkhäuser, Basel (1994) 271-321 MR1274934

[7] P Bernard, Symplectic aspects of Mather theory, Duke Math. J. 136 (2007) 401-420 MR2309170 
[8] K Burns, GP Paternain, Anosov magnetic flows, critical values and topological entropy, Nonlinearity 15 (2002) 281-314 MR1888853

[9] L T Butler, An optical Hamiltonian and obstructions to integrability, Nonlinearity 19 (2006) 2123-2135 MR2256655

[10] L T Butler, G P Paternain, Magnetic flows on Sol-manifolds: dynamical and symplectic aspects, Comm. Math. Phys. 284 (2008) 187-202 MR2443302

[11] C Camacho, A Lins Neto, Geometric theory of foliations, Birkhäuser, Boston (1985) MR824240 Translated from the Portuguese by S E Goodman

[12] K Cieliebak, U A Frauenfelder, Morse homology on noncompact manifolds arXiv: 0911.1805

[13] K Cieliebak, U A Frauenfelder, A Floer homology for exact contact embeddings, Pacific J. Math. 239 (2009) 251-316 MR2461235

[14] K Cieliebak, U A Frauenfelder, A Oancea, Rabinowitz Floer homology and symplectic homology, to appear in Ann. Sci. École Norm. Sup. arXiv:0903.0768

[15] K Cieliebak, UA Frauenfelder, GP Paternain, Stability is not open arXiv: 0908.2540

[16] K Cieliebak, K Mohnke, Compactness for punctured holomorphic curves, J. Symplectic Geom. 3 (2005) 589-654 MR2235856

[17] K Cieliebak, E Volkov, First steps in stable Hamiltonian topology arXiv: 1003.5084

[18] G Contreras, The Palais-Smale condition on contact type energy levels for convex Lagrangian systems, Calc. Var. Partial Differential Equations 27 (2006) 321-395 MR2260806

[19] G Contreras, J Delgado, R Iturriaga, Lagrangian flows: the dynamics of globally minimizing orbits. II, Bol. Soc. Brasil. Mat. (N.S.) 28 (1997) 155-196 MR1479500

[20] G Contreras, R Iturriaga, G P Paternain, M Paternain, Lagrangian graphs, minimizing measures and Mañé's critical values, Geom. Funct. Anal. 8 (1998) 788-809 MR1650090

[21] G Contreras, L Macarini, G P Paternain, Periodic orbits for exact magnetic flows on surfaces, Int. Math. Res. Not. (2004) 361-387 MR2036336

[22] N S Dairbekov, G P Paternain, On the cohomological equation of magnetic flows, Mat. Contemp. 34 (2008) 155-193 MR2588611

[23] Y Eliashberg, A Givental, H Hofer, Introduction to symplectic field theory, from: "GAFA 2000 (Tel Aviv, 1999)", (N Alon, J Bourgain, A Connes, M Gromov, V Milman, editors), Geom. Funct. Anal. Special Volume, Part II (2000) 560-673 MR1826267

[24] A Fathi, E Maderna, Weak KAM theorem on non compact manifolds, Nonlinear Differential Equations Appl. 14 (2007) 1-27 MR2346451 
[25] R Feres, Geodesic flows on manifolds of negative curvature with smooth horospheric foliations, Ergodic Theory Dynam. Systems 11 (1991) 653-686 MR1145615

[26] R Feres, A Katok, Invariant tensor fields of dynamical systems with pinched Lyapunov exponents and rigidity of geodesic flows, Ergodic Theory Dynam. Systems 9 (1989) 427-432 MR1016661

[27] P Foulon, Feuilletages des sphères et dynamiques Nord-Sud, C. R. Acad. Sci. Paris Sér. I Math. 318 (1994) 1041-1042 MR1281888

[28] H Furstenberg, The unique ergodicity of the horocycle flow, from: "Recent advances in topological dynamics (Proc. Conf., Yale Univ., New Haven, Conn., 1972; in honor of Gustav Arnold Hedlund)", (A Beck, editor), Lecture Notes in Math. 318, Springer, Berlin (1973) 95-115 MR0393339

[29] V L Ginzburg, On closed trajectories of a charge in a magnetic field. An application of symplectic geometry, from: "Contact and symplectic geometry (Cambridge, 1994)", (C B Thomas, editor), Publ. Newton Inst. 8, Cambridge Univ. Press (1996) 131-148 MR1432462

[30] V L Ginzburg, B Z Gürel, Periodic orbits of twisted geodesic flows and the WeinsteinMoser theorem, Comment. Math. Helv. 84 (2009) 865-907 MR2534483

[31] V L Ginzburg, E Kerman, Periodic orbits in magnetic fields in dimensions greater than two, from: "Geometry and topology in dynamics (Winston-Salem, NC, 1998/San Antonio, TX, 1999)”, (M Barge, K Kuperberg, editors), Contemp. Math. 246, Amer. Math. Soc. (1999) 113-121 MR1732375

[32] M Gromov, Pseudoholomorphic curves in symplectic manifolds, Invent. Math. 82 (1985) 307-347 MR809718

[33] M Gromov, Kähler hyperbolicity and $L_{2}$-Hodge theory, J. Differential Geom. 33 (1991) 263-292 MR1085144

[34] U Hamenstädt, Invariant two-forms for geodesic flows, Math. Ann. 301 (1995) 677698 MR1326763

[35] B Hasselblatt, Horospheric foliations and relative pinching, J. Differential Geom. 39 (1994) 57-63 MR1258914

[36] B Hasselblatt, Regularity of the Anosov splitting and of horospheric foliations, Ergodic Theory Dynam. Systems 14 (1994) 645-666 MR1304137

[37] M W Hirsch, C C Pugh, M Shub, Invariant manifolds, Lecture Notes in Math. 583, Springer, Berlin (1977) MR0501173

[38] H Hofer, K Wysocki, E Zehnder, A general Fredholm theory. I. A splicing-based differential geometry, J. Eur. Math. Soc. (JEMS) 9 (2007) 841-876 MR2341834

[39] H Hofer, K Wysocki, E Zehnder, A general Fredholm theory. II. Implicit function theorems, Geom. Funct. Anal. 19 (2009) 206-293 MR2507223

[40] H Hofer, E Zehnder, Symplectic invariants and Hamiltonian dynamics, Birkhäuser Advanced Texts: Basler Lehrbücher, Birkhäuser Verlag, Basel (1994) MR1306732 
[41] M Kanai, Differential-geometric studies on dynamics of geodesic and frame flows, Japan. J. Math. (N.S.) 19 (1993) 1-30 MR1231509

[42] A Katok, B Hasselblatt, Introduction to the modern theory of dynamical systems, Ency. of Math. and its Appl. 54, Cambridge Univ. Press (1995) MR1326374 With a supplementary chapter by Katok and L Mendoza

[43] W Klingenberg, Riemannian geometry, de Gruyter Studies in Math. 1, de Gruyter, Berlin (1982) MR666697

[44] G Knieper, Hyperbolic dynamics and Riemannian geometry, from: "Handbook of dynamical systems, Vol. 1A", (B Hasselblatt, A Katok, editors), North-Holland, Amsterdam (2002) 453-545 MR1928523

[45] F Laudenbach, J-C Sikorav, Hamiltonian disjunction and limits of Lagrangian submanifolds, Internat. Math. Res. Notices (1994) 161 ff., approx. 8 pp. MR1266111

[46] G Lu, The Weinstein conjecture on some symplectic manifolds containing the holomorphic spheres, Kyushu J. Math. 52 (1998) 331-351 MR1645455

[47] S Mac Lane, Categories for the working mathematician, second edition, Graduate Texts in Math. 5, Springer, New York (1998) MR1712872

[48] L Macarini, G P Paternain, On the stability of Mañé critical hypersurfaces, to appear in Calc. Var. Partial Diff. Equations arXiv:0910.5728

[49] R Mañé, Lagrangian flows: the dynamics of globally minimizing orbits, Bol. Soc. Brasil. Mat. (N.S.) 28 (1997) 141-153 MR1479499

[50] J N Mather, Action minimizing invariant measures for positive definite Lagrangian systems, Math. Z. 207 (1991) 169-207 MR1109661

[51] D McDuff, Applications of convex integration to symplectic and contact geometry, Ann. Inst. Fourier (Grenoble) 37 (1987) 107-133 MR894563

[52] D McDuff, D Salamon, Introduction to symplectic topology, second edition, Oxford Math. Monogr., The Clarendon Press, Oxford Univ. Press, New York (1998) MR1698616

[53] D McDuff, D Salamon, $J$-holomorphic curves and symplectic topology, Amer. Math. Soc. Coll. Publ. 52, Amer. Math. Soc. (2004) MR2045629

[54] W Merry, Closed orbits of a charge in a weakly exact magnetic field, to appear in Pacific J. Math. arXiv:0906.1192

[55] W Merry, On the Rabinowitz Floer homology of twisted cotangent bundles arXiv: 1002.0162

[56] W Merry, GP Paternain, Stability of Anosov Hamiltonian structures arXiv: 0903.3969

[57] K Nomizu, On the cohomology of compact homogeneous spaces of nilpotent Lie groups, Ann. of Math. (2) 59 (1954) 531-538 MR0064057 
[58] S P Novikov, The Hamiltonian formalism and a multivalued analogue of Morse theory, Uspekhi Mat. Nauk 37 (1982) 3-49, 248 MR676612

[59] W Parry, Synchronisation of canonical measures for hyperbolic attractors, Comm. Math. Phys. 106 (1986) 267-275 MR855312

[60] G P Paternain, Magnetic rigidity of horocycle flows, Pacific J. Math. 225 (2006) 301323 MR2233738

[61] G P Paternain, M Paternain, Critical values of autonomous Lagrangian systems, Comment. Math. Helv. 72 (1997) 481-499 MR1476061

[62] G P Paternain, L Polterovich, K F Siburg, Boundary rigidity for Lagrangian submanifolds, non-removable intersections, and Aubry-Mather theory, Mosc. Math. J. 3 (2003) 593-619, 745 MR2025275 Dedicated to Vladimir I Arnold on the occasion of his 65 th birthday

[63] L Polterovich, An obstacle to non-Lagrangian intersections, from: "The Floer memorial volume”, (A Weinstein, E Zehnder, editors), Progr. Math. 133, Birkhäuser, Basel (1995) 575-586 MR1362842

[64] F Schlenk, Applications of Hofer's geometry to Hamiltonian dynamics, Comment. Math. Helv. 81 (2006) 105-121 MR2208800

[65] P Scott, The geometries of 3-manifolds, Bull. London Math. Soc. 15 (1983) 401-487 MR705527

[66] D Sullivan, A foliation of geodesics is characterized by having no "tangent homologies”, J. Pure Appl. Algebra 13 (1978) 101-104 MR508734

[67] C Viterbo, Symplectic homogeneization arXiv:0801.0206

[68] C Viterbo, Functors and computations in Floer homology with applications. I, Geom. Funct. Anal. 9 (1999) 985-1033 MR1726235

[69] A W Wadsley, Geodesic foliations by circles, J. Differential Geometry 10 (1975) 541549 MR0400257

[70] A Weinstein, On the hypotheses of Rabinowitz' periodic orbit theorems, J. Differential Equations 33 (1979) 353-358 MR543704

Mathematisches Institut, Ludwig-Maximilians-Universität München

Theresienstr 39, 80333 München, Germany

Department of Mathematics and Research Institute of Mathematics, Seoul National University San56-1 Shinrim-dong Kwanak-gu, Seoul 151-747, Korea

Department of Pure Mathematics and Mathematical Statistics, University of Cambridge Wilberforce Road, Cambridge CB3 OWB, United Kingdom

kai@math.lmu.de, frauenf@snu.ac.kr, g.p.paternain@dpmms.cam.ac.uk

Proposed: Leonid Polterovich

Seconded: Danny Calegari, Yasha Eliashberg
Received: 3 November 2009

Revised: 25 April 2010 